\title{
Twisted elliptic multiple zeta values and non-planar one-loop open-string amplitudes
}

\author{
Johannes Broedel $^{\mathrm{a}}$, Nils Matthes ${ }^{\mathrm{b}}$, Gregor Richter ${ }^{\mathrm{a}, \mathrm{c}}$, Oliver Schlotterer ${ }^{\mathrm{c}}$ \\ ${ }^{a}$ Institut für Mathematik und Institut für Physik, \\ Humboldt-Universität zu Berlin \\ IRIS Adlershof, Zum Großen Windkanal 6, 12489 Berlin, Germany \\ ${ }^{b}$ Max-Planck-Institut für Mathematik, \\ Vivatsgasse 7, 53111 Bonn, Germany \\ ${ }^{c}$ Max-Planck-Institut für Gravitationsphysik, \\ Albert-Einstein-Institut \\ Am Mühlenberg 1, 14476 Potsdam, Germany \\ jbroedel@physik.hu-berlin.de, nilsmath@mpim-bonn.mpg.de, \\ grichter@physik.hu-berlin.de, olivers@aei.mpg.de
}

\begin{abstract}
We consider a generalization of elliptic multiple zeta values, which we call twisted elliptic multiple zeta values. These arise as iterated integrals on an elliptic curve from which a rational lattice has been removed. At the cusp, twisted elliptic multiple zeta values are shown to degenerate to cyclotomic multiple zeta values in the same way as elliptic multiple zeta values degenerate to classical multiple zeta values. We investigate properties of twisted elliptic multiple zeta values and utilize them in the evaluation of the non-planar part of the four-point one-loop open- superstring amplitude.
\end{abstract}




\section{Contents}

1 Introduction $\quad 3$

2 From elliptic to twisted elliptic multiple zeta values 5



2.2 General properties of elliptic iterated integrals and (t)eMZVs $\ldots \ldots \ldots \ldots$

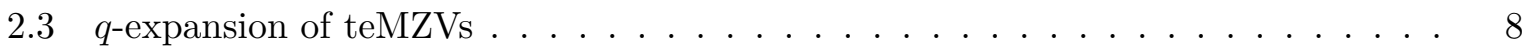

3 q-expansion for twisted elliptic multiple zeta values $\quad 10$

3.1 Differential equation . . . . . . . . . . . . . . . . . . . 10

3.2 Constant terms for generic twists . . . . . . . . . . . . . . . 12

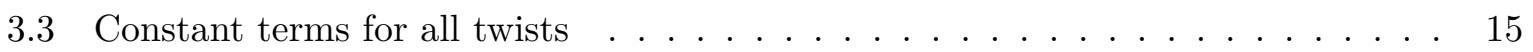

4 One-loop open-string amplitude $\quad 17$

4.1 The four-point integrals . . . . . . . . . . . . . . . . . . . . . 19

4.2 The genus-one Green function as an elliptic iterated integral . . . . . . . . . . 21

4.3 Non-planar contribution to the four-point amplitude . . . . . . . . . . . . 24

5 Conclusions $\quad 29$

$\begin{array}{ll}\text { A Jacobi theta functions } & 30\end{array}$

$\begin{array}{ll}\text { B Weighting functions } & 30\end{array}$

C A teMZV with proper rational twist $b=1 / 2 \quad 31$

D MZVs and cyclotomic MZVs

E Details on the differential equation of teMZVs $\quad 34$

\begin{tabular}{ll|l} 
F & Differential equation for proper rational twists & 37
\end{tabular}

G Properties of teMZVs $\quad 39$

G.1 Constant terms for generic twists . . . . . . . . . . . . . . . . . . . 39

G.2 Constant terms for proper rational twists $\ldots \ldots \ldots \ldots \ldots$

G.3 teMZV relations for the string amplitude . . . . . . . . . . . . . . 40

G.4 q-expansions for the string amplitude . . . . . . . . . . . 41

H The non-planar integral along with $\operatorname{Tr}\left(t^{1} t^{2} t^{3}\right) \operatorname{Tr}\left(t^{4}\right)$

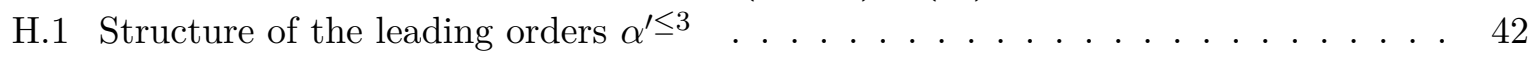



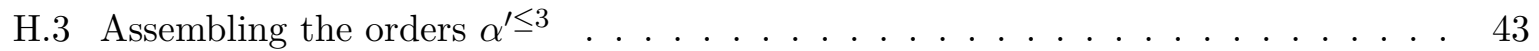

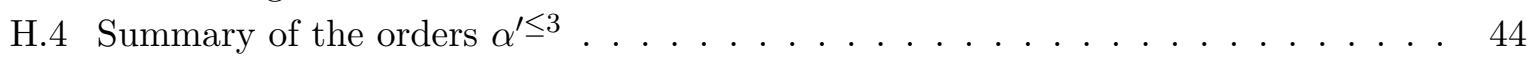

I Sample integrals in non-planar string amplitudes $\quad 44$

I.1 The $d_{11}^{3}$ integral from $P_{i j} Q_{i k} Q_{j k} \ldots \ldots \ldots \ldots \ldots \ldots \ldots \ldots$

I.2 The $e_{4}^{3}$ integral from $P_{i j} P_{i k} P_{j k} \ldots \ldots \ldots \ldots \ldots \ldots \ldots \ldots \ldots \ldots \ldots$

J Some all-order contributions 46 


\section{Introduction}

Within the program of studying iterated integrals on Riemann surfaces of various genera, the genus-zero case, which leads to multiple zeta values (MZVs) [1-3], is the starting point and takes the most prominent rôle. During the last years, however, the genus-one situation has received more attention: various iterated integrals on the elliptic curve as well as associated periods and elliptic associators have been investigated $[4-7]$.

The simplest genus-one generalizations of MZVs are elliptic multiple zeta values (eMZVs), which arise from iterated integrals on the once-punctured elliptic curve, that is the elliptic curve where the origin is removed [8. In this article, the notion of eMZVs is extended to twisted elliptic multiple zeta values (teMZVs), which are iterated integrals on a multiply-punctured elliptic curve. While for eMZVs it is sufficient to remove the origin, teMZVs arise when a lattice with rational coordinates as visualized in figure 1 is removed from the elliptic curve.

The iterated integrals to be considered are performed over a path parallel to the real axis and are therefore a generalization of Enriquez' $A$-cycle eMZVs. If no lattice is removed, we obtain A-cycle eMZVs by definition. ${ }^{1}$ A slight technical difficulty, which was absent for eMZVs, is that the integrands giving rise to teMZVs might have additional poles along the path of integration. We address the problem by suggesting a rather natural regularization scheme, which essentially amounts to integrating over an infinitesimal deformation of the real axis.

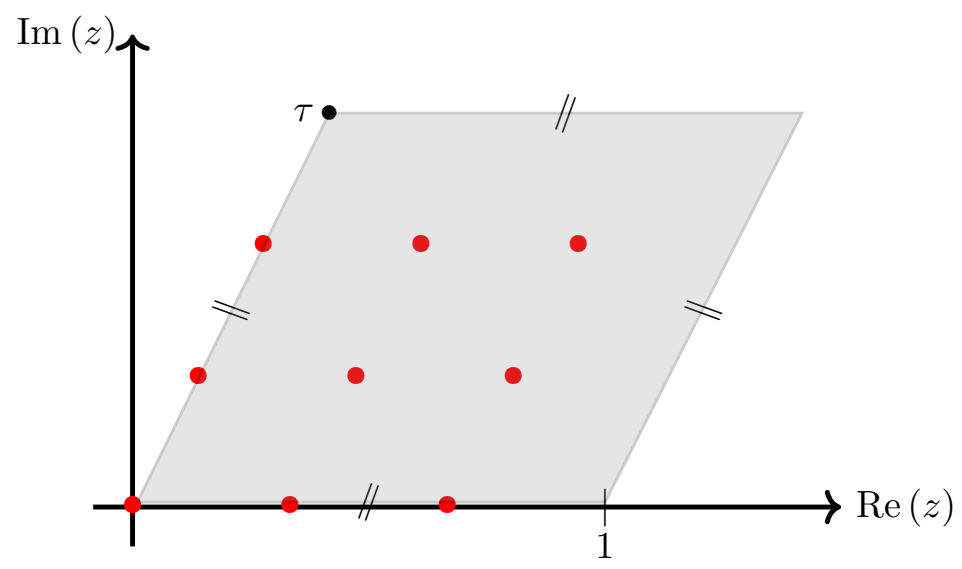

Figure 1: The shaded region represents the elliptic curve $\mathbb{C} /(\mathbb{Z}+\mathbb{Z} \tau)$, where edges marked by $/ /$ and $\approx$ are identified. In the setup of teMZVs, points from the lattice $\mathbb{Q}+\mathbb{Q} \tau$ are removed from the elliptic curve. Here we show the example $\left\{0, \frac{1}{3}, \frac{2}{3}\right\}+\left\{0, \frac{1}{3}, \frac{2}{3}\right\} \tau$.

A crucial tool in the study of eMZVs was the existence of a certain first-order linear differential equation, expressing eMZVs as special linear combinations of iterated integrals of Eisenstein series and MZVs $[7,8,10,11]$. In particular, this is an efficient way to compute their $q$-expansion, which is instrumental for finding (as well as excluding) linear relations among eMZVs. Even more so, since iterated integrals of Eisenstein series are linearly independent [12], the differential equation amounts to a decomposition of eMZVs into basic constituents, which reduces the study of relations among eMZVs to solving linear systems of equations. One of the main results of this article is the generalization of this differential equation to teMZVs. We find that the occurrence of the classical Eisenstein series (for $\mathrm{SL}_{2}(\mathbb{Z})$ ) in the differential equation for eMZVs naturally generalizes to an occurrence of certain weighting functions $f^{(n)}(s+r \tau, \tau)$, where $r, s \in \mathbb{Q}$, and

\footnotetext{
${ }^{1}$ It is worth noting that there is no structural problem in defining twisted $B$-cycle eMZVs by considering integration paths parallel to the direction of the modular parameter $\tau 9$.
} 
the latter are also known to be modular forms for congruence subgroups of $\mathrm{SL}_{2}(\mathbb{Z})$. Likewise, one can again identify a procedure delivering the boundary data for teMZVs at the cusp $i \infty$ of the modular parameter $\tau$, and relating them to integrals over genus-zero Riemann surfaces in a natural way. While for eMZVs this procedure leads to MZVs, in the case of teMZVs we obtain cyclotomic MZVs 3,13 15. A further parallel to eMZVs is the existence of shuffle and Fay relations 10,16 .

Scattering amplitudes in open-superstring theories have been recently noticed as a rewarding setup where iterated integrals on Riemann surfaces appear naturally. Generalizing the ubiquity of MZVs in tree-level amplitudes ${ }^{2}$, one-loop scattering amplitudes (corresponding to genus-one surfaces) provide a natural testing ground for eMZVs [30]. However, the analysis in ref. [30] was focused on the planar sector of the one-loop amplitude where the integrations are performed over a single boundary of a genus-one surface with cylinder topology.

In this article, teMZVs will be identified as the suitable language for the calculation of the non-planar part of the open-string one-loop amplitude: the extension of the iterated integrals to both boundaries of the cylinder leads to the class of teMZVs with twist $\tau / 2$. We will employ these teMZVs to calculate non-planar contributions to the low-energy expansion ${ }^{3}$ of the fourpoint one-loop open-string amplitude. Explicit results will be given up to the third subleading low-energy order which are checked to match the expressions available in the literature at the first subleading low-energy order 31$]$ and at the cusp $[32$. As in the planar case, their efficient computation crucially relies on the differential equation satisfied by teMZVs.

Interestingly, our final result for the non-planar part of string scattering amplitudes in the cases considered can be expressed in terms of eMZVs alone, i.e. teMZVs with twist $\tau / 2$ cancel out. This observation relies on providing explicit formulas for certain linear combinations of teMZVs with twist $\tau / 2$ in terms of eMZVs, which can in turn be checked using their differential equation. We will provide physics arguments bolstering the conjecture that this feature will persist to all orders in the low-energy expansion, and it would be very interesting to find a mathematical explanation for this effect.

Finally, we expect the teMZVs defined here to be closely related to the monodromy of the universal twisted elliptic KZB equation to be studied in upcoming work of Calaque and Gonzalez 9. A particularly important aspect of their work is the definition of a twisted version of the derivation algebra, the untwisted version of which $[33,5,34,35]$ already appeared in the study of eMZVs $10,36,11$. Similar to the situation for eMZVs, this twisted derivation algebra might be capable of encoding the number of indecomposable teMZVs of a given weight and length.

In section 2 we introduce teMZVs, and discuss the expansion of their constituents with respect to the modular parameter of the elliptic curve. Thereby we set the stage for section 3 , where a differential equation for teMZVs w.r.t. $\tau$ as well as a procedure to extract their $\tau \rightarrow i \infty$ limit is presented. In section 4, the formalism is applied to the calculation of the non-planar contribution to the open-string one-loop scattering amplitude and the rôle of teMZVs therein is discussed. After concluding and pointing out a couple of open problems in section 5 we provide various appendices containing collections of definitions for the numerous objects appearing as well as several detailed calculations omitted in the main text.

\footnotetext{
${ }^{2}$ See $17-19$ for a discussion of the contributing iterated integrals on a genus-zero surface in the mathematics literature and 20,21] for a treatment via polylogarithms in a physics context. Moreover, the expansion of $n$-point disk integrals has been addressed via motivic MZVs $[22$ and the Drinfeld associator 23 (also see 24]). As a complementary approach, the relation of disk integrals to hypergeometric functions has been used to obtain $(n \leq 5)$-point expansions 25,26 and certain ranges of low-energy orders at $n \leq 7$ points, see e.g. 27 29.

${ }^{3}$ The low-energy expansion of string amplitudes refers to an expansion in the inverse string tension $\alpha^{\prime}$.
} 


\section{From elliptic to twisted elliptic multiple zeta values}

Elliptic multiple zeta values can be represented as iterated integrals on the multiply punctured elliptic curve $\mathbb{C} /(\mathbb{Z}+\mathbb{Z} \tau) \backslash\left\{b_{1}, \ldots, b_{\ell}\right\}$ with parameter $\tau$ in the upper half plane $\mathbb{H}$, where we denote $q=\exp (2 \pi i \tau)$. Starting from $\Gamma(; z)=1$, elliptic iterated integrals are defined recursively via

$$
\Gamma\left(\begin{array}{cccc}
n_{1} & n_{2} & \ldots & n_{\ell} \\
b_{1} & b_{2} & \ldots & b_{\ell}
\end{array} ; z\right)=\int_{0}^{z} \mathrm{~d} t f^{\left(n_{1}\right)}\left(t-b_{1}\right) \Gamma\left(\begin{array}{cccc}
n_{2} & \ldots & n_{\ell} \\
b_{2} & \ldots & b_{\ell}
\end{array} ; t\right), \quad z \in[0,1]
$$

where the interval of integration is $[0, z]$. As will be discussed in subsection 2.1, regularization prescriptions have to be specified, if $n_{i}=1$ and $b_{i} \in[0, z]$.

The weighting functions $f^{(n)}(z, \tau)$ arise as expansion coefficients of the doubly-periodic completion of the Eisenstein-Kronecker series, starting with

$$
f^{(0)}(z, \tau)=1, \quad f^{(1)}(z, \tau)=\frac{\theta_{1}^{\prime}(z, \tau)}{\theta_{1}(z, \tau)}+2 \pi i \frac{\operatorname{Im}(z)}{\operatorname{Im}(\tau)},
$$

see appendix B for details and conventions. They are doubly-periodic functions of alternating parity

$$
f^{(n)}(z+1, \tau)=f^{(n)}(z+\tau, \tau)=f^{(n)}(z, \tau), \quad f^{(n)}(-z, \tau)=(-1)^{n} f^{(n)}(z, \tau),
$$

and the function $f^{(1)}\left(z-b_{i}, \tau\right)$ in eq. $(2.2)$ acquires a pole at $z=b_{i}$ which requires regularization of eq. (2.1). Throughout the article, we will frequently omit noting the $\tau$-dependence of both weighting functions $f^{(n)}$ and elliptic iterated integrals eq. (2.1).

In refs. [30, 10], the main focus was on elliptic multiple zeta values, whose shifting parameters $b_{i}$ - referred to as twists - have been limited to $b_{i}=0$. Correspondingly, the elliptic curve in question has a single puncture only: $E_{\tau}^{\times}=\mathbb{C} /(\mathbb{Z}+\mathbb{Z} \tau) \backslash\{0\}$. Evaluating this subclass of elliptic iterated integrals at $z=1$ leads to the definition of Enriquez' $A$-cycle elliptic multiple zeta values or eMZVs for short:

$$
\begin{aligned}
\omega\left(n_{1}, n_{2}, \ldots, n_{\ell}\right) & =\int_{0 \leq z_{i} \leq z_{i+1} \leq 1} f^{\left(n_{1}\right)}\left(z_{1}\right) \mathrm{d} z_{1} f^{\left(n_{2}\right)}\left(z_{2}\right) \mathrm{d} z_{2} \ldots f^{\left(n_{\ell}\right)}\left(z_{\ell}\right) \mathrm{d} z_{\ell} \\
& =\Gamma\left(\begin{array}{cccc}
n_{\ell} & \ldots & n_{2} \\
0 & \ldots & n_{1} & 0
\end{array} ; 1\right)=\Gamma\left(n_{\ell}, \ldots, n_{2}, n_{1} ; 1\right) .
\end{aligned}
$$

The quantities $w=\sum_{i=1}^{\ell} n_{i}$, and the number $\ell$ of integrations in eqs. (2.1) and (2.4) are referred to as weight and length of the elliptic iterated integral and of the corresponding eMZV, respectively.

Allowing for rational values $s_{i}$ and $r_{i}$ in $b_{i}=s_{i}+r_{i} \tau$, leads to twisted elliptic multiple zeta values or teMZVs:

$$
\begin{aligned}
\omega\left(\begin{array}{c}
n_{1}, n_{2}, \ldots, n_{\ell} \\
b_{1}, b_{2}, \ldots, b_{\ell}
\end{array}\right) & =\int_{0 \leq z_{i} \leq z_{i+1} \leq 1} f^{\left(n_{1}\right)}\left(z_{1}-b_{1}\right) \mathrm{d} z_{1} f^{\left(n_{2}\right)}\left(z_{2}-b_{2}\right) \mathrm{d} z_{2} \ldots f^{\left(n_{\ell}\right)}\left(z_{\ell}-b_{\ell}\right) \mathrm{d} z_{\ell} \\
& =\Gamma\left(\begin{array}{llll}
n_{\ell} & n_{\ell-1} & \ldots & n_{1} \\
b_{\ell} & b_{\ell-1} & \ldots & b_{1}
\end{array} ;\right),
\end{aligned}
$$

where the notions of weight and length carry over from eq. (2.4) directly. Taking the doubleperiodicity $(2.3)$ of the weighting functions $f^{(n)}$ into account, one can limit the attention to the fundamental domain of the elliptic curve with $r_{i}, s_{i} \in[0,1)$, i.e. the shaded region in figure 2 .

In this article we are going to limit our attention to twists $\mathbb{Q}+\mathbb{Q} \tau$, that is $r_{i}, s_{i} \in \mathbb{Q}$. In 


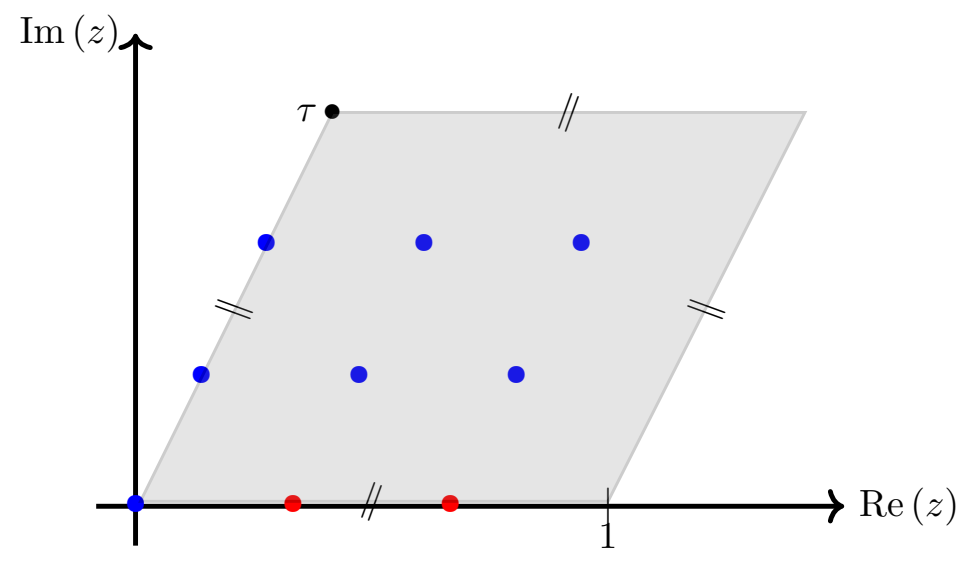

Figure 2: Example of the lattice $\Lambda_{N}+\Lambda_{N} \tau$ at $N=3$ : Proper rational twists and generic twists are marked in red and blue, respectively. Edges marked by $/ /$ and $\approx$ are identified in $\mathbb{C} /(\mathbb{Z}+\mathbb{Z} \tau)$, respectively.

order to classify those, let us introduce

$$
\Lambda_{N}=\left\{0, \frac{1}{N}, \frac{2}{N}, \ldots, \frac{N-1}{N}\right\}, \quad \Lambda_{N}^{\times}=\Lambda_{N} \backslash\{0\} .
$$

If $b_{i} \in \Lambda_{N}^{\times}$, the twist is referred to as proper rational. Correspondingly, all other twists - that is those with $b_{i} \in\left(\Lambda_{N}+\Lambda_{N} \tau\right) \backslash \Lambda_{N}^{\times}$as visualized in figure 2 - are called generic twists. While in the latter situation divergences occur at endpoints only and can be addressed using the methods in ref. [8], the presence of a proper rational twist requires more work as discussed in subsection 2.1.

Twisted eMZVs based on proper rational twists do not make an appearance in the openstring one-loop amplitude. However, they are interesting from a number-theoretic point of view because their constant terms give rise to cyclotomic generalizations of MZVs or "cyclotomic MZVs" for short $3,13-15$. The set of (generic) twists $b_{i} \in\{0, \tau / 2\}$ turns out to lead to teMZVs relevant for the non-planar open-string amplitude, which we are going to discuss in section 4.

\subsection{Regularization}

In order to regularize the divergences in eq. (2.5) caused by twists $b_{1}, \ldots, b_{\ell} \in \Lambda_{N}^{\times}$, we propose to replace the straight line $[0,1]$ by the domain of integration $[0,1]_{\varepsilon}$ in the right panel of figure 3 .
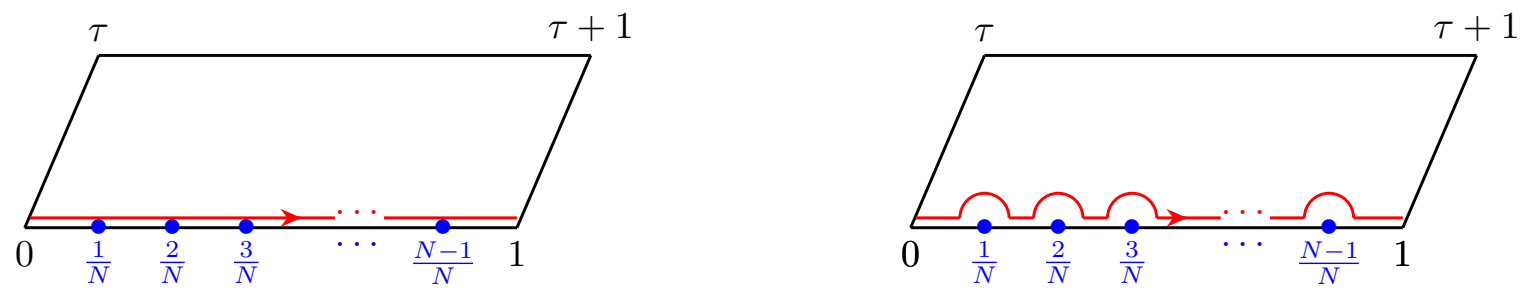

Figure 3: Deformation of the straight-line path $[0,1]$ to the path $[0,1]_{\varepsilon}$, avoiding the possible singularities of $f^{(1)}$.

Here, $\varepsilon>0$ is an additional real parameter, which determines the radii of the semicircles around 
the proper rational twists in figure 3 . One then defines regularized values of teMZVs

$$
\omega\left(\begin{array}{l}
n_{1}, n_{2}, \ldots, n_{\ell} \\
b_{1}, b_{2}, \ldots, b_{\ell}
\end{array}\right)=\lim _{\varepsilon \rightarrow 0} \int_{[0,1]_{\varepsilon}} f^{\left(n_{1}\right)}\left(z_{1}-b_{1}\right) \mathrm{d} z_{1} f^{\left(n_{2}\right)}\left(z_{2}-b_{2}\right) \mathrm{d} z_{2} \ldots f^{\left(n_{\ell}\right)}\left(z_{\ell}-b_{\ell}\right) \mathrm{d} z_{\ell},
$$

which agree with eq. (2.5) if all twists are generic. The existence of the limit in eq. (2.7) requires some explanation because $f^{(1)}\left(z-b_{i}\right)$ has a pole at $z=b_{i}$. For a single proper rational twist $b \in \Lambda_{N}^{\times}$, the path $[0,1]_{\varepsilon}$ can be written as the composition of a straight line from 0 to $b-\varepsilon$, followed by a semicircle from $b-\varepsilon$ to $b+\varepsilon$ above $b$, and then followed by a straight line from $b+\varepsilon$ to 1 :

$$
\omega\left(\begin{array}{l}
1 \\
b
\end{array}\right)=\lim _{\varepsilon \rightarrow 0} \int_{[0, b-\varepsilon]} \mathrm{d} z f^{(1)}(z-b)+\int_{[b-\varepsilon, b+\varepsilon]} \mathrm{d} z f^{(1)}(z-b)+\int_{[b+\varepsilon, 1]} \mathrm{d} z f^{(1)}(z-b) .
$$

Clearly, the contribution to eq. (2.8) coming from the non-holomorphic part $2 \pi i \frac{\operatorname{Im}(z)}{\operatorname{Im}(\tau)}$ of $f^{(1)}$ in eq. (2.2) vanishes in the limit $\varepsilon \rightarrow 0$. The contribution coming from the closed one-form $\frac{\theta_{1}^{\prime}(z, \tau)}{\theta_{1}(z, \tau)} \mathrm{d} z$ in turn is independent of $\varepsilon$ by Stokes' theorem, since the paths $[0,1]_{\varepsilon}$ belong to the same homotopy class. Computing the right hand side of eq. (2.8), we find that

$$
\omega\left(\begin{array}{l}
1 \\
b
\end{array}\right)=\log \left(\frac{\theta_{1}(1-b, \tau)}{\theta_{1}(b, \tau)}\right)-i \pi=-i \pi,
$$

where the first term comes from the first and third integral in eq. $(2.8)$ and vanishes by reflection and periodicity of the $\theta_{1}$ function. The contribution of $-i \pi$ is due to the second integral in eq. $(\overline{2.8})$, by the residue theorem.

The higher-length case is handled similarly. First we note that on the semicircles we have additional contributions from the non-holomorphic parts of the weighting functions $f^{\left(n_{i}\right)}\left(z_{i}-b_{i}\right)$ (cf. eq. (2.17) below), given by powers of $2 \pi i \frac{\operatorname{Im}\left(z_{i}-b_{i}\right)}{\operatorname{Im}(\tau)}$. These additional contributions on the semicircle are bounded by $\operatorname{Im}\left(z_{i}\right) \leq \varepsilon$, and the accompanying meromorphic functions have at most a simple pole at $b_{i}$. Hence, the overall integrand on the semicircle is finite as $\varepsilon \rightarrow 0$. Subsequently, we may use the composition of paths formula for iterated integrals (cf. eq. (C.3)) to check that the contributions from the non-holomorphic parts on the semicircles are in fact of order $\mathcal{O}(\varepsilon)$ and therefore do not contribute in the limit $\varepsilon \rightarrow 0$. Thus we are left with integrals over meromorphic functions of the $z_{i}$, which do not depend on $\varepsilon$ by homotopy of all paths $[0,1]_{\varepsilon}$.

The upshot is then that, up to terms which vanish in the limit $\varepsilon \rightarrow 0$, the right hand side of eq. (2.7) is independent of $\varepsilon$, thus convergent. An example at length two can be found in appendix C.

\subsection{General properties of elliptic iterated integrals and (t)eMZVs}

In general, iterated integrals of the form (2.1) satisfy shuffle relations. In terms of combined letters $B_{i}={ }_{b_{i}}^{n_{i}}$, the shuffle relation for elliptic iterated integrals reads

$$
\Gamma\left(B_{1}, B_{2}, \ldots, B_{\ell_{B}} ; z\right) \Gamma\left(C_{1}, \ldots, C_{\ell_{C}} ; z\right)=\Gamma\left(\left(B_{1}, B_{2}, \ldots, B_{\ell_{B}}\right) ш\left(C_{1}, \ldots, C_{\ell_{C}}\right) ; z\right),
$$

where $\amalg$ denotes the shuffle product [37]. Naturally, the shuffle relation eq. (2.10) straightforwardly carries over to eMZVs,

$$
\omega\left(n_{1}, n_{2}, \ldots, n_{\ell_{n}}\right) \omega\left(m_{1}, m_{2}, \ldots, m_{\ell_{m}}\right)=\omega\left(\left(n_{1}, n_{2}, \ldots, n_{\ell_{n}}\right) ш\left(m_{1}, m_{2}, \ldots, m_{\ell_{m}}\right)\right),
$$


and teMZVs

$$
\omega\left(B_{1}, B_{2}, \ldots, B_{\ell_{B}}\right) \omega\left(C_{1}, \ldots, C_{\ell_{C}}\right)=\omega\left(\left(B_{1}, B_{2}, \ldots, B_{\ell_{B}}\right) ш\left(C_{1}, \ldots, C_{\ell_{C}}\right)\right)
$$

where the $B_{i}, C_{i}$ are combined letters as defined above.

Taking into account the parity property $(2.3)$ of the weighting functions $f^{\left(n_{i}\right)}$ and the definition of elliptic iterated integrals, one finds the reflection identity

$$
\Gamma\left(\begin{array}{cccc}
n_{1} & n_{2} & \ldots & n_{\ell} \\
b_{1} & b_{2} & \ldots & b_{\ell}
\end{array} ; z\right)=(-1)^{n_{1}+n_{2}+\ldots+n_{\ell}} \Gamma\left(\begin{array}{ccccc}
n_{\ell} & \ldots & n_{2} & n_{1} \\
z-b_{\ell} & \ldots & z-b_{2} & z-b_{1}
\end{array} ; z\right)
$$

which is, however, valid only if the combined letter $B_{i}={ }_{b_{i}}^{1}$ with $b_{i}$ a proper rational twist does not occur. The need to exclude such instances of $B_{i}$ stems from the regularization of section 2.1 which does not preserve the reflection property.

Again, as in the case of the shuffle relation, there is an echo of the reflection identity for eMZVs and teMZVs:

$$
\begin{aligned}
\omega\left(n_{1}, n_{2}, \ldots, n_{\ell-1}, n_{\ell}\right) & =(-1)^{n_{1}+n_{2}+\ldots+n_{\ell}} \omega\left(n_{\ell}, n_{\ell-1}, \ldots, n_{2}, n_{1}\right) \\
\omega\left(\begin{array}{l}
n_{1}, n_{2}, \ldots, n_{\ell} \\
b_{1}, b_{2}, \ldots, b_{\ell}
\end{array}\right) & =(-1)^{n_{1}+n_{2}+\ldots+n_{\ell}} \omega\left(\begin{array}{c}
n_{\ell}, n_{\ell-1}, \ldots, n_{1} \\
\tilde{b}_{\ell}, \tilde{b}_{\ell-1}, \ldots, \tilde{b}_{1}
\end{array}\right),
\end{aligned}
$$

where $\tilde{b}_{i}$ is the representative of $-b_{i}$ in the fundamental domain of the elliptic curve and letters $B_{i}={ }_{b_{i}}^{1}$ with $b_{i}$ a proper rational twist are again excluded.

\section{$2.3 \quad q$-expansion of teMZVs}

In contrast to usual MZVs, which are just numbers, eMZVs and teMZVs are functions of the modular parameter $\tau$ and can be expanded in its exponentiated cousin $q=e^{2 \pi i \tau}$. The $q$ expansions of eMZVs and teMZVs rely on the available $q$-expansions of the weighting functions $f^{(n)}$. The discussion below will simplify considerably, if we consider in addition a class of meromorphic weighting functions $g^{(n)}$.

While the weighting functions $f^{(n)}$ appear as expansion coefficients of the doubly-periodic completion $\Omega(z, \alpha, \tau)$ of the Eisenstein-Kronecker series $F(z, \alpha, \tau)$ (cf. eq. (B.1)) $[6]$

$$
\Omega(z, \alpha, \tau)=\exp \left(2 \pi i \alpha \frac{\operatorname{Im}(z)}{\operatorname{Im}(\tau)}\right) F(z, \alpha, \tau)=\sum_{n=0}^{\infty} f^{(n)}(z, \tau) \alpha^{n-1},
$$

the functions $g^{(n)}$ are the expansion coefficients of the Eisenstein-Kronecker series [38, 39]

$$
F(z, \alpha, \tau)=\sum_{n=0}^{\infty} g^{(n)}(z, \tau) \alpha^{n-1}
$$

The set of meromorphic functions $g^{(n)}(z, \tau)$ starts with $g^{(0)}=1$ and $g^{(1)}(z, \tau)=\frac{\theta_{1}^{\prime}(z, \tau)}{\theta_{1}(z, \tau)}$ and can be related to their doubly-periodic but non-holomorphic ${ }^{4}$ completions via eq. $(2.15)$ :

$$
f^{(n)}(z, \tau)=\sum_{j=0}^{n} \frac{1}{(n-j) !}\left(2 \pi i \frac{\operatorname{Im}(z)}{\operatorname{Im}(\tau)}\right)^{n-j} g^{(j)}(z, \tau) .
$$

\footnotetext{
${ }^{4}$ Note that by Liouville's theorem, every meromorphic, doubly-periodic function, which has at most a simple pole at zero must be constant. Therefore, one either has to include non-holomorphic factors (as we do here, following [6]) or allow poles of order $\geq 2$ (as in 4], §5.1.2).
} 
Quasi-periodicity and the reflection property of $F(z, \alpha, \tau)$ (see eqs. (B.5) and (B.6)) imply the following properties of the $g^{(n)}(z, \tau)$,

$$
g^{(n)}(z)=g^{(n)}(z+1), \quad g^{(n)}(z+\tau)=\sum_{j=0}^{n} \frac{(-2 \pi i)^{j}}{j !} g^{(n-j)}(z), \quad g^{(n)}(-z)=(-1)^{n} g^{(n)}(z)
$$

and their Fourier expansions are given by $39,6,30$

$$
\begin{aligned}
g^{(1)}(z, \tau) & =\pi \cot (\pi z)-2 i(2 \pi i) \sum_{n, m=1}^{\infty} \sin (2 \pi m z) q^{m n} & & \\
g^{(2 k)}(z, \tau) & =-2 \zeta_{2 k}-2 \frac{(2 \pi i)^{2 k}}{(2 k-1) !} \sum_{n, m=1}^{\infty} \cos (2 \pi m z) n^{2 k-1} q^{m n}, & & k>0 \\
g^{(2 k+1)}(z, \tau) & =-2 i \frac{(2 \pi i)^{2 k+1}}{(2 k) !} \sum_{n, m=1}^{\infty} \sin (2 \pi m z) n^{2 k} q^{m n}, & & k>0
\end{aligned}
$$

For real values of $z$ one finds from eqs. (2.15) and $(2.17)$ that $f^{(n)}(z)=g^{(n)}(z)$ and their $q$-expansions agree. In particular, they can be employed to find $q$-expansions for eMZVs

$$
\omega\left(n_{1}, n_{2}, \ldots, n_{\ell}\right)=\omega_{0}\left(n_{1}, n_{2}, \ldots, n_{\ell}\right)+\sum_{k=1}^{\infty} c_{k}\left(n_{1}, n_{2}, \ldots, n_{\ell}\right) q^{k}
$$

The $q$-independent quantity $\omega_{0}$ in eq. $(\overline{2.20})$ is called the constant term of the eMZV $\omega$ and is known to be a $\mathbb{Q}\left[(2 \pi i)^{-1}\right]$-linear combination of MZVs (see refs. $\left.30,10,11\right)$.

In order to describe the $q$-dependence of teMZVs in a similar manner, we consider the twist $b=s+r \tau \in \Lambda_{N}+\Lambda_{N} \tau$ in the weighting function $f^{(n)}(z-b)$ eq. (2.17) for real values of $z$ :

$$
f^{(n)}(z-s-r \tau, \tau)=\sum_{j=0}^{n} \frac{(-2 \pi i r)^{n-j}}{(n-j) !} g^{(j)}(z-s-r \tau, \tau), \quad z \in \mathbb{R} .
$$

Employing eqns. (2.19), the functions $g^{(j)}(z-b, \tau)$, can be expanded in non-negative rational powers of $q$,

$$
\begin{aligned}
& g^{(2 k+1)}(z-s-r \tau, \tau)=\delta_{k, 0} \pi \cot (\pi(z-s-r \tau))+\frac{(2 \pi i)^{2 k}}{(2 k) !} \sum_{m, n=1}^{\infty} n^{2 k} q^{m n} \\
& \quad \times\left\{\cos (2 \pi m(z-s))\left(q^{m r}-q^{-m r}\right)-i \sin (2 \pi m(z-s))\left(q^{m r}+q^{-m r}\right)\right\}, \quad k \geq 0 \\
& g^{(2 k)}(z-s-r \tau, \tau)=-2 \zeta_{2 k}-\frac{(2 \pi i)^{2 k-1}}{(2 k-1) !} \sum_{m, n=1}^{\infty} n^{2 k-1} q^{m n} \\
& \quad \times\left\{\cos (2 \pi m(z-s))\left(q^{m r}+q^{-m r}\right)-i \sin (2 \pi m(z-s))\left(q^{m r}-q^{-m r}\right)\right\}, \quad k>0 .
\end{aligned}
$$

If $0<r<1$, i.e. if $b$ is a generic twist, then the cotangent term in $g^{(1)}$ may be rewritten as

$$
\pi \cot (\pi(z-s-r \tau))=i \pi\left(1+q^{r} e^{2 \pi i(s-z)}\right) \sum_{n=0}^{\infty}\left(q^{r} e^{2 \pi i(s-z)}\right)^{n} .
$$

On these grounds, $f^{(n)}(z-s-r \tau)$ can be expanded in powers of $q^{r}$ and $q^{1-r}$ such that every 
teMZV admits an expansion in $q^{p}$,

$$
\omega\left(\begin{array}{l}
n_{1}, n_{2}, \ldots, n_{\ell} \\
b_{1}, b_{2}, \ldots, b_{\ell}
\end{array}\right)=\omega_{0}\left(\begin{array}{c}
n_{1}, n_{2}, \ldots, n_{\ell} \\
b_{1}, b_{2}, \ldots, b_{\ell}
\end{array}\right)+\sum_{k=1}^{\infty} c_{k}\left(\begin{array}{c}
n_{1}, n_{2}, \ldots, n_{\ell} \\
b_{1}, b_{2}, \ldots, b_{\ell}
\end{array}\right)\left(q^{p}\right)^{k}
$$

where $1 / p \in \mathbb{Q}$ is the least common denominator of all occurring $r_{i}$. The $q$-independent quantity $\omega_{0}$ in eq. (2.24) is called the constant term of the teMZV, which we are going to study in section 3. Depending on the set of twists $b_{i}$, different classes of objects appear as constant terms: while MZVs cover constant terms for generic twists, proper rational twists lead to cyclotomic MZVs $\mid 3,13$. We will refer to teMZVs for which $c_{k}\left(\begin{array}{c}n_{1}, n_{2}, \ldots, n_{\ell} \\ b_{1}, b_{2}, \ldots, b_{\ell}\end{array}\right)=0$ for all $k \in \mathbb{N}^{+}$as constant.

\section{$3 \quad q$-expansion for twisted elliptic multiple zeta values}

The goal of this section is to set up an initial value problem for teMZVs eq. (2.5) and to obtain their $q$-expansion without performing any integral over their trigonometric constituents in eq. (2.22). The differential equation to be derived in this section will prove to be the main tool to allow the efficient computation and comparison of teMZVs in a convenient representation as iterated integrals. In particular, this representation will prove useful in the context of calculating non-planar contributions to the one-loop amplitude in section 4 .

Following the strategy for computing the usual eMZV's $q$-expansion in [8, 10], in a first step we derive a first-order differential equation in $\tau$ for teMZVs. In the second step, a boundary value at the cusp $\tau \rightarrow i \infty$ will be determined to identify a unique solution to the differential equation. Since the action of $\partial_{\tau}$ reduces the length of teMZVs, one can derive the $q$-expansion for teMZVs recursively.

For eMZVs, classical Eisenstein series and MZVs are the building blocks for the $\tau$-derivative and constant term respectively [8,10,11]. Similarly, we will show that the weighting functions $f^{(n)}(b, \tau)$ evaluated at lattice points $b \in \Lambda_{N}+\Lambda_{N} \tau$ and cyclotomic MZVs are suitable generalizations thereof for teMZVs.

After deriving the differential equation in subsection 3.1, the constant term will be elaborated on in subsection 3.2 for generic twists and modifications when including proper rational twists are discussed in subsection 3.3.

\subsection{Differential equation}

We begin by defining a generating series for teMZVs of length $\ell$,

$$
\begin{aligned}
\mathrm{T}\left[\begin{array}{c}
\alpha_{1}, \alpha_{2}, \ldots, \alpha_{\ell} \\
b_{1}, b_{2}, \ldots, b_{\ell}
\end{array}\right] & =\int_{0 \leq z_{i} \leq z_{i+1} \leq 1} \Omega\left(z_{1}-b_{1}, \alpha_{1}, \tau\right) \mathrm{d} z_{1} \Omega\left(z_{2}-b_{2}, \alpha_{2}, \tau\right) \mathrm{d} z_{2} \ldots \Omega\left(z_{\ell}-b_{\ell}, \alpha_{\ell}, \tau\right) \mathrm{d} z_{\ell} \\
& =\sum_{n_{1}, n_{2}, \ldots, n_{\ell}=0}^{\infty} \alpha_{1}^{n_{1}-1} \alpha_{2}^{n_{2}-1} \ldots \alpha_{\ell}^{n_{\ell}-1} \omega\left(\begin{array}{l}
n_{1}, n_{2}, \ldots, n_{\ell} \\
b_{1}, b_{2}, \ldots, b_{\ell}
\end{array}\right),
\end{aligned}
$$

generalizing a construction of ref. [8]. For simplicity, we will assume in this subsection that the twists $b_{i}=s_{i}+r_{i} \tau$ are generic, i.e $r_{i} \in(0,1)$. The case of proper rational twists is discussed in appendix $\mathrm{F}$.

First, since the domain of integration in eq. $(3.1)$ is the interval $[0,1] \subset \mathbb{R}$, it is natural to restrict the function $z \mapsto \Omega(z-b, \alpha, \tau)$ to real arguments of $z$. With this restriction, the 
following differential equation is then a consequence of the mixed heat equation (B.4)

$$
\begin{aligned}
\partial_{\tau} \Omega(z-s-r \tau, \alpha, \tau) & =\exp (-2 \pi i r \alpha) \partial_{\tau} F(z-s-r \tau, \alpha, \tau) \\
& =\exp (-2 \pi i r \alpha)\left(-r \partial_{z}+\frac{1}{2 \pi i} \partial_{z} \partial_{\alpha}\right) F(z-s-r \tau, \alpha, \tau) \\
& =\frac{1}{2 \pi i} \partial_{z} \partial_{\alpha} \Omega(z-s-r \tau, \alpha, \tau) .
\end{aligned}
$$

Here the partial derivative $\partial_{\tau}$ is understood to act on all occurrences of the variable $\tau$. Furthermore, we have used that $\partial_{z} r=\partial_{\tau} r=0$, since the twist $b=s+r \tau$ is fixed, and therefore neither depends on $z$ nor $\tau$. Note that in going from the first to the second line in eq. $(3.2)$, the term $r \partial_{z}$ appears by taking the occurrence of $\tau$ in the first argument of the Kronecker series into account. However, this additional term gets neatly absorbed when returning to the doubly-periodic completion $\Omega(z-s-r \tau, \alpha, \tau)$ in the last line.

The $\tau$-derivative of the generating function in eq. (3.1) reads

$$
\begin{aligned}
& 2 \pi i \frac{\partial}{\partial \tau} \mathrm{T}\left[\begin{array}{l}
\alpha_{1}, \alpha_{2}, \ldots, \alpha_{\ell} \\
b_{1}, b_{2}, \ldots, b_{\ell}
\end{array}\right]=\int_{0 \leq z_{i} \leq z_{i+1} \leq 1} \mathrm{~d} z_{1} \mathrm{~d} z_{2} \ldots \mathrm{d} z_{\ell} \sum_{i=1}^{\ell} \partial_{z_{i}} \partial_{\alpha_{i}} \Omega\left(z_{i}-b_{i}, \alpha_{i}\right) \prod_{j \neq i}^{\ell} \Omega\left(z_{j}-b_{j}, \alpha_{j}\right)
\end{aligned}
$$

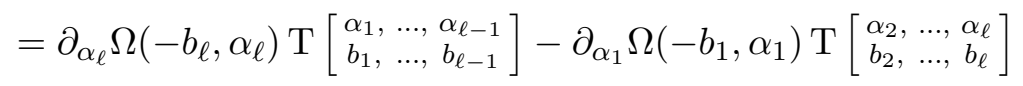

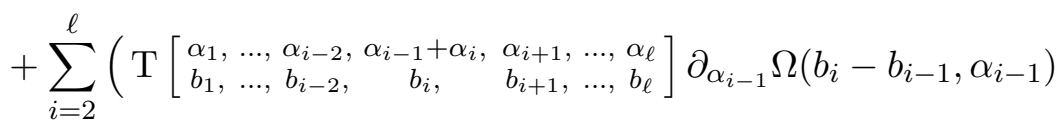

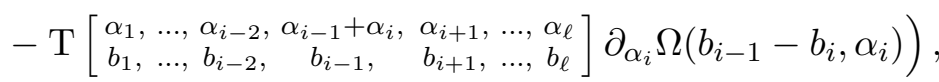

where we used eq. (3.2) in the first line and suppressed the dependence of $\mathrm{T}$ and $\Omega$ on the modular parameter $\tau$. In the second equality, the number of integrations is reduced by evaluating $\int \mathrm{d} z_{i} \partial_{z_{i}} \partial_{\alpha_{i}} \Omega\left(z_{i}-b_{i}, \alpha_{i}\right)$ via boundary terms $\left.\partial_{\alpha_{i}} \Omega\left(z_{i}-b_{i}, \alpha_{i}\right)\right|_{z_{i-1}} ^{z_{i+1}}$ with $z_{0}=0$ and $z_{\ell+1}=1$. The resulting products of the form $\Omega\left(z_{i}-b_{i-1}, \alpha_{i-1}\right) \Omega\left(z_{i}-b_{i}, \alpha_{i}\right)$ are rewritten using the Fay identity eq. (B.3) such that each integration variable $z_{i}$ appears in at most one factor of $\Omega$. The details of the computation can be found in appendix $\mathrm{E}$.

Upon expanding $\Omega$ and T in eq. (3.3) in $\alpha_{i}$, one can compare the coefficients of the monomials $\alpha_{1}^{m_{1}} \ldots \alpha_{\ell}^{m_{\ell}}$. The coefficient of each monomial is a linear combination of some $f^{(n)}$ multiplied by teMZVs of length $\ell-1$. Working out the details yields the following differential equation for teMZVs $(\ell \geq 2)$, and using the shorthand

$$
h^{(n)}(u, \tau)=(n-1) f^{(n)}(u, \tau) .
$$

for $u \in \mathbb{C} /(\mathbb{Z}+\mathbb{Z} \tau)$, we find

$$
\begin{aligned}
& 2 \pi i \partial_{\tau} \omega\left(\begin{array}{c}
n_{1}, \ldots, n_{\ell} \\
b_{1}, \ldots, b_{\ell}
\end{array}\right)=h^{\left(n_{\ell}+1\right)}\left(-b_{\ell}\right) \omega\left(\begin{array}{c}
n_{1}, \ldots, n_{\ell-1} \\
b_{1}, \ldots, b_{\ell-1}
\end{array}\right)-h^{\left(n_{1}+1\right)}\left(-b_{1}\right) \omega\left(\begin{array}{c}
n_{2}, \ldots, n_{\ell} \\
b_{2}, \ldots, b_{\ell}
\end{array}\right)
\end{aligned}
$$

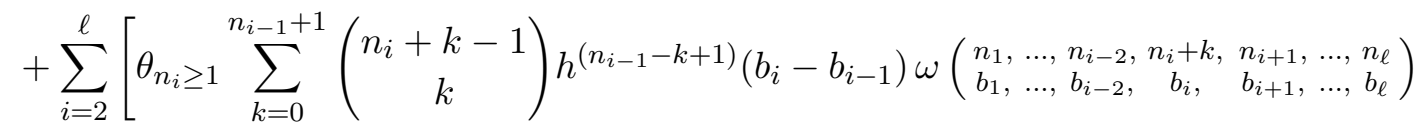

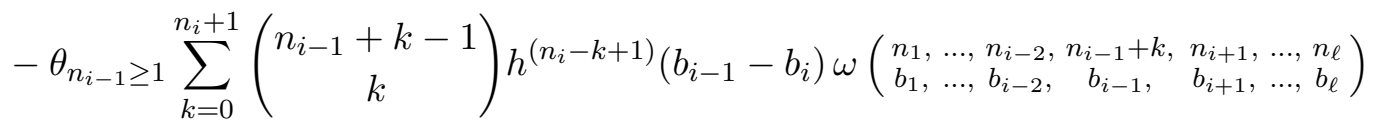




$$
\left.+(-1)^{n_{i}+1} \theta_{n_{i-1} \geq 1} \theta_{n_{i} \geq 1} h^{\left(n_{i-1}+n_{i}+1\right)}\left(b_{i}-b_{i-1}\right) \omega\left(\begin{array}{c}
n_{1}, \ldots, n_{i-2}, 0, n_{i+1}, \ldots, n_{\ell} \\
b_{1}, \ldots, b_{i-2}, 0, b_{i+1}, \ldots, b_{\ell}
\end{array}\right)\right],
$$

where we have introduced $\theta_{n \geq 1}=1-\delta_{n, 0}$ for non-negative $n$ to indicate that some of the contributions in the last three lines vanish for $n_{i}=0$. For vanishing twists $b_{i}=0$, eq. (3.5) reduces to the differential equation for eMZVs stated in eq. (2.47) of ref. 10 since the weighting functions $f^{(n)}$ are related to holomorphic Eisenstein series (with $\mathrm{G}_{0}(\tau)=-1$ ) via

$$
-\lim _{z \rightarrow 0} f^{(k)}(z, \tau)=\mathrm{G}_{k}(\tau)=\left\{\begin{aligned}
2 \zeta_{k}+\frac{2(2 \pi i)^{k}}{(k-1) !} \sum_{m, n=1}^{\infty} m^{k-1} q^{m n} & : k \geq 2 \text { even } \\
-1 & : k=0 \\
0 & : k \neq 1 \text { odd }
\end{aligned}\right.
$$

where the limit is understood to be taken along the real axis. In other words, the functions $h^{(n)}(b, \tau)$ occurring in the differential equation (3.5) (which are modular forms for congruence subgroups of $\mathrm{SL}_{2}(\mathbb{Z})$ ) take the rôle of Eisenstein series in the differential equation for eMZVs. Also, note that the exceptional case $f^{(1)}(z, \tau)$ (which has a pole at $z=0$ ) does not appear in eq. (3.3) since it is accompanied by $\alpha^{0}=1$ in the generating series $\Omega(z, \alpha, \tau)$ and is therefore annihilated upon application of the $\alpha$-derivative.

\subsection{Constant terms for generic twists}

In this subsection we are going to extend the constant-term procedure for eMZVs studied in 10,11 to a procedure delivering the constant terms for teMZVs. Calculating the constant term for teMZVs amounts to the computation of the limit $\tau \rightarrow i \infty$ of eq. (2.5). This limit will figure as the initial value for the differential equation (3.5) discussed in the previous subsection.

In order to make the bookkeeping more efficient, it is convenient to consider a suitable generating series of teMZVs, which is a generalization of the $A$-part of Enriquez' elliptic KZB associator 7 to the realm of teMZVs: More precisely, for every $N \geq 1$ we will consider a formal power series in nested commutators

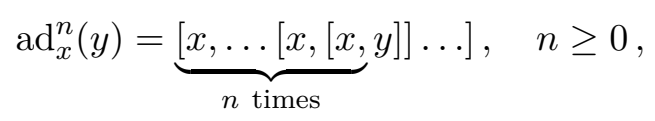

of the non-commutative variables $y,\left\{x_{b_{i}}\right\}_{b_{i} \in\left(\Lambda_{N}+\Lambda_{N} \tau\right) \backslash \Lambda_{N}^{\times}}$as follows:

$$
\begin{aligned}
A_{\left(\Lambda_{N}+\Lambda_{N} \tau\right) \backslash \Lambda_{N}^{\times}}^{\text {twist }}(\tau) & =\sum_{\ell \geq 0}(-1)^{\ell} \sum_{\begin{array}{c}
n_{1}, n_{2}, \ldots, n_{\ell} \geq 0 \\
b_{1}, b_{2}, \ldots, b_{\ell} \in\left(\Lambda_{N}+\Lambda_{N} \tau\right) \backslash \Lambda_{N}^{\times}
\end{array}} \omega\left(\begin{array}{c}
n_{1}, n_{2}, \ldots, n_{\ell} \\
b_{1}, b_{2}, \ldots, b_{\ell}
\end{array}\right) \operatorname{ad}_{x_{b_{\ell}}}^{n_{\ell}}(y) \ldots \operatorname{ad}_{x_{b_{2}}}^{n_{2}}(y) \operatorname{ad}_{x_{b_{1}}}^{n_{1}}(y) \\
& =\tilde{\mathcal{P}} \exp \left(-\int_{0}^{1} \mathrm{~d} z \sum_{k=0}^{\infty} \sum_{b \in\left(\Lambda_{N}+\Lambda_{N} \tau\right) \backslash \Lambda_{N}^{\times}} f^{(k)}(z-b, \tau) \operatorname{ad}_{x_{b}}^{k}(y)\right),
\end{aligned}
$$

where $\Lambda_{N}$ and $\Lambda_{N}^{\times}$were defined in eq. $(2.6)$, and $\tilde{\mathcal{P}} \exp (\ldots)$ denotes the path-ordered exponential with reverted order of multiplication for the non-commutative variables in comparison to the order of the integration variables ${ }^{5} z$. We note that there is no loss of generality in studying the lattice $\Lambda_{N}+\Lambda_{N} \tau$ rather than $\Lambda_{N}+\Lambda_{M} \tau$ with $M \neq N$ : the latter can be embedded into the

\footnotetext{
${ }^{5}$ Although composed of several non-commutative variables $x_{b_{i}}$ and $y$, each nested commutator $\operatorname{ad}_{x_{b_{i}}}^{n_{i}}(y)$ is treated as a single letter when reversing the order of multiplication.
} 
lattice $\Lambda_{N^{\prime}}+\Lambda_{N^{\prime}} \tau$ with $N^{\prime}$ the least common multiple of $M$ and $N$. Also, proper rational twists $b \in \Lambda_{N}^{\times}$have been excluded from the summation range for $b$ in eq. (3.8) in order to relegate a discussion of the additional ingredients required in these cases to section 3.3 .

The series (3.8) combines different instances of the generating series $\mathrm{T}\left[\begin{array}{cccc}\alpha_{1} & \alpha_{2} & \ldots & \alpha_{\ell} \\ b_{1} & b_{2} & \ldots & b_{\ell}\end{array}\right]$ in eq. (3.1),

$$
A_{\left(\Lambda_{N}+\Lambda_{N} \tau\right) \backslash \Lambda_{N}^{\times}}^{\mathrm{twist}}(\tau) \longleftrightarrow \sum_{\ell \geq 0}(-1)^{\ell} \sum_{b_{1}, \ldots, b_{\ell} \in\left(\Lambda_{N}+\Lambda_{N} \tau\right) \backslash \Lambda_{N}^{\times}} \mathrm{T}\left[\begin{array}{lllll}
\alpha_{\ell} & \alpha_{\ell-1} & \ldots & \alpha_{1} \\
b_{\ell} & b_{\ell-1} & \ldots & b_{1}
\end{array}\right],
$$

summing over all values of the length $\ell \geq 0$ and the generic twists $b_{i} \in\left(\Lambda_{N}+\Lambda_{N} \tau\right) \backslash \Lambda_{N}^{\times}$. The noncommutative product of $\operatorname{ad}_{x_{b_{i}}}^{k_{i}}(y)$ corresponds to commutative variables $\alpha_{i}^{k_{i}-1}$ in eq. (3.1), which accompany individual teMZVs in the respective generating series. While the organization via $\alpha_{i}^{k_{i}-1}$ is better suited for the study of the differential equation of teMZVs, the non-commutative variables $\operatorname{ad}_{x_{b_{i}}}^{k_{i}}(y)$ in eq. (3.7) are well adapted to the subsequent analysis of their constant terms 6 .

\subsubsection{Degeneration of weighting functions}

In order to compute $\lim _{\tau \rightarrow i \infty} A_{\left(\Lambda_{N}+\Lambda_{N} \tau\right) \backslash \Lambda_{N}^{\times}}^{\text {twist }}(\tau)$, we need to study the degeneration of the weighting functions $f^{(k)}(z-b, \tau)$ as $\tau \rightarrow i \infty$ (or equivalently $q \rightarrow 0$ ). Conveniently, the limit is expressed in the variables

$$
w=e^{2 \pi i z}, \quad \mathrm{~d} z=\frac{1}{2 \pi i} \frac{\mathrm{d} w}{w} .
$$

Using the $q$-expansions eqs. $(2.22)$ and $(\overline{2.23})$ together with eq. $(2.21)$ we obtain, for generic twists and $k>1$,

$$
\begin{aligned}
\lim _{\tau \rightarrow i \infty} f^{(k)}(z-s-r \tau, \tau) \mathrm{d} z & =\left(\frac{\pi i(-2 \pi i r)^{k-1}}{(k-1) !}-2 \sum_{m=0}^{\left\lfloor\frac{k}{2}\right\rfloor} \frac{(-2 \pi i r)^{k-2 m}}{(k-2 m) !} \zeta_{2 m}\right) \frac{1}{2 \pi i} \frac{\mathrm{d} w}{w} \\
& =-\frac{\mathrm{d} w}{w} \sum_{m=0}^{k} \frac{B_{m}(-2 \pi i)^{m-1}}{m !} \frac{(-2 \pi i r)^{k-m}}{(k-m) !}, \quad k>1 .
\end{aligned}
$$

Here, we have used $\zeta_{2 m}=-\frac{B_{2 m}(2 \pi i)^{2 m}}{2(2 m) !}$, where $B_{k}$ denotes the $k^{\text {th }}$ Bernoulli number (such that $\left.B_{1}=-\frac{1}{2}\right)$. While $f^{(0)}=1$, the case of $f^{(1)}(z-b)$ is special and we find

$$
\lim _{\tau \rightarrow i \infty} f^{(1)}(z-s-r \tau, \tau) \mathrm{d} z=\left\{\begin{array}{cl}
\left(\frac{1}{2}-r\right) \frac{\mathrm{d} w}{w} & : r \neq 0 \\
-\frac{1}{2} \frac{\mathrm{d} w}{w}+\frac{\mathrm{d} w}{w-1} & : r=s=0
\end{array} .\right.
$$

Combining eqs. (3.11) and (3.12) allows to rewrite the exponent of eq. (3.8) as follows:

$$
\lim _{\tau \rightarrow i \infty}-\mathrm{d} z \sum_{k=0}^{\infty}\left(\sum_{b \in\left(\Lambda_{N}+\Lambda_{N} \tau\right) \backslash \Lambda_{N}^{\times}} f^{(k)}(z-b, \tau) \operatorname{ad}_{x_{b}}^{k}(y)\right)=\tilde{y}_{N} \frac{\mathrm{d} w}{w}+t \frac{\mathrm{d} w}{w-1},
$$

\footnotetext{
${ }^{6}$ The use of two, essentially equivalent, generating series of teMZVs goes back to Enriquez' original work on eMZVs 8 .
} 
where

$$
\tilde{y}_{N}=-\frac{\operatorname{ad}_{x_{0}}}{e^{2 \pi i \operatorname{ad}_{x_{0}}-1}}(y)+\sum_{b \in\left(\Lambda_{N}+\Lambda_{N} \tau\right) \backslash \Lambda_{N}} \frac{\operatorname{ad}_{x_{b}} e^{-2 \pi i r \operatorname{ad}_{x_{b}}}}{e^{-2 \pi i \operatorname{ad}_{x_{b}}-1}}(y), \quad t=\left[y, x_{0}\right] .
$$

These definitions of $\tilde{y}_{N}$ and $t$ are tailored to track the appearance of the forms $\frac{\mathrm{d} w}{w}$ and $\frac{\mathrm{d} w}{w-1}$ in the degeneration limits eqs. (3.11) and (3.12) of $f^{(k)}(z-b, \tau)$. In absence of twists, for instance, the first contribution to $\tilde{y}_{N}$ in eq. (3.14) stems from setting $r=0$ in eq. (3.11) and identifying the generating series of $\frac{B_{k}(-2 \pi i)^{k-1}}{k !} \operatorname{ad}_{x_{0}}^{k}(y)$ :

$$
\begin{aligned}
\lim _{\tau \rightarrow i \infty}-\mathrm{d} z \sum_{k=0}^{\infty}\left(f^{(k)}(z, \tau) \operatorname{ad}_{x_{0}}^{k}(y)\right) & =-\frac{\mathrm{d} w}{w} \sum_{k=0}^{\infty} \frac{B_{k}(2 \pi i)^{k-1}}{k !} \operatorname{ad}_{x_{0}}^{k}(y)-\frac{\mathrm{d} w}{w-1} \operatorname{ad}_{x_{0}}(y) \\
& =-\frac{\operatorname{ad}_{x_{0}}}{e^{2 \pi i \operatorname{ad}_{x_{0}}-1}}(y) \frac{\mathrm{d} w}{w}-\frac{\mathrm{d} w}{w-1} \operatorname{ad}_{x_{0}}(y) .
\end{aligned}
$$

In the generalization to non-zero twists $r \neq 0$, the second contribution to $\tilde{y}_{N}$ in eq. (3.14) arises as the generating series of $\sum_{m=0}^{k} \frac{B_{m}(-2 \pi i)^{m-1}}{m !} \frac{(-2 \pi i r)^{k-m}}{(k-m) !} \operatorname{ad}_{x_{b}}^{k}(y)$. Note in particular that the dependence on $r$ gives rise to the factor of $e^{-2 \pi i r a x_{x_{b}}}$ in the numerator.

By comparing the degeneration behaviour in eq. (3.13) with eq. (3.8), we deduce that

$$
\lim _{\tau \rightarrow i \infty} A_{\left(\Lambda_{N}+\Lambda_{N} \tau\right) \backslash \Lambda_{N}^{\times}}^{\mathrm{twist}}(\tau)=\tilde{\mathcal{P}} \exp \left(\int_{C_{0}^{2 \pi}(1)}\left[\frac{\tilde{y}_{N}}{w}+\frac{t}{w-1}\right] \mathrm{d} w\right),
$$

where the unit circle $w \in C_{0}^{2 \pi}(1)$ arises from the path of integration $[0,1] \subset \mathbb{C}$ under the change of variables eq. (3.10). Strictly speaking, eq. (3.16) requires regularization, due to divergences at $w=1$. They are treated in analogy to eMZVs as described in refs. $[8,11,30$ and cause modifications to be pointed out in the subsequent discussion.

\subsubsection{Deforming the integration contour}

We have expressed $\lim _{\tau \rightarrow i \infty} A_{\left(\Lambda_{N}+\Lambda_{N} \tau\right) \backslash \Lambda_{N}^{\times}}^{\text {twist }}(\tau)$ as a generating series of iterated integrals of explicit differential forms along the unit circle $C_{0}^{2 \pi}(1)$, see the left panel of figure 4 below. Although the functions $\lim _{\tau \rightarrow i \infty} f^{(k)}(z-b, \tau)$ in eqs. (3.11) and (3.12) were restricted to real values of $z$, they can be extended to the doubly-punctured complex plane $z \in \mathbb{C} \backslash\{0,1\}$ (with $r$ kept constant). In this way, the integrand in eq. (3.13) is holomorphic on $\mathbb{C} \backslash\{0,1\}$, and the resulting path-ordered exponential eq. (3.16) is homotopy-invariant. Therefore one can replace the unit circle by a contour homotopic to it, visualized in the right panel of figure 4 .

This deformed contour can in turn be viewed as the composition of straight paths $P_{1}, P_{1}^{-1}$ connecting the points $w=0,1$ along with a circle $C_{0}^{2 \pi}(\varepsilon)$ of infinitesimal radius around the origin, as shown in figure 4. Moreover, the regularization alluded to above manifests itself in figure 4 both paths $C_{0}^{2 \pi}(1)$ as well as the composition $P_{1}^{-1} C_{0}^{2 \pi}(\varepsilon) P_{1} \hat{C}_{\pi}^{0}(\varepsilon)$ have to leave $w=1$ with velocity -1 and arrive back at $w=1$ with the same velocity. More precisely, both paths, which really are smooth functions $[0,1] \rightarrow \mathbb{C}^{\times}$must have a derivative equal to $-\frac{\partial}{\partial w} \in T_{1}\left(\mathbb{C}^{\times}\right)$, where $T_{1}$ denotes the tangent space at 1 . This is also the reason for the semicircle $\hat{C}_{\pi}^{0}(\varepsilon)$.

The virtue of deforming the path of integration is that eq. (3.16) can now be computed rather explicitly. First, in view of the reversal operation contained in the definition of $\tilde{\mathcal{P}}$, the composition of paths $\alpha$ and $\beta$ translates into a concatenation of the non-commutative series 

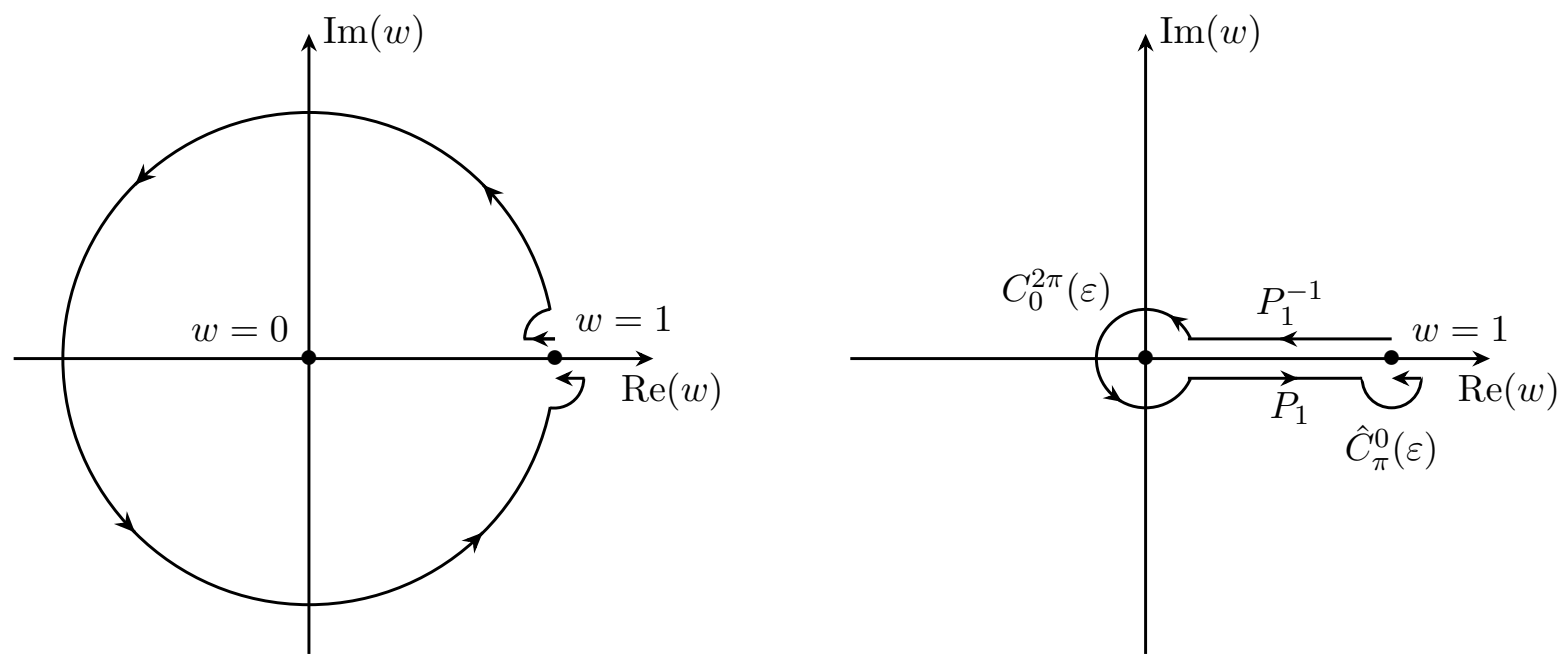

Figure 4: Deformation of the unit circle $C_{0}^{2 \pi}(1)$ to the path composition $P_{1}^{-1} C_{0}^{2 \pi}(\varepsilon) P_{1} \hat{C}_{\pi}^{0}(\varepsilon)$.

with reversed order,

$$
\tilde{\mathcal{P}} \exp \left(\int_{\alpha \beta} \omega\right)=\tilde{\mathcal{P}} \exp \left(\int_{\beta} \omega\right) \tilde{\mathcal{P}} \exp \left(\int_{\alpha} \omega\right)
$$

regardless of the differential form $\omega$. Hence, the equality of homotopy classes of paths (relative to the tangent vector $-\frac{\partial}{\partial w}$ at 1$)\left[C_{0}^{2 \pi}(1)\right]=\left[P_{1}^{-1} C_{0}^{2 \pi}(\varepsilon) P_{1} \hat{C}_{\pi}^{0}(\varepsilon)\right]$ allows to rewrite eq. (3.16) as

$$
\lim _{\tau \rightarrow i \infty} A_{\left(\Lambda_{N}+\Lambda_{N} \tau\right) \backslash \Lambda_{N}^{\times}}^{\mathrm{twist}}(\tau)=e^{i \pi t} \Phi\left(\tilde{y}_{N}, t\right) e^{2 \pi i \tilde{y}_{N}} \Phi\left(\tilde{y}_{N}, t\right)^{-1},
$$

where $\Phi\left(\tilde{y}_{N}, t\right)$ denotes the Drinfeld associator $[40$. In deducing eq. (3.18), we have used the identities

$$
\begin{aligned}
\Phi\left(\tilde{y}_{N}, t\right) & =\tilde{\mathcal{P}} \exp \left(\int_{P_{1}}\left[\frac{\tilde{y}_{N}}{w}+\frac{t}{w-1}\right] \mathrm{d} w\right), \\
e^{2 \pi i \tilde{y}_{N}} & =\tilde{\mathcal{P}} \exp \left(\int_{C_{0}^{2 \pi}(\varepsilon)}\left[\frac{\tilde{y}_{N}}{w}+\frac{t}{w-1}\right] \mathrm{d} w\right), \\
e^{i \pi t} & =\tilde{\mathcal{P}} \exp \left(\int_{\hat{C}_{\pi}^{0}(\varepsilon)}\left[\frac{\tilde{y}_{N}}{w}+\frac{t}{w-1}\right] \mathrm{d} w\right) .
\end{aligned}
$$

Since the coefficients of $\Phi$ are $\mathbb{Q}$-linear combinations of MZVs [41], an implementation of eq. (3.18) using a standard computer algebra system can be used to explicitly write the constant terms of teMZVs for generic twists as $\mathbb{Q}\left[(2 \pi i)^{-1}\right]$-linear combinations of MZVs. Equation (3.18) is a generalization of a similar formalism for eMZVs, which has been established in refs. [7. (see also $[10]$. Examples for constant terms of teMZVs computed via eq. (3.18) are gathered in appendix G.1.

\subsection{Constant terms for all twists}

So far, we have only considered the constant terms of teMZVs with generic twists. The presence of proper rational twists $b \in \Lambda_{N}^{\times}$requires a separate discussion due to the additional features of the corresponding weighting function $f^{(1)}(z-b, \tau)$ : 
- its simple pole in the interior of the domain of integration requires the regularization procedure of subsection 2.1

- its $\tau \rightarrow i \infty$ limit introduces singularities $\frac{\mathrm{d} w}{w-\zeta}$ with $\zeta$ denoting a root of unity

In order to facilitate the computation of the constant terms of teMZVs including proper rational twists, we again introduce a generating series for bookkeeping purposes

$$
\begin{aligned}
A_{\Lambda_{N}+\Lambda_{N} \tau}^{\mathrm{twist}}(\tau)= & \sum_{\ell \geq 0}(-1)^{\ell} \sum_{\substack{n_{1}, n_{2}, \ldots, n_{\ell} \geq 0 \\
b_{1}, b_{2}, \ldots, b_{\ell} \in \Lambda_{N}+\Lambda_{N} \tau}} \omega\left(\begin{array}{l}
n_{1}, n_{2}, \ldots, n_{\ell} \\
b_{1}, b_{2}, \ldots, b_{\ell}
\end{array}\right) \operatorname{ad}_{x_{b_{\ell}}}^{n_{\ell}}(y) \ldots \operatorname{ad}_{x_{b_{2}}}^{n_{2}}(y) \operatorname{ad}_{x_{b_{1}}}^{n_{1}}(y) \\
= & \lim _{\varepsilon \rightarrow 0} \tilde{\mathcal{P}} \exp \left(-\int_{[0,1]_{\varepsilon}} \mathrm{d} z \sum_{k=0}^{\infty} \sum_{b \in \Lambda_{N}+\Lambda_{N} \tau} f^{(k)}(z-b, \tau) \operatorname{ad}_{x_{b}}^{k}(y)\right)
\end{aligned}
$$

which generalizes eq. (3.8) to the case of arbitrary twists.

\subsubsection{Degeneration of weighting functions}

As in the above situation we need to determine the degeneration limit of the weighting functions $f^{(k)}(z-b, \tau)$ as $\tau \rightarrow i \infty$. The only case, where this degeneration limit differs from the results of the previous subsection (cf. eqs. (3.11) and (3.12)) is $k=1$ and $r=0$ :

$$
\lim _{\tau \rightarrow i \infty} f^{(1)}(z-s, \tau) \mathrm{d} z=-\frac{1}{2} \frac{\mathrm{d} w}{w}+\frac{\mathrm{d} w}{w-e^{2 \pi i s}} .
$$

Note the occurrence of the root of unity $e^{2 \pi i s}$. Denoting the set of $N^{\text {th }}$ roots of unity by

$$
\mu_{N}=\left\{e^{2 \pi i s} \mid s \in \Lambda_{N}\right\}
$$

the generalization of eq. (3.13) to twists $b \in \Lambda_{N}+\Lambda_{N} \tau$ reads

$$
\lim _{\tau \rightarrow i \infty}-\mathrm{d} z \sum_{k=0}^{\infty} \sum_{b \in \Lambda_{N}+\Lambda_{N} \tau} f^{(k)}(z-b, \tau) \operatorname{ad}_{x_{b}}^{k}(y)=\tilde{y}_{N} \frac{\mathrm{d} w}{w}+\sum_{\zeta \in \mu_{N}} t_{\zeta} \frac{\mathrm{d} w}{w-\zeta},
$$

where ${ }^{7}$

$$
\begin{aligned}
\tilde{y}_{N} & =-\sum_{b \in \Lambda_{N}} \frac{\operatorname{ad}_{x_{b}}}{e^{2 \pi i \operatorname{ad}_{x_{b}}-1}}(y)+\sum_{b \in\left(\Lambda_{N}+\Lambda_{N} \tau\right) \backslash \Lambda_{N}} \frac{\operatorname{ad}_{x_{b}} e^{-2 \pi i r a d_{x_{b}}}}{e^{-2 \pi i \mathrm{ad}_{x_{b}}-1}}(y) \\
t_{\zeta} & =\left[y, x_{s}\right], \quad \text { for } \zeta=e^{2 \pi i s} \in \mu_{N} .
\end{aligned}
$$

Equation (3.26) is the generalization of eq. (3.14) to arbitrary twists in the lattice $\Lambda_{N}+\Lambda_{N} \tau$, and can be proved along the lines of the previous subsection. In particular, the expression for $\tilde{y}_{N}$ follows by repeating the steps which have been detailed around eq. (3.15).

\subsubsection{Deforming the integration contour}

Now the image of the integration contour $[0,1]_{\varepsilon}$ under the transformation $z \mapsto w=e^{2 \pi i z}$ is the unit circle around 0 , which is dented at the roots of unity $e^{2 \pi i s} \in \mu_{N}$ as pictured in

\footnotetext{
${ }^{7}$ Note that the definition of $\tilde{y}_{N}$ for all twists is different from eq. (3.14) in the previous subsection which is valid for generic twists only.
} 
figure 5 below. However, the point $w=1$ is special and will be taken care of by the regularization of subsection 3.2. Similar to the situation above, the dented unit circle is homotopic to $\left[P_{1}^{-1} C_{0}^{2 \pi}(\varepsilon) P_{1} \hat{C}_{\pi}^{0}(\varepsilon)\right]$ as depicted in figure 5 for twists in $\Lambda_{3}$.
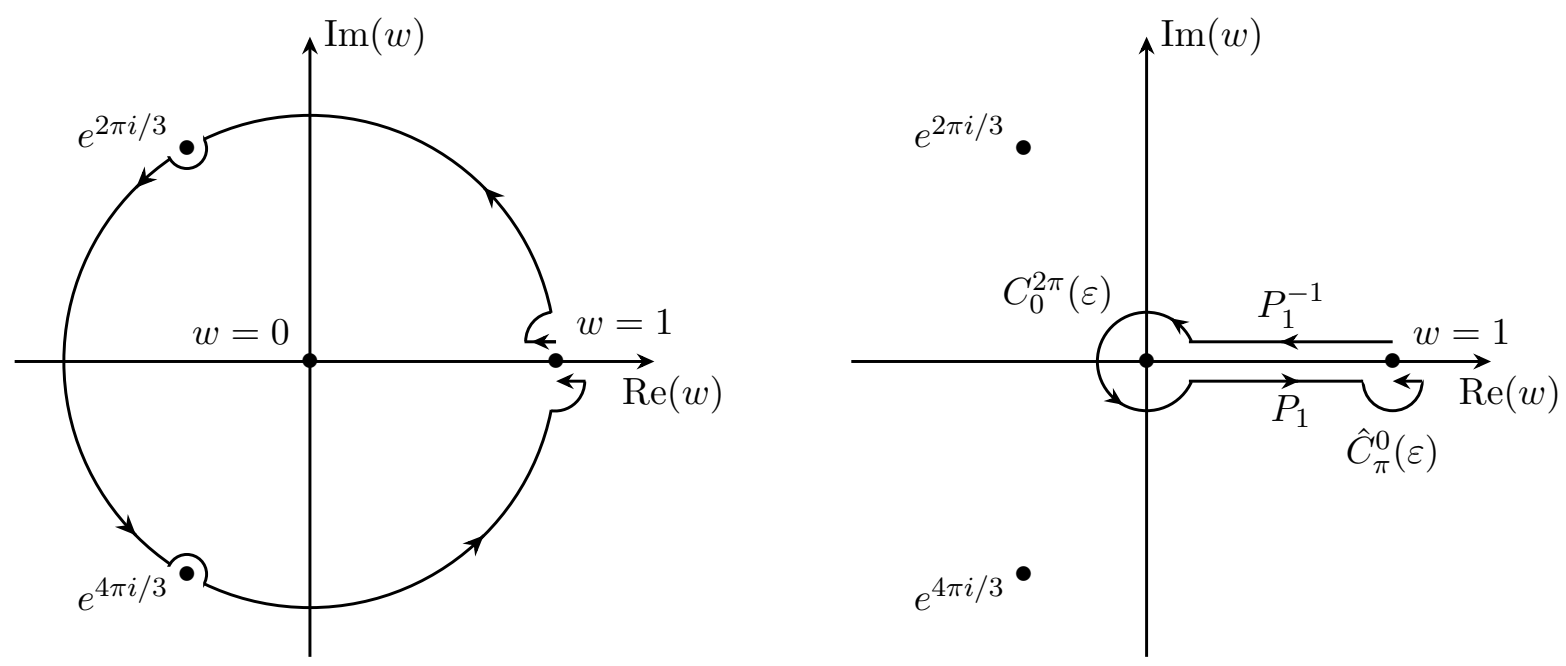

Figure 5: Deformation of the dented unit circle to the path composition $P_{1}^{-1} C_{0}^{2 \pi}(\varepsilon) P_{1} \hat{C}_{\pi}^{0}(\varepsilon)$. Proper rational twists $s=0, \frac{1}{N}, \ldots, \frac{N-1}{N}$ are mapped to unit roots $\zeta=e^{2 \pi i s} \in \mu_{N}$.

Hence, eq. (3.18) can be generalized to

$$
\lim _{\tau \rightarrow i \infty} A_{\Lambda_{N}+\Lambda_{N} \tau}^{\mathrm{twist}}(\tau)=e^{i \pi t_{1}} \Phi_{N}\left(\tilde{y}_{N},\left(t_{\zeta}\right)_{\zeta \in \mu_{N}}\right) e^{2 \pi i \tilde{y}_{N}} \Phi_{N}\left(\tilde{y}_{N},\left(t_{\zeta}\right)_{\zeta \in \mu_{N}}\right)^{-1}
$$

where $\Phi_{N}\left(e_{0},\left(e_{\zeta}\right)_{\zeta \in \mu_{N}}\right)$ is the cyclotomic version of the Drinfeld associator [42], defined by

$$
\Phi_{N}\left(e_{0},\left(e_{\zeta}\right)_{\zeta \in \mu_{N}}\right)=\tilde{\mathcal{P}} \exp \left(\int_{0}^{1}\left[\frac{e_{0}}{w}+\sum_{\zeta \in \mu_{N}} \frac{e_{\zeta}}{w-\zeta}\right] \mathrm{d} w\right)
$$

Since $\Phi_{N}$ is the generating series of $N$-cyclotomic MZVs $[3,13-15]$, the constant terms of teMZVs for arbitrary twists in the lattice $\Lambda_{N}+\Lambda_{N} \tau$ are $\mathbb{Q}\left[(2 \pi i)^{-1}\right]$-linear combinations of cyclotomic MZVs. Definitions and properties of cyclotomic MZVs are collected in appendix D, and examples for constant terms of teMZVs with proper rational twists can be found in appendix G.2.

As exemplified by $\omega_{0}\left(\begin{array}{c}1 \\ 1 / 2\end{array}\right)=-i \pi$, it is the regularization of divergences occurring for proper rational twists, which spoils the validity of the reflection property eq. (2.14) for letters $B=\frac{1}{b}$ with $b \in \Lambda_{N}^{\times}$. It would be interesting to identify an alternative regularization scheme where eq. $(2.14)$ is preserved.

\section{One-loop open-string amplitude}

This section is devoted to the discussion of the appearance of teMZVs in a physics context - in the low-energy expansion of scattering amplitudes in string theory [43-47. In general, string amplitudes at lower loop orders ${ }^{8} g \leq 2$, possibly also at $g=3,4$, can be represented by integrals over the moduli space of punctured Riemann surfaces of genus $g$. For one-loop scattering of

\footnotetext{
${ }^{8}$ In the Ramond-Neveu-Schwarz approach to superstring theory, $(g \geq 5)$-loop amplitudes cannot be derived from the moduli space of ordinary Riemann surfaces since the moduli space of the required super Riemann surfaces of genus $g \geq 5$ is not split 48 .
} 

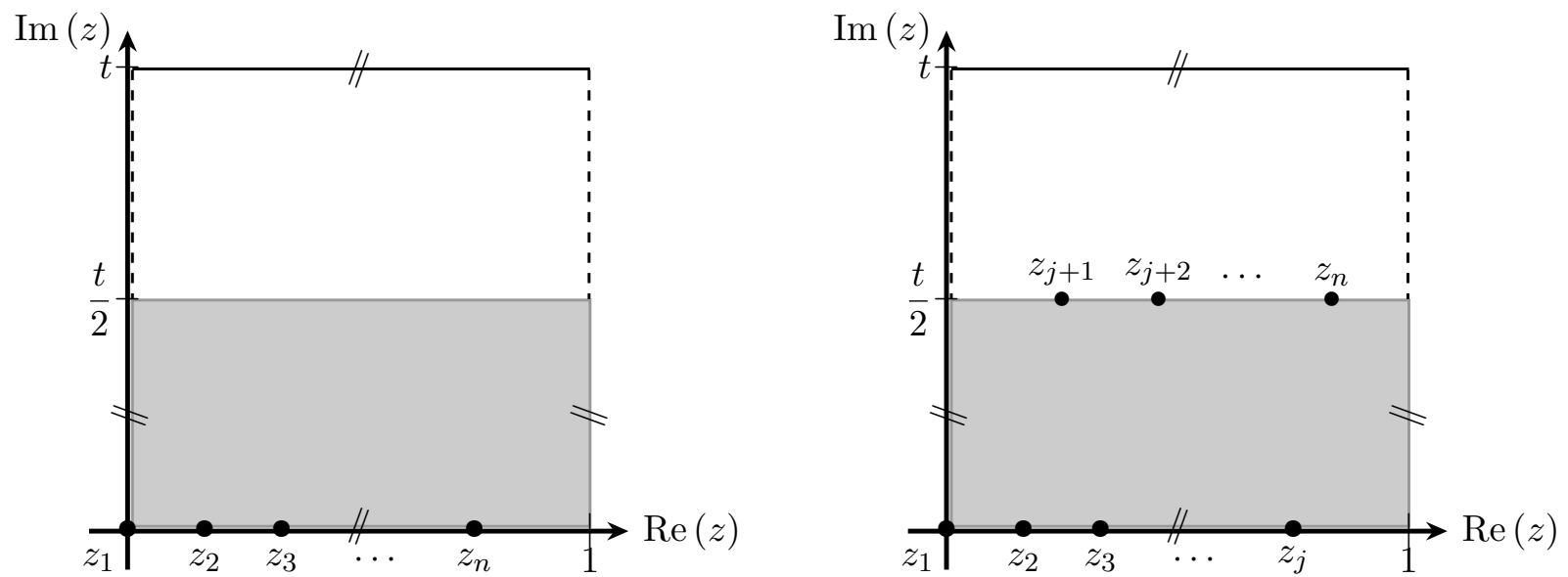

Figure 6: Worldsheets of cylinder topology are mapped to the shaded region, see the left and the right panel for the planar and the non-planar case, respectively. The punctures on the boundaries are taken to have coordinates with $\operatorname{Re}\left(z_{j}\right) \in[0,1]$ and either $\operatorname{Im}\left(z_{j}\right)=0$ or $\operatorname{Im}\left(z_{j}\right)=\frac{t}{2}$. The identification of edges is marked by $\approx$ and $/ /$, respectively, and inherited from a torus with modular parameter $\tau=i t$. The Mœbius topology is not drawn here, because all its contributions to the amplitude can be inferred from the planar cylinder topology. This can be seen by the change of variables described in ref. [49].

open strings, the Riemann surfaces or worldsheets of interest are the cylinder and the Mobius strip. The punctures - the insertion points of vertex operators for external states - are then integrated over the boundary components of the worldsheets. These boundary integrals are weighted by traces over Lie-algebra generators $t^{a}$ associated with the gauge degrees of freedom of the open-string states: Each boundary component contributes a separate trace factor, in each of which the order of multiplication matches the ordering of the associated punctures.

A convenient parametrization of the one-loop open-string topologies - the cylinder and the Møbius strip - can be obtained starting from the torus by restricting the modular parameter to $\tau_{C}=i t$ and to $\tau_{M}=i t+\frac{1}{2}$, respectively, where $t \in \mathbb{R}_{+}$. In both cases, the boundary is parametrized via $z \in \mathbb{C} /(\mathbb{Z}+\mathbb{Z} \tau)$ with $\operatorname{Re}(z) \in[0,1]$ and $\operatorname{Im}(z)=0$ or $\operatorname{Im}(z)=\frac{t}{2}$ which is sometimes referred to as the closed-string channel.

In this setup, elliptic iterated integrals eq. (2.1) appear naturally when integrating over moduli spaces of cylinder- and Mœbius-strip punctures. This is yet another example, where the iterated integrals on the boundary of open-string worldsheets yield special values of polylogarithms tailored to the corresponding Riemann surfaces, generalizing the ubiquity of MZVs at genus zero.

Cylindrical worldsheets with all insertions on the same boundary are referred to as planar cylinders. For these contributions to the amplitude, all integrals over the punctures were shown to boil down to eMZVs in 30 . Moreover, the only difference between integrals over punctures on the Mœbius strip and those on the planar cylinder is the value of the modular parameter $\tau$ [49: therefore one can straightforwardly convert the contributions from the planar cylinder to those of the Mœbius strip by

- replacing $q_{C}=e^{2 \pi i \tau_{C}}=e^{-2 \pi t}$ in the Fourier expansion of the eMZVs in the planar-cylinder contribution by $q_{M}=e^{2 \pi i \tau_{M}}=e^{-2 \pi t+i \pi}=-q_{C}$; this results in alternating relative signs between the coefficients in the $q_{C}$-expansion of the Mobius-strip contributions and the cylinder contributions.

- inserting a factor of $\pm \frac{32}{N_{G}}$ for the Mœbius strip to account for its single boundary of doubled 
length compared to individual boundary components of the cylinder $[49$. The "+" sign is for gauge groups $U S p\left(N_{G}\right)$ and "-" for $S O\left(N_{G}\right)$.

Hence, for a gauge group $S O(32)$, the constant term in the $q$-expansion w.r.t. $q_{C}$ which would give rise to a UV divergence upon integration over $t \in \mathbb{R}_{+}$cancels between the cylinder and the Mœbius strip [49].

The double-trace contributions, on the other hand, stem entirely from the cylinder topology with punctures on two different boundaries - non-planar cylinder diagrams. We will see that the integrals over the punctures boil down to teMZVs with purely imaginary modular parameter and twists $b \in\left\{0, \frac{\tau_{C}}{2}\right\}=\left\{0, \frac{i t}{2}\right\}$.

In the planar case mentioned above the link between eMZVs and the worldsheet integral over the cylinder boundary was established as follows: Punctures on the same boundary enter through the genus-one Green function at real arguments which can be written as an integral over the weighting function $f^{(1)}(x)$ with real argument $x \in(0,1)|30|$. Consequently, we will show that the new ingredient in the case of the non-planar cylinder, i.e. the genus-one Green function for two insertions on different boundaries, is related to integrals over $f^{(1)}(x-\tau / 2)$. Hence, the non-planar contributions may be expressed as iterated integrals on $E_{\tau}^{\times} \backslash\{\tau / 2\}$ described above, which eventually lead to the teMZVs introduced in section 2 .

In order to simplify the final formulas, we employ the differential equation of section 3 to build in all the relations among the teMZVs we encounter (see appendix G.3). In fact, as will be detailed in section 4.3, these relations ultimately reduce all instances of teMZVs to eMZVs alone. Still, teMZVs are an essential tool for intermediate steps and to render the subsequent computations completely algorithmic.

\subsection{The four-point integrals}

We will illustrate the emergence of teMZVs through the non-planar contribution to the fourpoint one-loop amplitude of the open superstring. Its dependence on the external polarizations enters through a prefactor $K$ universal to all worldsheet topologies 50$]$ and is irrelevant for the subsequent discussion. Then, setting $q_{C}=q$ and $q_{M}=-q$ as discussed above, the complete expression for the one-loop open-string four-point amplitude reads [49]

$$
\begin{aligned}
\mathcal{A}_{4}^{1-\text { loop }}=K \int_{0}^{1} \frac{\mathrm{d} q}{q}\{ & \operatorname{Tr}\left(t^{1} t^{2} t^{3} t^{4}\right)\left[N_{G} I_{1234}(q)-32 I_{1234}(-q)\right] \\
& \left.+\operatorname{Tr}\left(t^{1} t^{2}\right) \operatorname{Tr}\left(t^{3} t^{4}\right) I_{12 \mid 34}(q)+\operatorname{cyc}(2,3,4)\right\},
\end{aligned}
$$

where $t^{a}$ are traceless generators of the gauge group $S O\left(N_{G}\right)$ and the traces are taken in its fundamental representation. The accompanying integrals are given by ${ }^{9}$

$$
\begin{aligned}
I_{1234}(q)= & \int_{0}^{1} \mathrm{~d} x_{4} \int_{0}^{x_{4}} \mathrm{~d} x_{3} \int_{0}^{x_{3}} \mathrm{~d} x_{2} \int_{0}^{x_{2}} \mathrm{~d} x_{1} \delta\left(x_{1}\right) \prod_{i<j}^{4} \exp \left[\frac{1}{2} s_{i j} G\left(x_{i j}, \tau\right)\right], \\
I_{12 \mid 34}(q)= & \int_{0}^{1} \mathrm{~d} x_{4} \int_{0}^{1} \mathrm{~d} x_{3} \int_{0}^{1} \mathrm{~d} x_{2} \int_{0}^{1} \mathrm{~d} x_{1} \delta\left(x_{1}\right) \\
& \times \exp \left[\frac{1}{2} s_{12} G\left(x_{12}, \tau\right)+\frac{1}{2} s_{34} G\left(x_{34}, \tau\right)+\frac{1}{2} \sum_{\substack{i=1,2 \\
j=3,4}} s_{i j} G\left(x_{i j}-\tau / 2, \tau\right)\right],
\end{aligned}
$$

\footnotetext{
${ }^{9}$ Note that $I_{1234}(q)$ was denoted by $I_{4 \mathrm{pt}}(1,2,3,4)$ in ref. 30 and that $I_{12 \mid 34}(q)$ is defined with a factor of two in comparison to the integral $h(s, u)$ in ref. 31 because we do not impose $x_{3}<x_{4}$ as done in the latter reference.
} 
where the insertion points $z_{1,2}=x_{1,2}$ and $z_{3,4}=x_{3,4}+\tau / 2$ of the vertex operators are parametrized by real integration variables $x_{i}$ with $x_{i j}=x_{i}-x_{j}$, see figure 6 . Translation invariance on a genusone surface has been used to fix $x_{1}=0$ through the above delta function. The genus-one Green functions ${ }^{10} 32$

$$
G(z, \tau)=\log \left|\frac{\theta_{1}(z, \tau)}{\theta_{1}^{\prime}(0, \tau)}\right|^{2}-\frac{2 \pi}{\operatorname{Im}(\tau)} \operatorname{Im}(z)^{2}
$$

depend on the differences of punctures $z_{i}$ and their second argument $\tau$ will often be suppressed. Considering the parametrization of the cylinder visualized in figure 6, vertex insertions on different boundaries give rise to arguments $x_{i j}-\tau / 2$ as for instance seen in the contribution $G\left(x_{13}-\tau / 2, \tau\right)$ to the exponent of eq. (4.3).

Given the relations between the dimensionless Mandelstam invariants ${ }^{11}$,

$$
s_{34}=s_{12}, \quad s_{14}=s_{23}, \quad s_{13}=s_{24}=-s_{12}-s_{23},
$$

the integrands of eqs. $(\overline{4.2})$ and $(\overline{4.3})$ are unchanged if the Green function eq. $(\overline{4.4})$ is shifted by a $z$-independent function. This feature will be made use of in the following subsections.

Configurations with three punctures on the same boundary lead to color factors such as $\operatorname{Tr}\left(t^{1} t^{2} t^{3}\right) \operatorname{Tr}\left(t^{4}\right)$ which vanish for traceless $S O\left(N_{G}\right)$ generators considered in eq. (4.1). Nevertheless, the accompanying integral

$$
\begin{aligned}
I_{123 \mid 4}(q)= & \int_{0}^{1} \mathrm{~d} x_{4} \int_{0}^{1} \mathrm{~d} x_{3} \int_{0}^{x_{3}} \mathrm{~d} x_{2} \int_{0}^{x_{2}} \mathrm{~d} x_{1} \delta\left(x_{1}\right) \\
& \left.\times \exp \left[\frac{1}{2} \sum_{1 \leq i<j}^{3} s_{i j} G\left(x_{i j}, \tau\right)+\frac{1}{2} \sum_{j=1}^{3} s_{j 4} G\left(x_{j 4}-\tau / 2, \tau\right)\right]\right]
\end{aligned}
$$

plays an important rôle for one-loop monodromy relations [53,31]. It will be demonstrated in appendix $\mathrm{H}$ that eq. (4.6) may be expanded in terms of teMZVs using the same techniques as will be applied to the integral $I_{12 \mid 34}(q)$ in eq. (4.3) along with two punctures on each boundary. For both non-planar integrals $I_{12 \mid 34}(q)$ and $I_{123 \mid 4}(q)$, our results up to and including the order of $s_{i j}^{3}$ can be simplified to ultimately yield combinations of eMZVs, i.e., as mentioned earlier, all of their twisted counterparts are found to drop out at the orders considered.

\subsubsection{Analytic versus non-analytic momentum dependence}

The one-loop four-point amplitude is a non-analytic function of the Mandelstam invariants eq. (4.5): From the integration over $q$ in eq. (4.1), the region with $t \rightarrow \infty$ or $q=e^{-2 \pi t} \rightarrow 0$ leads to branch cuts well-known from the Feynman integrals in the field-theory limit $[50$. Moreover, the non-planar contribution $I_{12 \mid 34}(q)$ additionally integrates to kinematic poles in $s_{12}$, reflecting the exchange of closed-string states between the cylinder boundaries [44. Since both the poles and the branch cuts stem from the integration over $q$, the integrals eqs. (4.2) and (4.3) over the punctures by themselves do not reflect the singularity structure of the one-loop amplitude.

\footnotetext{
${ }^{10}$ As pointed out earlier, the cylinder and the Mœbius-strip worldsheets for open-string one-loop amplitudes are derived from a torus, and the restrictions of the modular parameters and the punctures can be understood in terms of involutions. The open-string Green function eq. (4.4) is constructed using the method of images and therefore takes the same functional form as its closed-string counterpart adapted to the torus: This follows from the localization of the open-string punctures on the boundaries of the cylinder and the Moebius-strip worldsheets which are in turn fixed points of the defining involutions 51,52 .

${ }^{11}$ Mandelstam invariants are defined by $s_{i j}=\alpha^{\prime}\left(k_{i}+k_{j}\right)^{2}$, where $k_{i}$ denote the momenta of the external open-string states with $i=1,2,3,4$ subject to momentum conservation $\sum_{i=1}^{4} k_{i}=0$ and $k_{i}^{2}=0$.
} 
At fixed values of $q$ it is possible to separate the overall amplitude into analytic and nonanalytic parts. For the four-point closed-string one-loop amplitude, a careful procedure to isolate the logarithmic dependence on $s_{i j}$ has been developed in ref. [54]: this method allows for a focused study of the analytic sector where modular graph functions take the rôle of eMZVs 55,56 .

While the integrals in the expansion of eq. (4.2) have been performed at fixed value of $q$, integrating for instance eq. (4.7) below and its counterpart from the Mœbius strip over $q$ introduces divergences, also for the gauge group $S O(32)$. The choice of regularization scheme for these divergences (see 31 for an example at the first subleading order in $\alpha^{\prime}$ ) reflects a particular way of splitting the analytic and non-analytic parts of the final expression for the amplitude after integrating over $q$.

\subsubsection{The low-energy expansion in the single-trace sector}

In the following, we will study the analytic part of the non-planar one-loop four-point amplitude by Taylor-expansion of eq. (4.3) in $s_{i j}$ and thereby in $\alpha^{\prime}$, probing the low-energy behaviour. The analogous low-energy expansion for the single-trace integral eq. $(4.2)$ has been performed in $[30$,

$$
\begin{aligned}
& I_{1234}(q)=\omega(0,0,0)-2 \omega(0,1,0,0)\left(s_{12}+s_{23}\right)+2 \omega(0,1,1,0,0)\left(s_{12}^{2}+s_{23}^{2}\right) \\
& -2 \omega(0,1,0,1,0) s_{12} s_{23}+\beta_{5}\left(s_{12}^{3}+2 s_{12}^{2} s_{23}+2 s_{12} s_{23}^{2}+s_{23}^{3}\right)+\beta_{2,3} s_{12} s_{23}\left(s_{12}+s_{23}\right)+\mathcal{O}\left(\alpha^{\prime 4}\right)
\end{aligned}
$$

with the following combinations of eMZVs at order $\alpha^{\prime 3}$ :

$$
\begin{aligned}
\beta_{5} & =\frac{4}{3}\left[\omega(0,0,1,0,0,2)+\omega(0,1,1,0,1,0)-\omega(2,0,1,0,0,0)-\zeta_{2} \omega(0,1,0,0)\right] \\
\beta_{2,3} & =\frac{\zeta_{3}}{12}+\frac{8 \zeta_{2}}{3} \omega(0,1,0,0)-\frac{5}{18} \omega(0,3,0,0) .
\end{aligned}
$$

It was explained in the reference that the dependence of the single-trace integral eq. (4.7) on $q$ is captured by eMZVs at any order in $\alpha^{\prime}$. Note that the contributions of the planar cylinder and the Mœbius strip to eq. (4.1) are obtained by integrating eq. (4.7) over arguments $q \rightarrow e^{-2 \pi t}$ and $q \rightarrow-e^{-2 \pi t}$, respectively, with $t \in \mathbb{R}_{+}$.

In analogy with eq. (4.7), we will determine the $\alpha^{\prime}$-expansion of the non-planar integral eq. (4.3) in the framework of teMZVs. Since the main emphasis of this article is to exemplify the use of teMZVs in the calculation of non-planar one-loop amplitudes, a detailed analysis of the singularity structure after integration over $q$ is left for the future.

\subsection{The genus-one Green function as an elliptic iterated integral}

The link between open-string amplitudes and the framework of elliptic iterated integrals is the holomorphic derivative ${ }^{12}$

$$
\partial_{z} G(z, \tau)=f^{(1)}(z, \tau), \quad z \in \mathbb{C} \backslash(\mathbb{Z}+\mathbb{Z} \tau),
$$

\footnotetext{
${ }^{12}$ In contrast to section 3 , where $\partial_{z}$ denoted the derivative w.r.t. the real parameter $z, \partial_{z}$ denotes the holomorphic derivative in this section.
} 
of the genus-one Green function eq. (4.4) in the integrals eqs. $(4.2)$ and $(4.3)$. It allows to rewrite the exponent in the non-planar integral eq. (4.3) into the form

$$
\begin{gathered}
\frac{1}{2} s_{12} G\left(x_{12}, \tau\right)+\frac{1}{2} s_{34} G\left(x_{34}, \tau\right)+\sum_{\substack{i=1,2 \\
j=3,4}} \frac{1}{2} s_{i j} G\left(x_{i j}-\tau / 2, \tau\right) \\
=s_{12} P\left(x_{12}\right)+s_{34} P\left(x_{34}\right)+\sum_{\substack{i=1,2 \\
j=3,4}} s_{i j} Q\left(x_{i j}\right)
\end{gathered}
$$

where the entire dependence on the real parts ${ }^{13} x_{i} \in \mathbb{R}$ of the punctures $z_{1,2}=x_{1,2}$ and $z_{3,4}=$ $x_{3,4}+\tau / 2$ is captured via elliptic iterated integrals eq. (2.1):

$$
\begin{aligned}
& P(x)=\int_{0}^{x} \mathrm{~d} y f^{(1)}(y)=\Gamma\left(\begin{array}{c}
1 \\
0
\end{array} ; x\right) \\
& Q(x)=c(q)+\int_{0}^{x} \mathrm{~d} y f^{(1)}(y-\tau / 2)=c(q)+\Gamma\left(\begin{array}{c}
1 \\
\tau / 2
\end{array} ; x\right) .
\end{aligned}
$$

The appearance of the $x_{i}$-independent quantity

$$
c(q)=-\frac{i \pi}{2}-\frac{1}{8} \log (q)+2 \sum_{m=1}^{\infty} \frac{1}{m}\left[\frac{q^{m}}{1-q^{m}}-\frac{q^{m / 2}}{1-q^{m}}\right]
$$

is special to the non-planar cylinder and caused by the different properties of the Green functions $G\left(x_{i j}\right)$ and $G\left(x_{i j}-\tau / 2\right)$ connecting punctures on the same and different boundaries of the cylinder, respectively.

In passing to the right hand side of eq. (4.11) we have used momentum conservation $\sum_{i<j}^{4} s_{i j}=$ 0 to discard

$$
\frac{1}{2} G(x)-P(x)=\frac{i \pi}{2}-\log (2 \pi)=\frac{1}{2} G(x-\tau / 2)-Q(x) .
$$

A detailed explanation of the relations above and the underlying regularization will be given in the following two subsections.

\subsection{1 $G(x)$ versus $P(x)$}

In the case of punctures on the same boundary of the cylinder, the Green functions with arguments $x \in[0,1]$ and $\tau \in i \mathbb{R}_{+}$reduce to $\frac{1}{2} G(x, \tau)=\log \frac{\theta_{1}(x, \tau)}{\theta_{1}^{\prime}(0, \tau)}$. Up to an additive constant, this expression can be recovered by the following integration with a regulator $\varepsilon>0$ in the lower limit:

$$
\begin{aligned}
\int_{\varepsilon}^{x} \mathrm{~d} y f^{(1)}(y) & =\log \left(\theta_{1}(x)\right)-\log \left(\theta_{1}(\varepsilon)\right) \\
& =\log \left(\theta_{1}(x)\right)-\log \left(\theta_{1}^{\prime}(0)\right)-\log (\varepsilon)+\mathcal{O}(\varepsilon) \\
& =\frac{1}{2} G(x)-\log (-2 \pi i \varepsilon)+\log (-2 \pi i)+\mathcal{O}(\varepsilon) .
\end{aligned}
$$

The regularization scheme for the limit $\varepsilon \rightarrow 0$ has to be chosen consistently with the treatment of divergent eMZVs: Following the conventions of $[30]$, the regularized value of an eMZV is

\footnotetext{
${ }^{13}$ Restricting the first argument $G(x, \tau)$ to be real (as appropriate for our parametrization of the cylinder) leads to a relative factor of two between $\partial_{x} G(x, \tau)=2 f^{(1)}(x, \tau), x \in \mathbb{R}$ and eq. (4.10). This factor of two has been neglected in early versions of 30 , and it is not altered by a complex shift $\partial_{x} G(x-\tau / 2, \tau)=2 f^{(1)}(x-\tau / 2, \tau), x \in \mathbb{R}$.
} 
defined to be the constant term in an expansion ${ }^{14}$ w.r.t. $\log (-2 \pi i \varepsilon)$, and we choose the principal branch of the $\operatorname{logarithm}$, such that $\log (-i)=-\frac{i \pi}{2}$. Hence, $\log (-2 \pi i \varepsilon)$ is formally set to zero in eq. (4.16), and we obtain

$$
P(x)=\lim _{\varepsilon \rightarrow 0} \operatorname{Reg} \int_{\varepsilon}^{x} \mathrm{~d} y f^{(1)}(y)=\frac{1}{2} G(x)+\log (-2 \pi i)=\frac{1}{2} G(x)-\frac{i \pi}{2}+\log (2 \pi),
$$

reproducing the first equality in eq. (4.15).

\subsection{2 $G(x-\tau / 2)$ versus $Q(x)$}

Pairs of punctures on different boundaries of the cylinder lead to arguments $x-\tau / 2$ of the Green function eq. (4.4), where $x \in[0,1]$. In these cases, the Green function may be related to the integral

$$
\begin{aligned}
\int_{0}^{x} \mathrm{~d} y f^{(1)}(y-\tau / 2) & =\log \left(\theta_{1}(x-\tau / 2)\right)-i \pi x-\log \left(\theta_{1}(-\tau / 2)\right) \\
& =\log \left|\theta_{1}(x-\tau / 2)\right|-\frac{i \pi}{2}-\log \left(-\theta_{1}(\tau / 2)\right) \\
& =\frac{1}{2} G(x-\tau / 2)+\log \theta_{1}^{\prime}(0)-\frac{1}{8} \log (q)-\frac{i \pi}{2}-\log \left(-i q^{-1 / 8} \theta_{4}(0)\right) \\
& =\frac{1}{2} G(x-\tau / 2)+\log \left(\frac{\theta_{1}^{\prime}(0)}{\theta_{4}(0)}\right)=\frac{1}{2} G(x-\tau / 2)-\frac{1}{2} G(\tau / 2),
\end{aligned}
$$

without any need for regularization. In passing to the second line, we have chosen the principal branch of the logarithm to relate $\log \left(\theta_{1}(x-\tau / 2)\right)-i \pi x=\log \left|\theta_{1}(x-\tau / 2)\right|-\frac{i \pi}{2}$, see appendix A for our conventions and several identities for the theta functions. In the next step, we have introduced the Green function via $\log \left|\theta_{1}(x-\tau / 2)\right|=\frac{1}{2} G(x-\tau / 2)+\log \theta_{1}^{\prime}(0)-\frac{1}{8} \log (q)$ and used the theta-function identity $\theta_{1}(\tau / 2)=i q^{-1 / 8} \theta_{4}(0)$, cf. eq. (A.3).

Using the infinite-product representations eq. (A.1) of the theta functions, the result of eq. (4.18) can be rewritten as 57

$$
\begin{aligned}
& \int_{0}^{x} \mathrm{~d} y f^{(1)}(y-\tau / 2)-\frac{1}{2} G(x-\tau / 2)=\log \left(\frac{\theta_{1}^{\prime}(0)}{\theta_{4}(0)}\right) \\
& =\log \left(2 \pi q^{1 / 8}\right)+2 \sum_{n=1}^{\infty}\left[\log \left(1-q^{n}\right)-\log \left(1-q^{n-1 / 2}\right)\right] \\
& =\log \left(2 \pi q^{1 / 8}\right)+2 \sum_{m=1}^{\infty} \frac{1}{m}\left[\frac{q^{m / 2}}{1-q^{m}}-\frac{q^{m}}{1-q^{m}}\right] \\
& =-\frac{i \pi}{2}+\log (2 \pi)-c(q)
\end{aligned}
$$

In passing to the third line, we have rearranged the infinite sums ${ }^{15}$ to identify the quantity $c(q)$

\footnotetext{
${ }^{14}$ The expansion in $\log (-2 \pi i \varepsilon)$ will ensure that the constant terms of eMZVs are $\mathbb{Q}\left[(2 \pi i)^{-1}\right]$-linear combinations of MZVs, as opposed to $\mathbb{Q}\left[(2 \pi i)^{-1}, \log (2 \pi)\right]$-linear combinations of MZVs (as in 8, Proposition 2.8).

${ }^{15}$ Note the following useful intermediate expressions whose sum is denoted by $-\frac{1}{2} Q_{3}$ in ref. 31 .

$$
\sum_{n=1}^{\infty} \log \left(1-q^{n}\right)=-\sum_{m, n=1}^{\infty} \frac{q^{m n}}{m}=-\sum_{m=1}^{\infty} \frac{1}{m} \frac{q^{m}}{1-q^{m}}, \quad-\sum_{n=1}^{\infty} \log \left(1-q^{n-\frac{1}{2}}\right)=\sum_{m, n=1}^{\infty} \frac{q^{m n-m / 2}}{m}=\sum_{m=1}^{\infty} \frac{1}{m} \frac{q^{m / 2}}{1-q^{m}} .
$$
}


in eq. (4.14). In this way, one arrives at

$$
Q(x)=c(q)+\int_{0}^{x} \mathrm{~d} y f^{(1)}(y-\tau / 2)=\frac{1}{2} G(x-\tau / 2)-\frac{i \pi}{2}+\log (2 \pi),
$$

and the second equality in eq. $(4.15)$ is confirmed.

\subsection{Non-planar contribution to the four-point amplitude}

Using the identities discussed in the previous subsections, the integral eq. (4.3) relevant to the non-planar cylinder can be written as

$$
I_{12 \mid 34}(q)=\int_{12}^{34} \exp \left[s_{12} P\left(x_{12}\right)+s_{34} P\left(x_{34}\right)+\sum_{\substack{i=1,2 \\ j=3,4}} s_{i j} Q\left(x_{i j}\right)\right]
$$

where $P(x)$ and $Q(x)$ are given by the elliptic iterated integrals eqs. $(4.12)$ and $(4.13)$ and we have introduced the following shorthand for the integration measure:

$$
\int_{12}^{34}=\int_{0}^{1} \mathrm{~d} x_{4} \int_{0}^{1} \mathrm{~d} x_{3} \int_{0}^{1} \mathrm{~d} x_{2} \int_{0}^{1} \mathrm{~d} x_{1} \delta\left(x_{1}\right) .
$$

We will now investigate the Taylor-expansion of eq. (4.21) in the dimensionless Mandelstam invariants eq. (4.5) and thus in $\alpha^{\prime}$ by expanding the exponentials $e^{s_{i j} P\left(x_{i j}\right)}$ and $e^{s_{i j} Q\left(x_{i j}\right)}$ in the integrand:

$$
I_{12 \mid 34}(q)=e^{-2 s_{12} c(q)} \int_{12}^{34} \sum_{n_{i j}=0}^{\infty} \frac{\left(s_{12} \Gamma\left(\begin{array}{l}
1 \\
0
\end{array} ; x_{12}\right)\right)^{n_{12}}\left(s_{34} \Gamma\left(\begin{array}{c}
1 \\
0
\end{array} ; x_{34}\right)\right)^{n_{34}}}{n_{12} ! n_{34} !} \prod_{\substack{i=1,2 \\
j=3,4}} \frac{\left(s_{i j} \Gamma\left(\begin{array}{c}
1 \\
\tau / 2
\end{array} ; x_{i j}\right)\right)^{n_{i j}}}{n_{i j} !}
$$

The punctures $x_{i}$ only enter via elliptic iterated integrals, and Fay relations among the weighting functions $f^{(n)}$ guarantee that the individual integrations over $x_{i}$ can always be performed in terms of further elliptic iterated integrals, see appendix I. Hence, each order in $\alpha^{\prime}$ can be expressed in terms of teMZVs and the quantity $c(q)$ in eq. (4.14), where the latter will also be related to teMZVs in eq. (4.49). Moreover, the explicit results up to the third order can in fact be expressed in terms of (untwisted) eMZVs only, without the need to involve their twisted counterparts. Whether this behaviour persists at any order in $\alpha^{\prime}$ will be discussed in subsection 4.3.5.

\subsubsection{Structure of the leading orders $\alpha^{\prime \leq 3}$}

As a first step towards an expansion in terms of teMZVs, we classify the inequivalent integrals w.r.t. the cycle structure of $\operatorname{Tr}\left(t^{1} t^{2}\right) \operatorname{Tr}\left(t^{3} t^{4}\right)$ which occur at the orders $\alpha^{\prime \leq 3}$ of eq. (4.23): We will use the shorthand $P_{i j}=P\left(x_{i j}\right)$ and $Q_{i j}=Q\left(x_{i j}\right)$ for the two integrals at order $\alpha^{1}$,

$$
d_{1}^{1}=\int_{12}^{34} P_{12}, \quad d_{2}^{1}=\int_{12}^{34} Q_{13}
$$

the six integrals at order $\alpha^{\prime 2}$,

$$
d_{1}^{2}=\frac{1}{2} \int_{12}^{34} P_{12}^{2}, \quad d_{3}^{2}=\int_{12}^{34} P_{12} Q_{13}, \quad d_{5}^{2}=\int_{12}^{34} Q_{13} Q_{14}
$$




$$
d_{2}^{2}=\frac{1}{2} \int_{12}^{34} Q_{13}^{2}, \quad d_{4}^{2}=\int_{12}^{34} P_{12} P_{34}, \quad d_{6}^{2}=\int_{12}^{34} Q_{13} Q_{24}
$$

and the twelve integrals at order $\alpha^{\prime 3}$ :

$$
\begin{aligned}
& d_{1}^{3}=\frac{1}{6} \int_{12}^{34} P_{12}^{3}, \quad d_{5}^{3}=\frac{1}{2} \int_{12}^{34} P_{12}^{2} P_{34}, \quad d_{9}^{3}=\int_{12}^{34} P_{12} Q_{13} Q_{24} \\
& d_{2}^{3}=\frac{1}{6} \int_{12}^{34} Q_{13}^{3}, \quad d_{6}^{3}=\frac{1}{2} \int_{12}^{34} Q_{13}^{2} Q_{14}, \quad d_{10}^{3}=\int_{12}^{34} P_{12} Q_{13} Q_{14} \\
& d_{3}^{3}=\frac{1}{2} \int_{12}^{34} P_{12}^{2} Q_{13}, \quad d_{7}^{3}=\frac{1}{2} \int_{12}^{34} Q_{13}^{2} Q_{24}, \quad d_{11}^{3}=\int_{12}^{34} P_{34} Q_{13} Q_{14} \\
& d_{4}^{3}=\frac{1}{2} \int_{12}^{34} P_{12} Q_{13}^{2}, \quad d_{8}^{3}=\int_{12}^{34} P_{12} P_{34} Q_{13}, \quad d_{12}^{3}=\int_{12}^{34} Q_{13} Q_{14} Q_{23} .
\end{aligned}
$$

In fact, some of the above $d_{i}^{j}$ can be related via cyclicity and reflection properties of the five-point open-string worldsheet setup where the integration measure eq. $(4.22)$ is generalized to

$$
\int_{123}^{45}=\int_{0}^{1} \mathrm{~d} x_{5} \int_{0}^{1} \mathrm{~d} x_{4} \int_{0}^{1} \mathrm{~d} x_{3} \int_{0}^{x_{3}} \mathrm{~d} x_{2} \int_{0}^{x_{2}} \mathrm{~d} x_{1} \delta\left(x_{1}\right)
$$

Using $\partial_{i} Q_{i j}=-\partial_{j} Q_{i j}$ and the vanishing of $\int_{0}^{1} \mathrm{~d} x_{j} \partial_{j} Q_{i j}$ by double periodicity of the Green function, we find one relation

$$
0=\int_{123}^{45} Q_{25} \partial Q_{14}=\int_{123}^{45} Q_{25}\left(Q_{24}-Q_{34}\right) \quad \Rightarrow \quad d_{5}^{2}=d_{6}^{2}
$$

among the integrals eq. (4.25) at order $\alpha^{\prime 2}$. The same methods yield the two relations

$$
\left.\begin{array}{l}
0=\int_{123}^{45} Q_{25}^{2} \partial Q_{14} \\
0=\int_{123}^{45} P_{23} Q_{25} \partial Q_{14}
\end{array}\right\} \Rightarrow\left\{\begin{array}{l}
d_{7}^{3}=d_{6}^{3} \\
d_{9}^{3}=d_{10}^{3}
\end{array}\right.
$$

among the integrals eq. (4.26) at order $\alpha^{\prime 3}$.

\subsection{2 teMZVs at orders $\alpha^{\prime \leq 3}$}

As a next step, we exploit the representations eqs. (4.12) and (4.13) of $P_{i j}$ and $Q_{i j}$ to express the above $d_{j}^{i}$ in terms of teMZVs: The two instances at order $\alpha^{\prime}$ yield

$$
\begin{aligned}
d_{1}^{1} & =\int_{0}^{1} \mathrm{~d} x_{2} \int_{0}^{x_{2}} \mathrm{~d} y f^{(1)}(y)=\omega\left(\begin{array}{l}
1,0 \\
0,0
\end{array}\right) \\
d_{2}^{1} & =c(q)+\int_{0}^{1} \mathrm{~d} x_{3} \int_{0}^{x_{3}} \mathrm{~d} y f^{(1)}(y-\tau / 2) \\
& =c(q)+\omega\left(\begin{array}{cc}
1, & 0 \\
\tau / 2, & 0
\end{array}\right)
\end{aligned}
$$

and we can similarly convert the five independent integrals at order $\alpha^{2}$ to

$$
\begin{aligned}
& d_{1}^{2}=\omega\left(\begin{array}{lll}
1, & 1, & 0 \\
0, & 0,0
\end{array}\right) \\
& d_{2}^{2}=\frac{1}{2} c(q)^{2}+c(q) \omega\left(\begin{array}{cc}
1, & 0 \\
\tau / 2, & 0
\end{array}\right)+\omega\left(\begin{array}{ccc}
1, & 1, & 0 \\
\tau / 2, & \tau / 2, & 0
\end{array}\right) \\
& d_{3}^{2}=d_{1}^{1} d_{2}^{1}=\omega\left(\begin{array}{ll}
1, & 0 \\
0, & 0
\end{array}\right)\left(c(q)+\omega\left(\begin{array}{cc}
1, & 0 \\
\tau / 2, & 0
\end{array}\right)\right)
\end{aligned}
$$




$$
\begin{aligned}
& d_{4}^{2}=\left(d_{1}^{1}\right)^{2}=\omega\left(\begin{array}{c}
1,0 \\
0,0
\end{array}\right)^{2} \\
& d_{5}^{2}=\left(d_{2}^{1}\right)^{2}=\left(c(q)+\omega\left(\begin{array}{cc}
1, & 0 \\
\tau / 2, & 0
\end{array}\right)\right)^{2}
\end{aligned}
$$

and the ten independent integrals at order $\alpha^{\prime 3}$ to

$$
\begin{aligned}
& d_{1}^{3}=\omega\left(\begin{array}{l}
1,1,1,0 \\
0,0,0,0
\end{array}\right) \\
& d_{2}^{3}=\frac{1}{6} c(q)^{3}+\frac{1}{2} c(q)^{2} \omega\left(\begin{array}{cc}
1, & 0 \\
\tau / 2, & 0
\end{array}\right)+c(q) \omega\left(\begin{array}{ccc}
1, & 1, & 0 \\
\tau / 2, & \tau / 2, & 0
\end{array}\right)+\omega\left(\begin{array}{cccc}
1, & 1, & 1, & 0 \\
\tau / 2, & \tau / 2, & \tau / 2, & 0
\end{array}\right) \\
& d_{3}^{3}=d_{1}^{2} d_{2}^{1}=\omega\left(\begin{array}{ll}
1,1, & 0 \\
0, & 0,0
\end{array}\right)\left(c(q)+\omega\left(\begin{array}{cc}
1, & 0 \\
\tau / 2, & 0
\end{array}\right)\right) \\
& d_{4}^{3}=d_{1}^{1} d_{2}^{2}=\omega\left(\begin{array}{l}
1,0 \\
0,0
\end{array}\right)\left(\frac{1}{2} c(q)^{2}+c(q) \omega\left(\begin{array}{cc}
1, & 0 \\
\tau / 2, & 0
\end{array}\right)+\omega\left(\begin{array}{ccc}
1, & 1, & 0 \\
\tau / 2, \tau / 2, & 0
\end{array}\right)\right) \\
& d_{5}^{3}=d_{1}^{1} d_{1}^{2}=\omega\left(\begin{array}{ll}
1,1,0 \\
0,0,0
\end{array}\right) \omega\left(\begin{array}{ll}
1,0 \\
0,0
\end{array}\right) \\
& d_{7}^{3}=d_{2}^{1} d_{2}^{2}=\left(c(q)+\omega\left(\begin{array}{cc}
1, & 0 \\
\tau / 2, & 0
\end{array}\right)\right)\left(\frac{1}{2} c(q)^{2}+c(q) \omega\left(\begin{array}{cc}
1, & 0 \\
\tau / 2, & 0
\end{array}\right)+\omega\left(\begin{array}{ccc}
1, & 1, & 0 \\
\tau / 2, & \tau / 2, & 0
\end{array}\right)\right) \\
& d_{8}^{3}=d_{1}^{1} d_{3}^{2}=\left(d_{1}^{1}\right)^{2} d_{2}^{1}=\omega\left(\begin{array}{ll}
1, & 0 \\
0, & 0
\end{array}\right)^{2}\left(c(q)+\omega\left(\begin{array}{cc}
1, & 0 \\
\tau / 2, & 0
\end{array}\right)\right) \\
& d_{9}^{3}=d_{2}^{1} d_{3}^{2}=d_{1}^{1}\left(d_{2}^{1}\right)^{2}=\omega\left(\begin{array}{ll}
1, & 0 \\
0, & 0
\end{array}\right)\left(c(q)+\omega\left(\begin{array}{cc}
1, & 0 \\
\tau / 2, & 0
\end{array}\right)\right)^{2} \\
& d_{11}^{3}=\omega\left(\begin{array}{ll}
1, & 0 \\
0, & 0
\end{array}\right)\left(c(q)+\omega\left(\begin{array}{cc}
1, & 0 \\
\tau / 2, & 0
\end{array}\right)\right)^{2}+\frac{1}{3} \omega\left(\begin{array}{lll}
0, & 3, & 0,0 \\
0, & 0,0,0
\end{array}\right) \\
& d_{12}^{3}=d_{2}^{1} d_{5}^{2}=\left(d_{2}^{1}\right)^{3}=\left(c(q)+\omega\left(\begin{array}{cc}
1, & 0 \\
\tau / 2, & 0
\end{array}\right)\right)^{3} .
\end{aligned}
$$

Note that $d_{6}^{2}, d_{6}^{3}$ and $d_{10}^{3}$ are determined by eqs. (4.28) and (4.29), and the derivation of the more involved integral $d_{11}^{3}$ is detailed in appendix I.

\subsubsection{Assembling the orders $\alpha^{\prime \leq 3}$}

Once we apply momentum conservation eq. (4.5) to the integral eq. (4.23), its leading orders in the $\alpha^{\prime}$-expansion simplify to

$$
\begin{aligned}
I_{12 \mid 34}(q)=1 & +2 s_{12}\left(d_{1}^{1}-d_{2}^{1}\right)+s_{12}^{2}\left(2 d_{1}^{2}+2 d_{2}^{2}-4 d_{3}^{2}+d_{4}^{2}+d_{5}^{2}\right)+s_{13} s_{23}\left(2 d_{5}^{2}-4 d_{2}^{2}\right) \\
& +2 s_{12}^{3}\left(d_{1}^{3}-d_{2}^{3}-2 d_{3}^{3}+2 d_{4}^{3}+d_{5}^{3}-d_{7}^{3}-d_{8}^{3}+d_{9}^{3}\right) \\
& -2 s_{12} s_{13} s_{23}\left(d_{12}^{3}-3 d_{2}^{3}+4 d_{4}^{3}-d_{7}^{3}-2 d_{11}^{3}\right)+\mathcal{O}\left(\alpha^{\prime 4}\right) .
\end{aligned}
$$

The $q$-dependence of the relevant teMZVs can be determined by solving the initial-value problem set up in section 3 , yielding for instance

$$
\omega\left(\begin{array}{l}
1,0 \\
0,0
\end{array}\right)=-\frac{i \pi}{2}+2 \sum_{n, m=1}^{\infty} \frac{q^{m n}}{m}, \quad \omega\left(\begin{array}{cc}
1, & 0 \\
\tau / 2, & 0
\end{array}\right)=2 \sum_{n, m=1}^{\infty} \frac{q^{m(n-1 / 2)}}{m},
$$


and further examples can be found in appendix G.4. By comparing the $q$-expansion with the expression eq. (4.14) for $c(q)$, we infer that

$$
c(q)=\omega\left(\begin{array}{l}
1,0 \\
0,0
\end{array}\right)-\omega\left(\begin{array}{cc}
1, & 0 \\
\tau / 2, & 0
\end{array}\right)-\frac{1}{8} \log (q),
$$

which identifies the prefactor $e^{-2 s_{12} c(q)}$ in eq. (4.23) as $q^{s_{12} / 4}$ multiplied by a series in teMZVs $\omega\left(\begin{array}{l}1,0 \\ 0,0\end{array}\right)$ and $\omega\left(\begin{array}{cc}1, & 0 \\ \tau / 2, & 0\end{array}\right)$. Moreover, eq. (4.49) simplifies the order $\alpha^{\prime 1}$ of eq. (4.47) to

$$
\left.I_{12 \mid 34}(q)\right|_{s_{12}}=2\left(d_{1}^{1}-d_{2}^{1}\right)=2\left\{\omega\left(\begin{array}{c}
1,0 \\
0,0
\end{array}\right)-\omega\left(\begin{array}{cc}
1, & 0 \\
\tau / 2, & 0
\end{array}\right)-c(q)\right\}=\frac{1}{4} \log (q),
$$

in agreement with [31]. Also at higher orders of eq. (4.47), we convert any appearance of $c(q)$ into $-\frac{1}{8} \log (q)$ via eq. $(4.49)$ and obtain

$$
\begin{aligned}
& \left.I_{12 \mid 34}(q)\right|_{s_{12}^{2}}=\frac{\left(\log (q)^{2}\right)}{32}+2 \omega\left(\begin{array}{c}
1,1,0 \\
0,0,0
\end{array}\right)-\omega\left(\begin{array}{l}
1,0 \\
0,0
\end{array}\right)^{2}+2 \omega\left(\begin{array}{ccc}
1, & 1, & 0 \\
\tau / 2, & \tau / 2, & 0
\end{array}\right)-\omega\left(\begin{array}{cc}
1, & 0 \\
\tau / 2, & 0
\end{array}\right)^{2} \\
& =\frac{\left(\log (q)^{2}\right)}{32}+\frac{7 \zeta_{2}}{6}+2 \omega(0,0,2) \\
& \left.I_{12 \mid 34}(q)\right|_{s_{13} s_{23}}=2 \omega\left(\begin{array}{cc}
1, & 0 \\
\tau / 2, & 0
\end{array}\right)^{2}-4 \omega\left(\begin{array}{ccc}
1, & 1, & 0 \\
\tau / 2, \tau / 2, & 0
\end{array}\right)=-2 \omega(0,0,2)-\frac{2 \zeta_{2}}{3}
\end{aligned}
$$

via teMZV relations eqs. (G.11) and (G.12) as well as

$$
\begin{aligned}
& \left.I_{12 \mid 34}(q)\right|_{s_{12}^{3}}=\frac{1}{3 !}\left(\frac{\log (q)}{4}\right)^{3}+\frac{1}{4} \log (q)\left(\frac{7 \zeta_{2}}{6}+2 \omega(0,0,2)\right) \\
& +\frac{2}{3} \omega\left(\begin{array}{l}
1,0 \\
0,0
\end{array}\right)^{3}-2 \omega\left(\begin{array}{l}
1,0 \\
0,0
\end{array}\right) \omega\left(\begin{array}{l}
1,1,0 \\
0,0,0
\end{array}\right)+2 \omega\left(\begin{array}{l}
1,1,1,0 \\
0,0,0,0
\end{array}\right) \\
& -\frac{2}{3} \omega\left(\begin{array}{cc}
1, & 0 \\
\tau / 2, & 0
\end{array}\right)^{3}+2 \omega\left(\begin{array}{cc}
1, & 0 \\
\tau / 2, & 0
\end{array}\right) \omega\left(\begin{array}{ccc}
1, & 1, & 0 \\
\tau / 2, \tau / 2, & 0
\end{array}\right)-2 \omega\left(\begin{array}{cccc}
1, & 1, & 1, & 0 \\
\tau / 2, & \tau / 2, & \tau / 2 & 0
\end{array}\right) \\
& =\frac{1}{3 !}\left(\frac{\log (q)}{4}\right)^{3}+\frac{1}{4} \log (q)\left(\frac{7 \zeta_{2}}{6}+2 \omega(0,0,2)\right)-4 \zeta_{2} \omega(0,1,0,0) \\
& \left.I_{12 \mid 34}(q)\right|_{s_{12} s_{23} s_{13}}=\frac{1}{4} \log (q)\left(-2 \omega(0,0,2)-\frac{2 \zeta_{2}}{3}\right)+\frac{4}{3} \omega(0,3,0,0)
\end{aligned}
$$

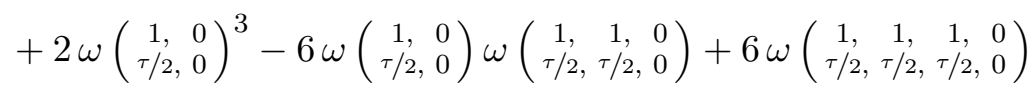

$$
\begin{aligned}
& =\frac{1}{4} \log (q)\left(-2 \omega(0,0,2)-\frac{2 \zeta_{2}}{3}\right)+\frac{5}{3} \omega(0,3,0,0)+4 \zeta_{2} \omega(0,1,0,0)-\frac{1}{2} \zeta_{3}
\end{aligned}
$$

via teMZV relations eqs. (G.13) and (G.14). As will be discussed shortly, the cancellation of teMZVs with nonzero twist manifests the absence of unphysical poles in the string amplitude after integration over $q$.

\subsubsection{Summary of the orders $\alpha^{\prime \leq 3}$}

As is clear by comparing eq. (4.23) with eq. (4.49), any appearance of $\log (q)$ can be traced back to the expansion of $q^{s_{12} / 4}$. Hence, the above orders $\alpha^{\prime \leq 3}$ can be summarized as

$$
\begin{aligned}
I_{12 \mid 34}(q)= & q^{s_{12} / 4}\left\{1+s_{12}^{2}\left(\frac{7 \zeta_{2}}{6}+2 \omega(0,0,2)\right)-2 s_{13} s_{23}\left(\frac{\zeta_{2}}{3}+\omega(0,0,2)\right)\right. \\
& \left.-4 \zeta_{2} \omega(0,1,0,0) s_{12}^{3}+s_{12} s_{13} s_{23}\left(\frac{5}{3} \omega(0,3,0,0)+4 \zeta_{2} \omega(0,1,0,0)-\frac{1}{2} \zeta_{3}\right)+\mathcal{O}\left(\alpha^{\prime 4}\right)\right\},
\end{aligned}
$$


and the integral $I_{123 \mid 4}(q)$ in eq. (4.6) admits a similar low-energy expansion in terms of eMZVs only, see eq. (H.26). While the $q$-expansions of the above eMZVs are listed in appendix G.4, their constant terms yield

$$
I_{12 \mid 34}(q)=q^{s_{12} / 4}\left\{1+\frac{1}{2} \zeta_{2} s_{12}^{2}-\frac{1}{2} \zeta_{3} s_{12}^{3}+\mathcal{O}\left(q, \alpha^{\prime 4}\right)\right\},
$$

in agreement with the all-order $\alpha^{\prime}$ expression given in 31

$$
I_{12 \mid 34}(q)=\frac{2^{2 s_{12}} q^{s_{12} / 4}}{\pi}\left\{\left(\frac{\Gamma\left(\frac{1}{2}+\frac{s_{12}}{2}\right)}{\Gamma\left(1+\frac{s_{12}}{2}\right)}\right)^{2}+\mathcal{O}(q)\right\} .
$$

With the above strategy and the techniques exemplified in appendix I, there is no limitation in obtaining higher orders of the $\alpha^{\prime}$-expansion eq. (4.55). Similarly, non-planar open-string amplitudes with five and more external legs can be expanded along the same lines ${ }^{16}$ because their integrands depend on the punctures and $\tau$ through products of $f^{(n)}$ and Eisenstein series $[58,59,30]$.

\subsubsection{Higher orders in $\alpha^{\prime}$ and eMZVs}

The exclusive appearance of eMZVs at the leading orders of the $\alpha^{\prime}$-expansion eq. (4.55) illustrates a general property of the non-planar integral $I_{12 \mid 34}(q)$ : apart from the prefactor $q^{s_{12} / 4}$, its $q$ expansion comprises integer powers only. This property is shared by eMZVs but not by typical teMZVs involving twists $\tau / 2$.

The absence of half-odd integer powers $q^{n+1 / 2}$ with $n \in \mathbb{N}_{0}$ can be explained from physical constraints on the pole structure of the open-string amplitude: Performing the $q$-integration in the amplitude prescription eq. (4.1) over expansions of the schematic form

$$
I_{12 \mid 34}(q)=q^{s_{12} / 4} \sum_{n=0}^{\infty}\left(a_{n} q^{n}+c_{n} q^{n+1 / 2}\right)
$$

yields kinematic poles at $s_{12}=-4 n$ in case of integer powers of $q$ and at $s_{12}=-4 n-2$ in case of half-odd integer powers, respectively, with $n \in \mathbb{N}_{0}$ :

$$
\int_{0}^{1} \frac{\mathrm{d} q}{q} I_{12 \mid 34}(q)=\sum_{n=0}^{\infty}\left\{\frac{4 a_{n}}{s_{12}+4 n}+\frac{4 c_{n}}{s_{12}+4 n+2}\right\} .
$$

The expansion coefficients $a_{n}$ and $c_{n}$ are understood to be formal power series in the Mandelstam variables $s_{i j}$ accompanied by $\mathbb{Q}\left[(2 \pi i)^{-1}\right]$-linear combinations of MZVs.

The singular values of Mandelstam variables $s_{12}=\alpha^{\prime}\left(k_{1}+k_{2}\right)^{2}$ in scattering amplitudes with external momenta $k_{i}$ correspond to internal masses $-\alpha^{\prime} m^{2}$. As a general property of non-planar one-loop open-string string amplitudes, the kinematic poles arising from the integration over $q$ reveal the appearance of closed-string modes among the internal states 44 . In particular, the poles in eq. (4.59) with residues proportional to $a_{n}$ and $c_{n}$ signal the exchange of closed-string states with masses $m^{2}=\frac{4 n}{\alpha^{\prime}}$ and $m^{2}=\frac{4 n+2}{\alpha^{\prime}}$, respectively, with $n \in \mathbb{N}_{0}$. However, the closedsuperstring spectrum only comprises masses $m^{2}=\frac{4 n}{\alpha^{\prime}}$, whereas states with $m^{2}=\frac{4 n+2}{\alpha^{\prime}}$ cannot be found in GSO projected string theories $43,44,46,47$. Hence, the pole structure of $\frac{4 c_{n}}{s_{12}+4 n+2}$

\footnotetext{
${ }^{16}$ See section 5.1 of 30 for the analogous expansion of the planar five-point one-loop amplitude in terms of eMZVs.
} 
due to half-odd integer powers of $q$ would signal the propagation of unphysical states and violate unitarity if some of the $c_{n}$ were nonzero.

However, it is not immediately clear if an integer-power $q$-expansion eq. $(4.58)$ with $c_{n}=0$ is necessarily expressible in terms of eMZVs. Given that $q^{-s_{12} / 4} I_{12 \mid 34}(q)$ was argued to comprise teMZVs with twists $\in\{0, \tau / 2\}$, this leads to the following, purely mathematical question: If a linear combination of teMZVs with twists $\in\{0, \tau / 2\}$ is such that its $q$-expansion has integer exponents only, can it be written as a linear combination of eMZVs (i.e. teMZVs with vanishing twists) only?

We expect that an answer to this question will necessitate a closer study of the decomposition of teMZVs into iterated $\tau$-integrals of the functions $h^{(n)}(b)$ in eq. (3.4), with $b \in\{0, \tau / 2\}$. Given that $h^{(n)}(b)$ are modular forms for congruence subgroups of $\mathrm{SL}_{2}(\mathbb{Z})$, this decomposition of teMZVs generalizes the decomposition of eMZVs into linear combinations of iterated Eisenstein integrals $[10,36]$. In particular, a natural first step would be to prove linear independence of iterated $\tau$-integrals comprised of the integrands $h^{(n)}(b)$ with $b \in\{0, \tau / 2\}$, which would generalize results of $[12,60$, and can presumably be proved along similar lines.

In any case, based on the arguments presented in this subsection, we conjecture that all orders in the $\alpha^{\prime}$-expansion eq. (4.55) are furnished by eMZVs. The same is expected to hold for all non-planar $n$-point amplitudes at one loop prior to integration over $q$. It is conceivable that this can be derived from the one-loop monodromy relations [53,31], and it would be interesting to work out a rigorous proof.

\section{Conclusions}

In this article, teMZVs have been introduced as iterated integrals on an elliptic curve with multiple punctures on a lattice $\mathbb{Q}+\mathbb{Q} \tau$. Our main result is the identification of an initial-value problem satisfied by teMZVs, which expresses them in terms of linear combinations of iterated $\tau$-integrals of the weighting functions $f^{(n)}$ with coefficients given by cyclotomic MZVs.

As an application of teMZVs in physics we have studied one-loop scattering amplitudes of open-string states. In the non-planar sector of the four-point amplitude, the low-energy expansion can be computed by integrals over the two boundaries of a cylinder. A systematic procedure is established, which allows to evaluate these integrals in terms of teMZVs. There is no conceptual bottleneck in extending the procedure to one-loop amplitudes with an arbitrary number of external states. Having calculated the non-planar amplitude up to the third subleading low-energy order, we find that the results can ultimately be simplified to eMZVs.

The results of this article trigger a variety of questions: From a mathematical perspective, the differential equation of teMZVs could serve as a starting point to classify their relations and to understand the underlying algebraic principles. In the untwisted case, a crucial rôle was played by a certain derivation algebra. We expect that a suitable twisted analogue of the derivation algebra $[9]$ will likewise control the algebraic structure of teMZVs.

In a physics context, the methods of this article allow to compute higher orders in the lowenergy expansion of non-planar one-loop open-string amplitudes and to investigate its structure. More interestingly, higher-loop open-string amplitudes should require an extension of elliptic iterated integrals to Riemann surfaces of higher genus and a suitable generalization of teMZVs to accommodate multiple boundaries. 


\section{Acknowledgments}

We would like to thank Claire Glanois, Martin Gonzalez, David Meidinger and Federico Zerbini for helpful discussions, and the Kolleg Mathematik und Physik Berlin for support in various ways. JB and OS would like to thank Universität Hamburg for hospitality. The work of JB and GR is supported in part by the SFB 647 "Raum-Zeit-Materie. Analytische und Geometrische Strukturen". GR is furthermore supported by the International Max Planck Research School for Mathematical and Physical Aspects of Gravitation, Cosmology and Quantum Field Theory. This paper was written while NM was a Ph.D. student at Universität Hamburg under the supervision of Ulf Kühn. The research of OS was supported in part by Perimeter Institute for Theoretical Physics. Research at Perimeter Institute is supported by the Government of Canada through the Department of Innovation, Science and Economic Development and by the Province of Ontario through the Ministry of Research, Innovation and Science.

\section{Appendix}

\section{A Jacobi theta functions}

For later use we note as well the expansion of Jacobi theta functions (and derivatives thereof) as an expansion in the parameter $q=e^{2 \pi i \tau} \mid 57$

$$
\begin{aligned}
& \theta_{1}(z, \tau)=2 q^{1 / 8} \sin (\pi z) \prod_{n=1}^{\infty}\left(1-q^{n}\right)\left(1-2 q^{n} \cos (2 \pi z)+q^{2 n}\right) \\
& \theta_{1}^{\prime}(0, \tau)=2 \pi q^{1 / 8} \prod_{n=1}^{\infty}\left(1-q^{n}\right)^{3} \\
& \theta_{4}(z, \tau)=\prod_{n=1}^{\infty}\left(1-q^{n}\right)\left(1-2 q^{n-1 / 2} \cos (2 \pi z)+q^{2 n-1}\right),
\end{aligned}
$$

which all turn out to be positive given $z \in[0,1]$ and $q \in[0,1]$. Furthermore, a periodicity property useful for calculating eq. (4.18) as well as a relation between $\theta_{1}$ and $\theta_{4}$ reads

$$
\begin{aligned}
\theta_{1}(z+m+n \tau, \tau) & =(-1)^{n+m} q^{-n^{2} / 2} e^{-2 \pi i n z} \theta_{1}(z, \tau) \\
\theta_{4}(z, \tau) & =-i e^{i \pi z} q^{1 / 8} \theta_{1}(z+\tau / 2, \tau) .
\end{aligned}
$$

\section{B Weighting functions}

The weighting functions $f^{(n)}(z, \tau)$ arise as expansion coefficients of the doubly-periodic completion $\Omega(z, \alpha, \tau)$ of the Eisenstein-Kronecker series $F(z, \alpha, \tau)$,

$$
\Omega(z, \alpha, \tau)=\exp \left(2 \pi i \alpha \frac{\operatorname{Im}(z)}{\operatorname{Im}(\tau)}\right) F(z, \alpha, \tau)=\sum_{n=0}^{\infty} f^{(n)}(z, \tau) \alpha^{n-1},
$$

which, in turn is given by 61,6

$$
F(z, \alpha, \tau)=\frac{\theta_{1}^{\prime}(0, \tau) \theta_{1}(z+\alpha, \tau)}{\theta_{1}(z, \tau) \theta_{1}(\alpha, \tau)} .
$$


The odd Jacobi theta function $\theta_{1}$ is defined in appendix $\mathrm{A}$, and the derivative with respect to the first argument is denoted by a tick. For real $z$, the expressions in eqs. (B.1) and (B.2) agree, and the lowest-order examples of $f^{(n)}$ are spelt out in eq. (2.2). In fact, $f^{(1)}$ is the only weighting function with a simple pole on the lattice $\mathbb{Z}+\mathbb{Z} \tau$, while all $f^{(n)}$ with $n \neq 1$ are non-singular on the entire elliptic curve.

Both the Eisenstein-Kronecker series $F(z, \alpha, \tau)$ and its doubly periodic completion $\Omega(z, \alpha, \tau)$ satisfy the Fay identity

$$
\begin{aligned}
\Omega\left(z_{1}, \alpha_{1}, \tau\right) \Omega\left(z_{2}, \alpha_{2}, \tau\right)= & \Omega\left(z_{1}, \alpha_{1}+\alpha_{2}, \tau\right) \Omega\left(z_{2}-z_{1}, \alpha_{2}, \tau\right) \\
& +\Omega\left(z_{2}, \alpha_{1}+\alpha_{2}, \tau\right) \Omega\left(z_{1}-z_{2}, \alpha_{1}, \tau\right) .
\end{aligned}
$$

Furthermore, the Eisenstein-Kronecker series satisfies the mixed heat equation

$$
2 \pi i \partial_{\tau} F(z, \alpha, \tau)=\partial_{\alpha} \partial_{z} F(z, \alpha, \tau)
$$

Both the Fay identity eq. (B.3) and the mixed heat equation (B.4) are relevant in the calculations of section 3. Starting from the quasi-periodicity of the Eisenstein-Kronecker series,

$$
F(z+1, \alpha, \tau)=F(z, \alpha, \tau), \quad F(z+\tau, \alpha, \tau)=\exp (-2 \pi i \alpha) F(z, \alpha, \tau)
$$

and its reflection property

$$
F(-z,-\alpha, \tau)=-F(z, \alpha, \tau)
$$

it is straightforward to derive properties eq. $(2.3)$ of the weighting functions $f^{(n)}$.

\section{A teMZV with proper rational twist $b=1 / 2$}

We illustrate the definition of teMZVs in the case of proper rational twists $b \in \Lambda_{N}^{\times}=\left\{\frac{1}{N}, \ldots, \frac{N-1}{N}\right\}$, via an explicit computation of $\omega\left(\begin{array}{cc}0 & 1 \\ 0, & 1 / 2\end{array}\right)$. Our starting point is the definition eq. (2.7) of teMZVs with twists $b_{i} \in\{0,1 / 2\}$ through the integral

$$
\omega\left(\begin{array}{c}
n_{1}, n_{2}, \ldots, n_{\ell} \\
b_{1}, b_{2}, \ldots, b_{\ell}
\end{array}\right)=\lim _{\varepsilon \rightarrow 0} \int_{\alpha_{1} \delta_{\varepsilon} \alpha_{2}} f^{\left(n_{1}\right)}\left(z-b_{1}\right) \mathrm{d} z_{1} f^{\left(n_{2}\right)}\left(z-b_{2}\right) \mathrm{d} z_{2} \ldots f^{\left(n_{\ell}\right)}\left(z-b_{\ell}\right) \mathrm{d} z_{\ell} .
$$

We choose the parametrization of the individual path segments depicted in figure 3 as

$$
\begin{aligned}
\alpha_{1}(t) & =\left(\frac{1}{2}-\varepsilon\right) t \\
\delta_{\varepsilon}(t) & =\frac{1}{2}-\varepsilon \exp (-i \pi t) \\
\alpha_{2}(t) & =\frac{1}{2}+\varepsilon+\left(\frac{1}{2}-\varepsilon\right) t
\end{aligned}
$$

with $t \in(0,1)$ in each case. Then we may compute the iterated integral using the composition of paths formula, for the smooth one-forms $\omega_{i}=f^{\left(n_{i}\right)}\left(z-b_{i}\right) \mathrm{d} z$ (cf. 62, Proposition 2.9)

$$
\int_{\alpha \beta} \omega_{1} \omega_{2} \ldots \omega_{\ell}=\sum_{k=0}^{\ell} \int_{\alpha} \omega_{1} \omega_{2} \ldots \omega_{k} \int_{\beta} \omega_{k+1} \ldots \omega_{\ell}
$$

where the paths $\alpha, \beta$ are such that $\alpha(1)=\beta(0)$ and the empty integral is defined to be one. 
As the forms $\omega_{i}=f^{\left(n_{i}\right)}\left(z-b_{i}\right) \mathrm{d} z$ admit an expansion in $q$ we may treat the $q^{0}$ term separately from the rest, assuming the $q$-expansion can be exchanged with the integration. Then, as the coefficients of the $j^{\text {th }}$ power $q^{j}$ for $j \neq 0$ are well defined on the real line, we may exchange the limit $\varepsilon \rightarrow 0$ with the integration and compute this part of the integral over the much more mundane path $\gamma(t)=t$. Specifically, the $q$ dependent part is given by

$$
I_{q}=-2 i(2 \pi i) \int_{0<t_{1}<t_{2}<1} \mathrm{~d} t_{1} \mathrm{~d} t_{2} \sum_{n, m=1}^{\infty} q^{m n} \sin \left(2 \pi m\left(t_{2}-\frac{1}{2}\right)\right)=-2 \sum_{n, m=1}^{\infty} \frac{(-1)^{m} q^{m n}}{m} .
$$

We note that this can be reproduced from the differential equation $(\overline{3.5})$ for real twists

$$
\begin{aligned}
& \omega\left(\begin{array}{cc}
0, & 1 \\
0,1 / 2
\end{array}\right)=\omega_{0}\left(\begin{array}{cc}
0, & 1 \\
0,1 / 2
\end{array}\right)+\int_{0}^{q} \frac{\mathrm{d} \log \left(q_{1}\right)}{-4 \pi^{2}}\left[\omega\left(\begin{array}{c}
2 \\
1 / 2
\end{array}\right)-f^{(2)}\left(\frac{1}{2}, q_{1}\right)\right] \\
& =\omega_{0}\left(\begin{array}{cc}
0, & 1 \\
0,1 / 2
\end{array}\right)+\int_{0}^{q} \frac{\mathrm{d} \log \left(q_{1}\right)}{-4 \pi^{2}}\left[8 \pi^{2} \sum_{m, n=1}^{\infty}(-1)^{m} n q_{1}^{m n}\right],
\end{aligned}
$$

where the integration constant $\omega_{0}\left(\begin{array}{ll}0, & 1 \\ 0, & 1 / 2\end{array}\right)$ remains to be determined.

Application of eq. (C.3) for the constant term $f_{0}^{(n)}(z-b)$ of the $q$ expansion of $f^{(n)}(z-b)$ yields, bearing in mind that $f^{(0)}(z)=1$,

$$
\begin{aligned}
I_{0}= & \int_{\alpha_{1} \delta_{\varepsilon} \alpha_{2}} \mathrm{~d} z_{1} f_{0}^{(1)}\left(z_{2}-\frac{1}{2}\right) \mathrm{d} z_{2} \\
= & \int_{\alpha_{1}} \mathrm{~d} z_{1} f_{0}^{(1)}\left(z_{2}-\frac{1}{2}\right) \mathrm{d} z_{2}+\int_{\delta_{\varepsilon}} \mathrm{d} z_{1} f_{0}^{(1)}\left(z_{2}-\frac{1}{2}\right) \mathrm{d} z_{2}+\int_{\alpha_{2}} \mathrm{~d} z_{1} f_{0}^{(1)}\left(z_{2}-\frac{1}{2}\right) \mathrm{d} z_{2} \\
& +\int_{\alpha_{1}} \mathrm{~d} w \int_{\delta_{\varepsilon}} f_{0}^{(1)}\left(z-\frac{1}{2}\right) \mathrm{d} z+\int_{\alpha_{1}} \mathrm{~d} w \int_{\alpha_{2}} f_{0}^{(1)}\left(z-\frac{1}{2}\right) \mathrm{d} z+\int_{\delta_{\varepsilon}} \mathrm{d} w \int_{\alpha_{2}} f_{0}^{(1)}\left(z-\frac{1}{2}\right) \mathrm{d} z .
\end{aligned}
$$

The individual integrals are given by

$$
\begin{aligned}
\int_{\alpha_{1}} \mathrm{~d} z_{1} f_{0}^{(1)}\left(z_{2}-\frac{1}{2}\right) \mathrm{d} z_{2} & =\int_{0<t_{1}<t_{2}<1} \mathrm{~d} t_{1} \mathrm{~d} t_{2}\left(\frac{1}{2}-\varepsilon\right)^{2} \pi \cot \left(\pi\left[\left(\frac{1}{2}-\varepsilon\right) t_{2}-\frac{1}{2}\right]\right) \\
& =\frac{\log (2)}{2}+\frac{\log (\pi \varepsilon)}{2}+\mathcal{O}(\varepsilon) \\
\int_{\delta_{\varepsilon}} \mathrm{d} z_{1} f_{0}^{(1)}\left(z_{2}-\frac{1}{2}\right) \mathrm{d} z_{2}= & \int_{0<t_{1}<t_{2}<1} \mathrm{~d} t_{1} \mathrm{~d} t_{2}(i \pi)^{2} \varepsilon^{2} e^{-i \pi\left(t_{1}+t_{2}\right)} \pi \cot \left(-\pi \varepsilon e^{-i \pi t_{2}}\right) \\
= & \mathcal{O}(\varepsilon) \\
\int_{\alpha_{2}} \mathrm{~d} z_{1} f_{0}^{(1)}\left(z_{2}-\frac{1}{2}\right) \mathrm{d} z_{2}= & \int_{0<t_{1}<t_{2}<1} \mathrm{~d} t_{1} \mathrm{~d} t_{2}\left(\frac{1}{2}-\varepsilon\right)^{2} \pi \cot \left(\pi\left[\left(\frac{1}{2}-\varepsilon\right) t_{2}+\varepsilon\right]\right) \\
= & \frac{\log (2)}{2}+\varepsilon \log (\pi \varepsilon)+\mathcal{O}(\varepsilon) \\
\int_{\alpha_{1}} \mathrm{~d} w \int_{\delta_{\varepsilon}} f_{0}^{(1)}\left(z-\frac{1}{2}\right) \mathrm{d} z= & i \pi\left(\varepsilon-\frac{1}{2}\right) \\
\int_{\alpha_{1}} \mathrm{~d} w \int_{\alpha_{2}} f_{0}^{(1)}\left(z-\frac{1}{2}\right) \mathrm{d} z= & \left(\varepsilon-\frac{1}{2}\right) \log (\sin (\pi \varepsilon))
\end{aligned}
$$




$$
\int_{\delta_{\varepsilon}} \mathrm{d} w \int_{\alpha_{2}} f_{0}^{(1)}\left(z-\frac{1}{2}\right) \mathrm{d} z=-2 \varepsilon \log (\sin (\pi \varepsilon)) .
$$

Note that due to $\lim _{\varepsilon \rightarrow 0} \varepsilon \log (\sin (\pi \varepsilon))=0$ the only singular contributions stem from the integrals in eqs. (C.7) and (C.11), which cancel in their sum. Then, we arrive at

$$
\lim _{\varepsilon \rightarrow 0} I_{0}=-\frac{i \pi}{2}+\log (2)
$$

as predicted by the constant-term procedure eq. (3.27), see eq. (G.7). Finally, upon combination with the $q$-series in eq. (C.4), the desired teMZV is given by

$$
\omega\left(\begin{array}{cc}
0, & 1 \\
0,1 / 2
\end{array}\right)=\lim _{\varepsilon \rightarrow 0}\left(I_{0}+I_{q}\right)=-\frac{i \pi}{2}+\log (2)-2 \sum_{n, m=1}^{\infty} \frac{(-1)^{m} q^{m n}}{m} .
$$

\section{MZVs and cyclotomic MZVs}

Cyclotomic MZVs (also called "multiple polylogarithms at roots of unity") are generalizations of MZVs. While MZVs are represented by nested sums of the form

$$
\zeta_{n_{1}, n_{2}, \ldots, n_{r}}=\zeta\left(n_{1}, n_{2}, \ldots, n_{r}\right)=\sum_{0<k_{1}<k_{2}<\ldots<k_{r}}^{\infty} \frac{1}{k_{1}^{n_{1}} k_{2}^{n_{2}} \ldots k_{r}^{n_{r}}}, \quad n_{1}, \ldots, n_{r-1} \geq 1, n_{r} \geq 2
$$

cyclotomic MZVs are represented by nested sums with additional "coloring" given by $N^{\text {th }}$ roots of unity $\sigma_{1}, \ldots, \sigma_{r} \in \mu_{N}$ :

$$
\zeta\left(\begin{array}{c}
n_{1}, n_{2}, \ldots, n_{r} \\
\sigma_{1}, \sigma_{2}, \ldots, \sigma_{r}
\end{array}\right)=\sum_{0<k_{1}<k_{2}<\ldots<k_{r}} \frac{\sigma_{1}^{k_{1}} \sigma_{2}^{k_{2}} \ldots \sigma_{r}^{k_{r}}}{k_{1}^{n_{1}} k_{2}^{n_{2}} \ldots k_{r}^{n_{r}}}, \quad n_{1}, \ldots, n_{r} \geq 1, n_{r} \geq 2 \text { if } \sigma_{r}=1 .
$$

Likewise, the integral representation of MZVs

$$
\zeta\left(n_{1}, \ldots, n_{r}\right)=\int_{0 \leq t_{i} \leq t_{i+1} \leq 1} \frac{\mathrm{d} t_{1}}{1-t_{1}} \frac{\mathrm{d} t_{2}}{t_{2}} \ldots \frac{\mathrm{d} t_{n_{1}}}{t_{n_{1}}} \frac{\mathrm{d} t_{n_{1}+1}}{1-t_{n_{1}+1}} \ldots \frac{\mathrm{d} t_{w}}{t_{w}}, \quad w=n_{1}+\ldots+n_{r},
$$

generalizes to an integral representation for cyclotomic MZVs

$$
\zeta\left(\begin{array}{c}
n_{1}, n_{2}, \ldots, n_{r} \\
\sigma_{1}, \sigma_{2}, \ldots, \sigma_{r}
\end{array}\right)=\int_{0 \leq t_{i} \leq t_{i+1} \leq 1} \frac{\mathrm{d} t_{1}}{\eta_{1}-t_{1}} \frac{\mathrm{d} t_{2}}{t_{2}} \ldots \frac{\mathrm{d} t_{n_{1}}}{t_{n_{1}}} \frac{\mathrm{d} t_{n_{1}+1}}{\eta_{2}-t_{n_{1}+1}} \ldots \frac{\mathrm{d} t_{w}}{t_{w}}
$$

where $\eta_{i}=\left(\sigma_{i} \sigma_{i+1} \cdots \sigma_{r}\right)^{-1}$. The positive integer $N$, implicit in the definition of cyclotomic MZVs, is sometimes considered an additional datum, and one speaks of $N$-cyclotomic MZVs to emphasize the choice of $N$.

Cyclotomic MZVs have first been considered by Goncharov [3. Suitable references for cyclotomic MZVs include $13-15$. For a detailed study of $N$-cyclotomic MZVs where $N=2,3,4,6,8$, see [63. More recently, the case $N=6$ has generated further interest 64-66]. 


\section{E Details on the differential equation of teMZVs}

In this appendix, we give a detailed derivation of the differential equation (3.5) in the case $b_{1}, b_{\ell} \neq 0$. The case $b_{1}=0$ or $b_{\ell}=0$ is technically more complicated, since the iterated integrals involved need to be regularized according to ref. 8. However, using Proposition 3.1 of [8], the arguments of this section go through for $b_{1}=0$ or $b_{\ell}=0$ as well.

Using the mixed heat equation $(3.2)$ for $\Omega(z-b, \alpha)$, we may rewrite the $\tau$-derivative of the generating function eq. (3.1) of length- $\ell$ teMZVs as

$$
\begin{aligned}
& 2 \pi i \frac{\partial}{\partial \tau} \mathrm{T}\left[\begin{array}{c}
\alpha_{1}, \alpha_{2}, \ldots, \alpha_{\ell} \\
b_{1}, b_{2}, \ldots, b_{\ell}
\end{array}\right]=\int_{0 \leq z_{p} \leq z_{p+1} \leq 1} \mathrm{~d} z_{1} \mathrm{~d} z_{2} \ldots \mathrm{d} z_{\ell} \sum_{i=1}^{\ell} \partial_{\alpha_{i}} \partial_{z_{i}} \Omega\left(z_{i}-b_{i}, \alpha_{i}\right) \prod_{j \neq i}^{\ell} \Omega\left(z_{j}-b_{j}, \alpha_{j}\right) \\
& =\int_{0 \leq z_{p} \leq z_{p+1} \leq 1} \mathrm{~d} z_{2} \ldots \mathrm{d} z_{\ell} \partial_{\alpha_{1}} \Omega\left(z_{2}-b_{1}, \alpha_{1}\right) \prod_{j=2}^{\ell} \Omega\left(z_{j}-b_{j}, \alpha_{j}\right)-\partial_{\alpha_{1}} \Omega\left(-b_{1}, \alpha_{1}\right) \mathrm{T}\left[\begin{array}{l}
\alpha_{2}, \ldots, \alpha_{\ell} \\
b_{2}, \ldots, b_{\ell}
\end{array}\right] \\
& +\partial_{\alpha_{\ell}} \Omega\left(-b_{\ell}, \alpha_{\ell}\right) \mathrm{T}\left[\begin{array}{c}
\alpha_{1}, \ldots, \alpha_{\ell-1} \\
b_{1}, \ldots, b_{\ell-1}
\end{array}\right]-\int_{0 \leq z_{p} \leq z_{p+1} \leq 1} \mathrm{~d} z_{1} \ldots \mathrm{d} z_{\ell-1} \partial_{\alpha_{\ell}} \Omega\left(z_{\ell-1}-b_{\ell}, \alpha_{\ell}\right) \prod_{j=1}^{\ell-1} \Omega\left(z_{j}-b_{j}, \alpha_{j}\right) \\
& +\left.\sum_{i=2}^{\ell-1} \int_{0 \leq z_{p} \leq z_{p+1} \leq 1} \mathrm{~d} z_{1} \ldots \mathrm{d} z_{i-1} \mathrm{~d} z_{i+1} \ldots \mathrm{d} z_{r} \partial_{\alpha_{i}} \Omega\left(z_{i}-b_{i}, \alpha_{i}\right)\right|_{z_{i}=z_{i-1}} ^{z_{i}=z_{i+1}} \prod_{j \neq i}^{\ell} \Omega\left(z_{j}-b_{j}, \alpha_{j}\right) \\
& =\partial_{\alpha_{\ell}} \Omega\left(-b_{\ell}, \alpha_{\ell}\right) \mathrm{T}\left[\begin{array}{c}
\alpha_{1}, \ldots, \alpha_{\ell-1} \\
b_{1}, \ldots,
\end{array} b_{\ell-1}\right]-\partial_{\alpha_{1}} \Omega\left(-b_{1}, \alpha_{1}\right) \mathrm{T}\left[\begin{array}{ccc}
\alpha_{2} & \ldots & \alpha_{\ell} \\
b_{2} & \ldots & b_{\ell}
\end{array}\right] \\
& +\int_{0 \leq z_{p} \leq z_{p+1} \leq 1} \mathrm{~d} z_{2} \mathrm{~d} z_{3} \ldots \mathrm{d} z_{\ell} \sum_{i=2}^{\ell} \prod_{j \neq i, 1}^{\ell} \Omega\left(z_{j}-b_{j}, \alpha_{j}\right)\left(\partial_{\alpha_{i-1}}-\partial_{\alpha_{i}}\right) \Omega\left(z_{i}-b_{i-1}, \alpha_{i-1}\right) \Omega\left(z_{i}-b_{i}, \alpha_{i}\right) \\
& =\partial_{\alpha_{\ell}} \Omega\left(-b_{\ell}, \alpha_{\ell}\right) \mathrm{T}\left[\begin{array}{l}
\alpha_{1}, \ldots, \alpha_{\ell-1} \\
b_{1}, \ldots, b_{\ell-1}
\end{array}\right]-\partial_{\alpha_{1}} \Omega\left(-b_{1}, \alpha_{1}\right) \mathrm{T}\left[\begin{array}{c}
\alpha_{2}, \ldots, \\
b_{2}, \ldots, b_{\ell}
\end{array}\right]
\end{aligned}
$$

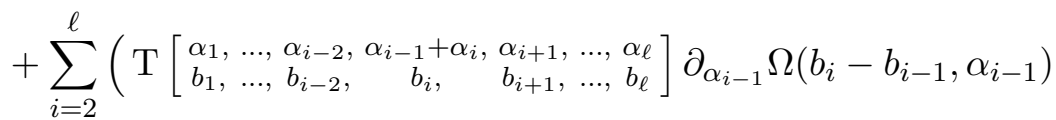

$$
\begin{aligned}
& \left.-\mathrm{T}\left[\begin{array}{ccc}
\alpha_{1}, \ldots, \alpha_{i-2}, & \alpha_{i-1}+\alpha_{i}, & \alpha_{i+1}, \ldots, \\
b_{1}, \ldots, & \alpha_{\ell-2} \\
b_{i-2} & b_{i-1}, & b_{i+1}, \ldots, \\
b_{\ell}
\end{array}\right] \partial_{\alpha_{i}} \Omega\left(b_{i-1}-b_{i}, \alpha_{i}\right)\right),
\end{aligned}
$$

where we mapped the integrals over $\partial_{z_{i}}(\ldots)$ to boundary terms in the second equality and used the Fay identity (B.3) in the last equality to simplify

$$
\begin{aligned}
& \left(\partial_{\alpha_{i-1}}-\partial_{\alpha_{i}}\right) \Omega\left(z_{i}-b_{i-1}, \alpha_{i-1}\right) \Omega\left(z_{i}-b_{i}, \alpha_{i}\right) \\
& =\left(\partial_{\alpha_{i-1}}-\partial_{\alpha_{i}}\right)\left[\Omega\left(z_{i}-b_{i}, \alpha_{i-1}+\alpha_{i}\right) \Omega\left(b_{i}-b_{i-1}, \alpha_{i-1}\right)+\Omega\left(z_{i}-b_{i-1}, \alpha_{i-1}+\alpha_{i}\right) \partial_{\alpha_{i}} \Omega\left(b_{i-1}-b_{i}, \alpha_{i}\right)\right] \\
& =\Omega\left(z_{i}-b_{i}, \alpha_{i-1}+\alpha_{i}\right) \partial_{\alpha_{i-1}} \Omega\left(b_{i}-b_{i-1}, \alpha_{i-1}\right)-\Omega\left(z_{i}-b_{i-1}, \alpha_{i-1}+\alpha_{i}\right) \partial_{\alpha_{i}} \Omega\left(b_{i-1}-b_{i}, \alpha_{i}\right)
\end{aligned}
$$

From the above differential equation for the generating function one may deduce a differential 
equation for teMZVs,

$$
\begin{aligned}
& 2 \pi i \partial_{\tau} \mathrm{T}\left[\begin{array}{l}
\alpha_{1}, \alpha_{2}, \ldots, \alpha_{\ell} \\
b_{1}, b_{2}, \ldots, b_{\ell}
\end{array}\right]=2 \pi i \sum_{n_{1}, n_{2}, \ldots, n_{\ell}=0}^{\infty} \alpha_{1}^{n_{1}-1} \ldots \alpha_{\ell}^{n_{\ell}-1} \partial_{\tau} \omega\left(\begin{array}{l}
n_{1}, \ldots, n_{\ell} \\
b_{1}, \ldots, b_{\ell}
\end{array}\right) \\
& =\partial_{\alpha_{\ell}} \Omega\left(-b_{\ell}, \alpha_{\ell}\right) \mathrm{T}\left[\begin{array}{c}
\alpha_{1}, \ldots, \alpha_{\ell-1} \\
b_{1}, \ldots, b_{\ell-1}
\end{array}\right]-\partial_{\alpha_{1}} \Omega\left(-b_{1}, \alpha_{1}\right) \mathrm{T}\left[\begin{array}{l}
\alpha_{2}, \ldots, \alpha_{\ell} \\
b_{2}, \ldots, b_{\ell}
\end{array}\right]
\end{aligned}
$$

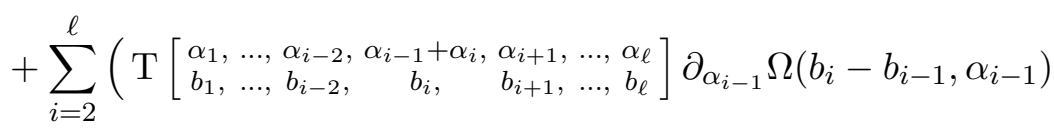



Eventually we want to equate coefficients to extract the $\tau$-derivative of a particular teMZV. For this purpose let us consider the terms in eq. (E.3) separately. Recalling the definition eq. (3.4) of $h^{(n)}$ we may rewrite the first term on the right hand side of eq. (E.3) as

$$
\begin{array}{r}
\partial_{\alpha_{\ell}} \Omega\left(-b_{\ell}, \alpha_{\ell}\right) \mathrm{T}\left[\begin{array}{l}
\alpha_{1}, \ldots, \alpha_{\ell-1} \\
b_{1}, \ldots, b_{\ell-1}
\end{array}\right]= \\
\sum_{n_{\ell}=0}^{\infty} h^{\left(n_{\ell}\right)}\left(-b_{\ell}\right) \alpha_{\ell}^{n_{\ell}-2} \sum_{n_{1}, n_{2}, \ldots, n_{\ell-1}=0}^{\infty} \alpha_{1}^{n_{1}-1} \ldots \alpha_{\ell-1}^{n_{\ell-1}-1} \omega\left(\begin{array}{l}
n_{1}, \ldots, n_{\ell-1} \\
b_{1}, \ldots, b_{\ell-1}
\end{array}\right) \\
=\sum_{n_{1}, n_{2}, \ldots, n_{\ell}=0}^{\infty} \alpha_{1}^{n_{1}-1} \ldots \alpha_{\ell-1}^{n_{\ell-1}-1} \alpha_{\ell}^{n_{\ell}-1} h^{\left(n_{\ell}+1\right)}\left(-b_{\ell}\right) \omega\left(\begin{array}{l}
n_{1}, \ldots, n_{\ell-1} \\
b_{1}, \ldots, b_{\ell-1}
\end{array}\right) \\
+h^{(0)}\left(-b_{\ell}\right) \alpha_{\ell}^{-2} \sum_{n_{1}, n_{2}, \ldots, n_{\ell-1}=0}^{\infty} \alpha_{1}^{n_{1}-1} \ldots \alpha_{\ell-1}^{n_{\ell-1}-1} \omega\left(\begin{array}{l}
n_{1}, \ldots, n_{\ell-1} \\
b_{1}, \ldots, b_{\ell-1}
\end{array}\right)
\end{array}
$$

and similarly for the second term. The sum $\sim \alpha_{\ell}^{-2}$ is canceled by contributions from the last two lines of eq. (E.3), which we will now turn to

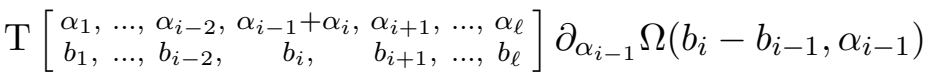

$$
\begin{aligned}
& =\sum_{n_{1}, \ldots, n_{i-2}, n_{i+1}, \ldots, n_{\ell}=0}^{\infty} \alpha_{1}^{n_{1}-1} \ldots \alpha_{i-2}^{n_{i-2}-1} \alpha_{i+1}^{n_{i+1}-1} \ldots \alpha_{\ell}^{n_{\ell}-1}
\end{aligned}
$$

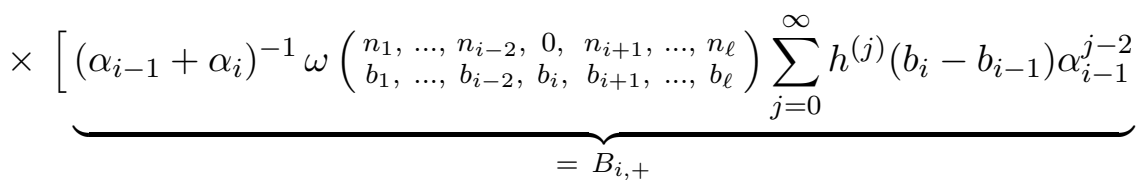

$$
\begin{aligned}
& +\underbrace{\sum_{j, k=0}^{\infty} \sum_{p=0}^{k}\left(\begin{array}{l}
k \\
p
\end{array}\right) \alpha_{i-1}^{k-p+j-2} \alpha_{i}^{p} \omega\left(\begin{array}{c}
n_{1}, \ldots, n_{i-2}, k+1, n_{i+1}, \ldots, n_{\ell} \\
b_{1}, \ldots, b_{i-2}, \quad b_{i}, \\
b_{i+1}, \ldots, b_{\ell}
\end{array}\right) h^{(j)}\left(b_{i}-b_{i-1}\right)}_{=C_{i,+}}]
\end{aligned}
$$

with an analogous definition for $B_{i,-}$ and $C_{i,-}$ relevant to the last term of eq. (E.3)

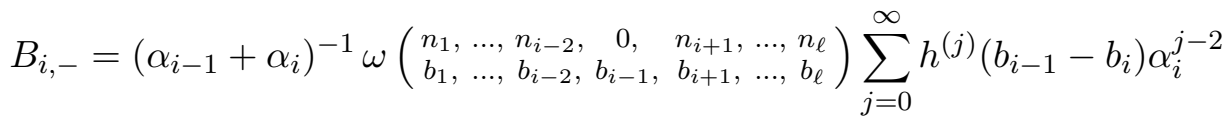

$$
\begin{aligned}
& C_{i,-}=\sum_{j, k=0}^{\infty} \sum_{p=0}^{k}\left(\begin{array}{l}
k \\
p
\end{array}\right) \alpha_{i}^{k-p+j-2} \alpha_{i-1}^{p} \omega\left(\begin{array}{c}
n_{1}, \ldots, n_{i-2}, k+1, \\
b_{1}, \ldots, b_{i-2}, b_{i-1}, b_{i+1}, \ldots, n_{\ell} \\
b_{\ell}
\end{array}\right) h^{(j)}\left(b_{i-1}-b_{i}\right) .
\end{aligned}
$$

In the following the manipulations only affect pairs $\left(\alpha_{i-1}, \alpha_{i}\right)$, hence we will suppress the summation over the other $\alpha$ 's. Since $h^{(0)}(b)=-1$ does not depend on $b$, we can set it to zero for 
the iterated integral

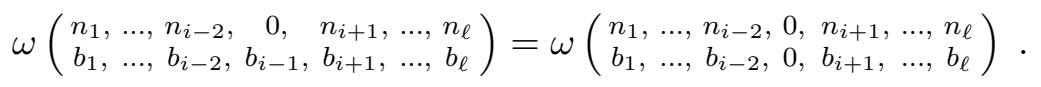

Hence, for the terms not expressible by the binomial law $B_{i, \pm}$, we can use $h^{(0)}=-1, h^{(1)}=0$, $h^{(2)}(b)=h^{(2)}(-b)$ as well as

$$
\left(\alpha_{i-1}^{j}-(-1)^{j} \alpha_{i}^{j}\right)=\left(\alpha_{i-1}+\alpha_{i}\right) \sum_{a=0}^{j-1}(-1)^{j-1-a} \alpha_{i-1}^{a} \alpha_{i}^{j-1-a}, j>0
$$

to obtain

$$
\begin{aligned}
& B_{i,+}-B_{i,-} \\
& =\left(\alpha_{i-1}+\alpha_{i}\right)^{-1} \omega\left(\begin{array}{c}
n_{1}, \ldots, n_{i-2}, 0, n_{i+1}, \ldots, n_{\ell} \\
b_{1}, \ldots, b_{i-2}, 0, b_{i+1}, \ldots, b_{\ell}
\end{array}\right) \sum_{j=0}^{\infty}\left(h^{(j)}\left(b_{i}-b_{i-1}\right) \alpha_{i-1}^{j-2}-h^{(j)}\left(b_{i-1}-b_{i}\right) \alpha_{i}^{j-2}\right) \\
& =\omega\left(\begin{array}{c}
n_{1}, \ldots, n_{i-2}, 0, n_{i+1}, \ldots, n_{\ell} \\
b_{1}, \ldots, b_{i-2}, 0, b_{i+1}, \ldots, b_{\ell}
\end{array}\right)\left(\frac{\alpha_{i}^{-2}-\alpha_{i-1}^{-2}}{\alpha_{i-1}+\alpha_{i}}+\sum_{j=0}^{\infty} h^{(j+3)}\left(b_{i}-b_{i-1}\right) \sum_{a=0}^{j}(-1)^{j-a} \alpha_{i-1}^{a} \alpha_{i}^{j-a}\right) .
\end{aligned}
$$

The singular term $\frac{\alpha_{i}^{-2}-\alpha_{i-1}^{-2}}{\alpha_{i-1}+\alpha_{i}}=\frac{1}{\alpha_{i-1} \alpha_{i}}\left(\frac{1}{\alpha_{i}}-\frac{1}{\alpha_{i-1}}\right)$ will eventually cancel among different $B_{i,+}-B_{i,-}$. The remaining contribution may be rewritten into a form where we can easily read off the coefficient of a given monomial in the $\alpha_{i}$,

$$
\begin{aligned}
& \omega\left(\begin{array}{l}
n_{1}, \ldots, n_{i-2}, 0, n_{i+1}, \ldots, n_{\ell} \\
b_{1}, \ldots, b_{i-2}, 0, b_{i+1}, \ldots, b_{\ell}
\end{array}\right) \sum_{j=0}^{\infty} h^{(j+3)}\left(b_{i}-b_{i-1}\right) \sum_{a=0}^{j}(-1)^{j-a} \alpha_{i-1}^{a} \alpha_{i}^{j-a} \\
& \quad=\omega\left(\begin{array}{l}
n_{1}, \ldots, n_{i-2}, 0, n_{i+1}, \ldots, n_{\ell} \\
b_{1}, \ldots, b_{i-2}, 0, b_{i+1}, \ldots, b_{\ell}
\end{array}\right) \sum_{m, n=0}^{\infty} h^{(m+n+3)}\left(b_{i}-b_{i-1}\right)(-1)^{n} \alpha_{i-1}^{m} \alpha_{i}^{n},
\end{aligned}
$$

which gives rise to the last line of eq. (3.5).

For the contributions $C_{i, \pm}$ we have

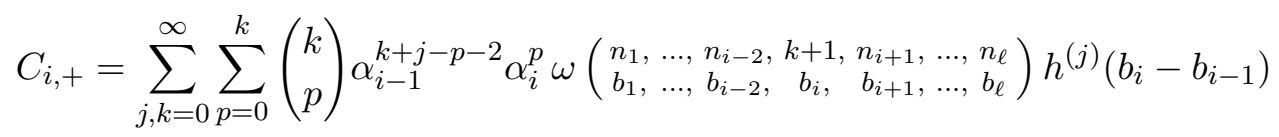

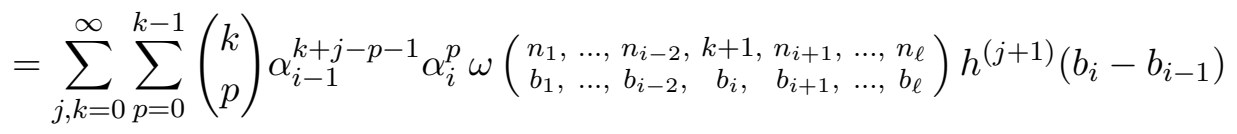

$$
\begin{aligned}
& +\sum_{k=0}^{\infty} \sum_{p=0}^{k}\left(\begin{array}{c}
k+1 \\
p
\end{array}\right) \alpha_{i-1}^{k-p-1} \alpha_{i}^{p} \omega\left(\begin{array}{c}
n_{1}, \ldots, n_{i-2}, \\
b_{1}, \ldots+2, b_{i-2}, \quad b_{i}, \quad b_{i+1}, \ldots, n_{\ell}, \ldots, b_{\ell}
\end{array}\right) h^{(0)}\left(b_{i}-b_{i-1}\right) \\
& +\alpha_{i-1}^{-2} \sum_{a=0}^{\infty} h^{(0)}\left(b_{i}-b_{i-1}\right) \alpha_{i}^{a} \omega\left(\begin{array}{c}
n_{1}, \ldots, n_{i-2}, a+1, n_{i+1}, \ldots, n_{\ell} \\
b_{1}, \ldots, b_{i-2}, \quad b_{i}, \quad b_{i+1}, \ldots, b_{\ell}
\end{array}\right) .
\end{aligned}
$$

The sums proportional to $\alpha_{i-1}^{-2}$ cancel among the corresponding contributions from $C_{i,+}$ and $C_{i-1,-}$. For the cases $i=2$ and $i=r$ it is canceled by the corresponding sums in the last line 
of eq. (E.4). We then find for the remaining part of $C_{i,+}$

$$
\begin{aligned}
& \sum_{j, k=0}^{\infty} \sum_{p=0}^{k-1}\left(\begin{array}{l}
k \\
p
\end{array}\right) \alpha_{i-1}^{k+j-p-1} \alpha_{i}^{p} \omega\left(\begin{array}{c}
n_{1}, \ldots, n_{i-2}, k+1, n_{i+1}, \ldots, n_{\ell} \\
b_{1}, \ldots, b_{i-2}, \quad b_{i}, \quad b_{i+1}, \ldots, b_{\ell}
\end{array}\right) h^{(j+1)}\left(b_{i-1}-b_{i}\right)
\end{aligned}
$$



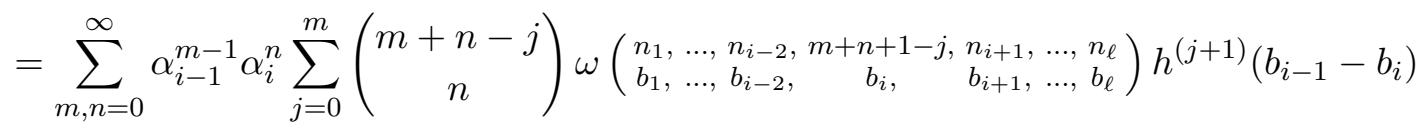

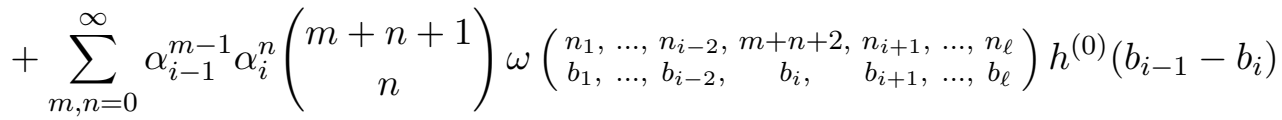

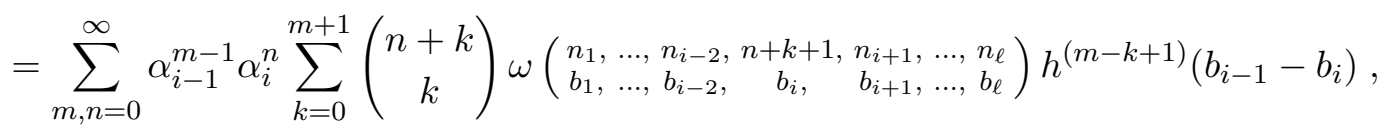

which, in combination with an analogous contribution from $C_{i,-}$, gives rise to the second and third line of eq. (3.5).

\section{F Differential equation for proper rational twists}

The derivation of the differential equation given in appendix E was a priori only valid for generic twists. In this appendix, we provide an argument why it also holds for proper rational twists $b_{i} \in \mathbb{Q}$. The only difference is that in eq. (E.1) we now need to carefully keep track of the effect of deforming the domain of integration $[0,1]$ infinitesimally to obtain $[0,1]_{\varepsilon}$ (cf. figure 3 ). The key point is that the integral along $[0,1]_{\varepsilon}$ is the same as along $[0,1]$ up to terms which vanish in the limit, and the differential equation (E.1) goes through without change.

As in the case of generic twists, it will be convenient to define a generating function

$$
\begin{aligned}
\mathrm{T}^{R}\left[\begin{array}{l}
\alpha_{1}, \ldots, \alpha_{\ell} \\
b_{1}, \ldots, b_{\ell}
\end{array}\right] & =\lim _{\varepsilon \rightarrow 0} \mathrm{~T}_{\varepsilon}^{R}\left[\begin{array}{c}
\alpha_{1}, \ldots, \alpha_{\ell} \\
b_{1}, \ldots, b_{\ell}
\end{array}\right], \\
\mathrm{T}_{\varepsilon}^{R}\left[\begin{array}{l}
\alpha_{1}, \ldots, \alpha_{\ell} \\
b_{1}, \ldots, b_{\ell}
\end{array}\right] & =\int_{0 \leq t_{i} \leq t_{i+1} \leq 1}\left(\gamma_{R}^{*} \Omega\left(z_{1}-b_{1}, \alpha_{1}, \tau\right) \mathrm{d} z_{1}\right) \ldots\left(\gamma_{R}^{*} \Omega\left(z_{\ell}-b_{\ell}, \alpha_{\ell}, \tau\right) \mathrm{d} z_{\ell}\right),
\end{aligned}
$$

where $\gamma_{R}=[0,1]_{\varepsilon}$ and $\gamma_{R}^{*}$ denotes its pullback. Here and throughout this appendix, we will denote the pullback of the coordinate $z_{i}$ along $\gamma_{R}$ by $t_{i}$. We note that in the case where all $b_{i}$ are generic twists, we may pass to the limit $\varepsilon \rightarrow 0$ immediately and integrate along the line $\gamma_{R}(t)=t$, which leads to eq. (3.1). Pulling back $\Omega(z, \alpha, \tau) \mathrm{d} z$ along $\gamma_{R}$, we obtain

$$
\gamma_{R}^{*} \Omega\left(z_{i}-b_{i}, \alpha_{i}, \tau\right) \mathrm{d} z_{i}=\gamma_{R}^{*} \underbrace{e^{-2 \pi i r_{i} \alpha_{i}} F\left(z_{i}-b_{i}, \alpha_{i}, \tau\right) \mathrm{d} z_{i}}_{=\tilde{\Omega}\left(z_{i}-b_{i}, \alpha_{i}, \tau\right) \mathrm{d} z_{i}}+\mathcal{O}(\varepsilon),
$$

since $\operatorname{Im}\left(z_{i}\right)$ is of order $\varepsilon$ on $[0,1]_{\varepsilon}$.

The resulting form $\tilde{\Omega}(z, \alpha, \tau) \mathrm{d} z$ is now meromorphic; therefore the integral over $\tilde{\Omega}(z, \alpha, \tau) \mathrm{d} z$ along any path depends exclusively on its homotopy class. In particular, since the paths $[0,1]_{\varepsilon}$ 
are all homotopic for sufficiently small $\varepsilon$, for every such $\varepsilon$ we get ${ }^{17}$

$$
\begin{aligned}
& \mathrm{T}^{R}\left[\begin{array}{l}
\alpha_{1}, \ldots, \alpha_{\ell} \\
b_{1}, \ldots, b_{\ell}
\end{array}\right]=\lim _{\varepsilon \rightarrow 0} \tilde{\mathrm{T}}_{\varepsilon}^{R}\left[\begin{array}{c}
\alpha_{1}, \ldots, \alpha_{\ell} \\
b_{1}, \ldots, b_{\ell}
\end{array}\right]=\tilde{\mathrm{T}}_{\varepsilon}^{R}\left[\begin{array}{c}
\alpha_{1}, \ldots, \alpha_{\ell} \\
b_{1}, \ldots, b_{\ell}
\end{array}\right], \\
& \tilde{\mathrm{T}}_{\varepsilon}^{R}\left[\begin{array}{l}
\alpha_{1}, \ldots, \alpha_{\ell} \\
b_{1}, \ldots, b_{\ell}
\end{array}\right]=\int_{0 \leq t_{i} \leq t_{i+1} \leq 1}\left(\gamma_{R}^{*} \tilde{\Omega}\left(z_{1}-b_{1}, \alpha_{1}, \tau\right) \mathrm{d} z_{1}\right) \ldots\left(\gamma_{R}^{*} \tilde{\Omega}\left(z_{\ell}-b_{\ell}, \alpha_{\ell}, \tau\right) \mathrm{d} z_{\ell}\right) .
\end{aligned}
$$

Furthermore, we define the intermediate object

$$
\begin{aligned}
\tilde{\mathrm{T}}_{\varepsilon}^{R}\left[\begin{array}{c}
\alpha_{1}, \ldots, \alpha_{i} \\
b_{1}, \ldots, b_{i}
\end{array} ; z_{i+1}\right] & =\int_{0 \leq t_{1} \leq \ldots \leq t_{i} \leq t_{i+1}}\left(\gamma_{R}^{*} \tilde{\Omega}\left(z_{1}-b_{1}, \alpha_{1}, \tau\right) \mathrm{d} z_{1}\right) \ldots\left(\gamma_{R}^{*} \tilde{\Omega}\left(z_{i}-b_{i}, \alpha_{i}, \tau\right) \mathrm{d} z_{i}\right) \\
& =\int_{0}^{t_{i+1}} \gamma_{R}^{*}\left(\tilde{\Omega}\left(z_{i}-b_{i}, \alpha_{i}, \tau\right) \tilde{\mathrm{T}}_{\varepsilon}^{R}\left[\begin{array}{c}
\alpha_{1}, \ldots, \alpha_{i} \\
b_{1}, \ldots, b_{i}
\end{array} ; z_{i}\right] \mathrm{d} z_{i}\right)
\end{aligned}
$$

with $0<t_{i+1}<1$. It satisfies

$$
\partial_{z_{i+1}} \tilde{\mathrm{T}}_{\varepsilon}^{R}\left[\begin{array}{c}
\alpha_{1}, \ldots, \alpha_{i} \\
b_{1}, \ldots, b_{i}
\end{array} ; z_{i+1}\right]=\tilde{\Omega}\left(z_{i+1}-b_{i}, \alpha_{i}, \tau\right) \tilde{\mathrm{T}}_{\varepsilon}^{R}\left[\begin{array}{c}
\alpha_{1}, \ldots, \alpha_{i-1} \\
b_{1}, \ldots, b_{i-1}
\end{array} ; z_{i+1}\right] .
$$

Using the above setup, we now show that all essential aspects of the computation (E.1) in appendix E remain unchanged. Firstly, we compute the $\tau$-derivative of $\gamma_{R}^{*} \tilde{\Omega}$ and obtain

$$
\begin{aligned}
2 \pi i \partial_{\tau} \gamma_{R}^{*}(\tilde{\Omega} & \left.\left(z_{i}-b_{i}, \alpha_{i}, \tau\right) \mathrm{d} z_{i}\right) \\
& =2 \pi i \partial_{\tau}\left(e^{-2 \pi i r_{i} \alpha_{i}} F\left(\gamma_{R}\left(t_{i}\right)-b_{i}, \alpha_{i}, \tau\right) \mathrm{d} \gamma_{R}\left(t_{i}\right)\right) \\
& =e^{-2 \pi i r_{i} \alpha_{i}}\left[\left(-2 \pi i r_{i} \partial_{\gamma_{R}\left(t_{i}\right)}+\partial_{\gamma_{R}\left(t_{i}\right)} \partial_{\alpha_{i}}\right) F\left(\gamma_{R}\left(t_{i}\right)-b_{i}, \alpha_{i}, \tau\right)\right] \mathrm{d} \gamma_{R}\left(t_{i}\right) \\
& =\gamma_{R}^{*}\left(\partial_{z_{i}} \partial_{\alpha_{i}} \tilde{\Omega}\left(z_{i}-b_{i}, \alpha_{i}, \tau\right) \mathrm{d} z_{i}\right),
\end{aligned}
$$

using the mixed heat equation (B.4) for $F(z, \alpha, \tau)$ in the second step. In particular, $\gamma_{R}^{*} \tilde{\Omega}$ itself satisfies a mixed-heat type equation. Secondly, we may exchange the $\tau$-derivative with the integration

$$
\begin{aligned}
2 \pi i \partial_{\tau} \tilde{\mathrm{T}}_{\varepsilon}^{R}\left[\begin{array}{c}
\alpha_{1}, \ldots, \alpha_{\ell} \\
b_{1}, \ldots, b_{\ell}
\end{array}\right]=\sum_{i=1}^{\ell} & \int_{\substack{0 \leq t_{j-1} \leq t_{j} \leq 1 \\
t_{i+1}}} \prod_{j>i}^{\ell}\left(\gamma_{R}^{*} \tilde{\Omega}\left(z_{j}-b_{j}, \alpha_{j}, \tau\right) \mathrm{d} z_{j}\right) \\
& \times \int_{0}^{t_{R}} \gamma_{R}^{*}\left(\left(\partial_{z_{i}} \partial_{\alpha_{i}} \tilde{\Omega}\left(z_{i}-b_{i}, \alpha_{i}, \tau\right)\right) \tilde{\mathrm{T}}_{\varepsilon}^{R}\left[\begin{array}{c}
\alpha_{1}, \ldots, \alpha_{i-1} \\
b_{1}, \ldots, b_{i-1}
\end{array} z_{i}\right] \mathrm{d} z_{i}\right),
\end{aligned}
$$

since the $\tau$-derivative of the integrand is bounded on the domain of integration. Rewriting the $i^{\text {th }}$ integration using integration by parts yields

$$
\begin{aligned}
& \int_{0}^{t_{i+1}} \gamma_{R}^{*}\left(\left(\partial_{z_{i}} \partial_{\alpha_{i}} \tilde{\Omega}\left(z_{i}-b_{i}, \alpha_{i}, \tau\right)\right) \tilde{\mathrm{T}}_{\varepsilon}^{R}\left[\begin{array}{l}
\alpha_{1}, \ldots, \alpha_{i-1} \\
b_{1}, \ldots, b_{i-1} ; z_{i}
\end{array}\right] \mathrm{d} z_{i}\right) \\
& \left.=\gamma_{R}^{*}\left(\partial_{\alpha_{i}} \tilde{\Omega}\left(z_{i+1}-b_{i}, \alpha_{i}, \tau\right)\right) \tilde{\mathrm{T}}_{\varepsilon}^{R}\left[\begin{array}{l}
\alpha_{1}, \ldots, \alpha_{i-1} \\
b_{1}, \ldots, b_{i-1} ; z_{i+1}
\end{array}\right]\right)
\end{aligned}
$$

\footnotetext{
${ }^{17}$ Recall that the same argument has already been used in subsection 2.1 to show that our version of teMZVs for proper rational twists is well-defined (more precisely that the limit in eq. (2.7) exists).
} 


$$
-\int_{0}^{t_{i+1}} \gamma_{R}^{*}\left(\left(\partial_{\alpha_{i}} \tilde{\Omega}\left(z_{i}-b_{i}, \alpha_{i}, \tau\right)\right) \tilde{\Omega}\left(z_{i-1}-b_{i-1}, \alpha_{i-1}, \tau\right) \tilde{\mathrm{T}}_{\varepsilon}^{R}\left[\begin{array}{l}
\alpha_{1}, \ldots, \alpha_{i-2} \\
b_{1}, \ldots, b_{i-2} ; z_{i}
\end{array}\right] \mathrm{d} z_{i}\right),
$$

as in the case of generic twists. Therefore, we may proceed further as in the computation of eq. (E.1) and arrive at the same result simply by virtue of the replacements $\mathrm{T} \rightarrow \mathrm{T}_{\varepsilon}^{R}$ and $\Omega \rightarrow \tilde{\Omega}$.

\section{G Properties of teMZVs}

The purpose of this appendix is to gather constant terms and $q$-expansions of teMZVs as well as selected relations relevant to the one-loop open-string amplitude in section 4 .

\section{G.1 Constant terms for generic twists}

We start by listing a few simple examples for constant terms of teMZVs with generic twists. These constant terms are obtained from eq. (3.18) by comparing the coefficients of words in the non-commutative variables $\operatorname{ad}_{x_{b}}^{n}(y)$ on both sides, see eq. $(3.14)$ for the change of alphabet between the two sides. At length one, specializing eqs. (3.11) and (3.12) to $r=\frac{1}{2}$ yields

$$
\omega_{0}\left(\begin{array}{c}
n \\
\tau / 2
\end{array}\right)=\left\{\begin{array}{cl}
\frac{2^{n-1}-1}{2^{n-2}} \zeta_{n} & : n \text { even } \\
0 & : n \text { odd }
\end{array}\right.
$$

see eq. $(2.24)$ for the $\omega_{0}(\ldots)$ notation. This immediately implies that

$$
\omega_{0}\left(\begin{array}{rr}
\ldots, 2 k-1, \ldots \\
\ldots, & \tau / 2,
\end{array}\right)=0, \quad k \in \mathbb{N}
$$

regardless on the position of the combined letter $\begin{gathered}2 k-1 \\ \tau / 2\end{gathered}$. Similarly, higher-length examples include

$$
\begin{aligned}
& \omega_{0}\left(\begin{array}{cl}
n, 0 \\
\tau / 2,0
\end{array}\right)=\left\{\begin{array}{cl}
\frac{2^{n-1}-1}{2^{n-1} \zeta_{n}} & : n \text { even } \\
0 & : n \text { odd }
\end{array}, \quad \omega_{0}\left(\begin{array}{c}
1, n \\
0, \tau / 2
\end{array}\right)=\left\{\begin{array}{cl}
(-i \pi) \frac{2^{n-1}-1}{2^{n-1}} \zeta_{n} & : n \text { even } \\
0 & : n \text { odd }
\end{array}\right.\right. \\
& \omega_{0}\left(\begin{array}{l}
2,0,0 \\
0,0,0
\end{array}\right)=-\frac{\zeta_{2}}{3}, \quad \omega_{0}\left(\begin{array}{l}
0,1,0,0 \\
0,0,0,0
\end{array}\right)=\frac{3 \zeta_{3}}{4 \pi^{2}}, \quad \omega_{0}\left(\begin{array}{l}
0,3,0,0 \\
0,0,0,0
\end{array}\right)=0 \\
& \omega_{0}\left(\begin{array}{c}
1,0,2 \\
0,0, \tau / 2
\end{array}\right)=-\frac{i \pi^{3}}{24}, \quad \omega_{0}\left(\begin{array}{cc}
0,2,2 \\
0,0, \tau / 2
\end{array}\right)=-\frac{\pi^{4}}{108}, \quad \omega_{0}\left(\begin{array}{ccc}
0, & 1,0, & 2 \\
0,0,0, \tau / 2
\end{array}\right)=\frac{\zeta_{3}}{8},
\end{aligned}
$$

and we obtain the following examples with more general twists $b \in\left(\Lambda_{N}+\Lambda_{N} \tau\right) \backslash \Lambda_{N}^{\times}$:

$$
\begin{aligned}
& \omega_{0}\left(\begin{array}{c}
1 / 2+\tau / 3 \\
1 / 2
\end{array}\right)=-\frac{i \pi}{3}, \\
& \omega_{0}\left(\begin{array}{cc}
1, & 1 \\
\tau / 3, & \tau / 5
\end{array}\right)=-\frac{3}{5} \zeta_{2}, \\
& \omega_{0}\left(\begin{array}{ccc}
2, & 1, & 0,1 \\
\tau / 2, \tau / 4, & 0, & 0
\end{array}\right)=-\frac{i \pi}{48} \zeta_{3}+\frac{5}{8} \zeta_{4}, \\
& \omega_{0}\left(\begin{array}{ccc}
3, & 1,0, & 1 \\
2 \tau / 5, & 0,0, \tau / 4
\end{array}\right)=-\frac{9}{125} \zeta_{2} \zeta_{3} .
\end{aligned}
$$

Given a twist $b=s+r \tau$ with $r \neq 0$, the constant term does not depend on $s$ (cf. eqs. (3.11) and $(3.12))$.

Up to weight five and length three (respectively weight three and length four), we have checked the constant-term procedure for consistency with Fay relations among teMZVs which can be derived along the lines of 10 . As already noted above, the constant-term procedure discussed in subsection 3.2 covers all eMZVs occurring in the one-loop open-string amplitude at 
the orders considered in section 4 . The examples presented here address all teMZVs relevant to the calculations in section 4.3 .

\section{G.2 Constant terms for proper rational twists}

For the proper rational twist $b=\frac{1}{2}$ we arrive at the following examples of constant terms

$$
\begin{aligned}
& \omega_{0}\left(\begin{array}{c}
1 \\
1 / 2
\end{array}\right)=-i \pi, \\
& \omega_{0}\left(\begin{array}{ccc}
2, & 0, & 1 \\
1 / 2, & 0,1 / 2
\end{array}\right)=\frac{i \pi^{3}}{24}-\zeta_{2} \log (2), \\
& \omega_{0}\left(\begin{array}{cc}
0, & 1 \\
0, & 1 / 2
\end{array}\right)=-\frac{i \pi}{2}+\log (2), \\
& \omega_{0}\left(\begin{array}{cc}
1, & 0,0 \\
1 / 2, & 0,0
\end{array}\right)=-\frac{i \pi}{8}-\frac{\log (2)}{2},
\end{aligned}
$$

while twists $b \in \Lambda_{3}^{\times}$give rise to

$$
\begin{aligned}
& \omega_{0}\left(\begin{array}{c}
1 \\
1 / 3
\end{array}\right)=-i \pi, \quad \omega_{0}\left(\begin{array}{cc}
1, & 1 \\
1 / 3, & 2 / 3
\end{array}\right)=i \pi\left(\zeta\left(\begin{array}{c}
1 \\
e^{4 \pi i / 3}
\end{array}\right)-\zeta\left(\begin{array}{c}
1 \\
e^{2 \pi i / 3}
\end{array}\right)\right)-3 \zeta_{2}, \\
& \omega_{0}\left(\begin{array}{c}
1,1,2 \\
0,0,1 / 3
\end{array}\right)=\frac{5}{2} \zeta_{4}, \quad \omega_{0}\left(\begin{array}{cc}
1, & 1,0 \\
1 / 3, & 0,0
\end{array}\right)=-\frac{i \pi}{2} \zeta\left(\begin{array}{c}
1 \\
e^{2 \pi i / 3}
\end{array}\right)+\frac{1}{2} \zeta\left(\begin{array}{c}
2 \\
e^{2 \pi i / 3}
\end{array}\right)-\zeta\left(\begin{array}{c}
1, \\
e^{4 \pi i / 3}, e^{2 \pi i / 3}
\end{array}\right), \\
& \omega_{0}\left(\begin{array}{cc}
1,1,0,1 \\
1 / 3,0,0,0
\end{array}\right)=\frac{i \pi}{4} \zeta_{2}+\zeta_{2} \zeta\left(\begin{array}{c}
1 \\
e^{2 \pi i / 3}
\end{array}\right)+\frac{i \pi}{4} \zeta\left(\begin{array}{c}
2 \\
e^{2 \pi i / 3}
\end{array}\right)+\frac{1}{4} \zeta\left(\begin{array}{c}
3 \\
e^{2 \pi i / 3}
\end{array}\right) \\
& -\frac{i \pi}{2} \zeta\left(\begin{array}{cc}
1, & 1 \\
e^{4 \pi i / 3}, & e^{2 \pi i / 3}
\end{array}\right)-\frac{1}{2} \zeta\left(\begin{array}{cc}
1, & 2 \\
e^{4 \pi i / 3}, & e^{2 \pi i / 3}
\end{array}\right)-\frac{1}{2} \zeta\left(\begin{array}{cc}
2, & 1 \\
e^{4 \pi i / 3}, & e^{2 \pi i / 3}
\end{array}\right),
\end{aligned}
$$

see eq. (D.2) for the definition of cyclotomic MZVs $\zeta\left(\begin{array}{c}n_{1}, n_{2}, \ldots, n_{r} \\ \sigma_{1}, \sigma_{2}, \ldots,\end{array}\right)$.

Again, consistency of the constant-term procedure with Fay relations among teMZVs has been checked up to weights five and three at lengths three and four, respectively.

\section{G.3 teMZV relations for the string amplitude}

The simplification of the string amplitude in section 4.3 requires several relations among teMZVs and eMZVs. The subsequent identities involving teMZVs can be proven by comparing both the constant terms encoded in eq. (3.18) and the $\tau$-derivatives eq. (3.5) of both sides. The relations among eMZVs follow from a combination of Fay and shuffle identities [10] and are listed at https://tools.aei.mpg.de/emzv/, At the second order in $\alpha^{\prime}$, we make use of

$$
\begin{aligned}
& 2 \omega\left(\begin{array}{l}
1,1,0 \\
0,0,0
\end{array}\right)-\omega\left(\begin{array}{l}
1,0 \\
0,0
\end{array}\right)^{2}=\omega\left(\begin{array}{l}
2,0,0 \\
0,0,0
\end{array}\right)+\frac{5 \zeta_{2}}{6} \\
& 2 \omega\left(\begin{array}{ccc}
1, & 1, & 0 \\
\tau / 2, \tau / 2, & 0
\end{array}\right)-\omega\left(\begin{array}{cc}
1, & 0 \\
\tau / 2, & 0
\end{array}\right)^{2}=\omega\left(\begin{array}{c}
2,0,0 \\
0,0,0
\end{array}\right)+\frac{\zeta_{2}}{3},
\end{aligned}
$$

while the simplifications at the order $\alpha^{\prime 3}$ are based on the relations

$$
\begin{aligned}
& \omega\left(\begin{array}{l}
1,0 \\
0,0
\end{array}\right)^{3}-3 \omega\left(\begin{array}{l}
1,0 \\
0,0
\end{array}\right) \omega\left(\begin{array}{l}
1,1,0 \\
0,0,0
\end{array}\right)+3 \omega\left(\begin{array}{l}
1,1,1,0 \\
0,0,0,0
\end{array}\right) \\
& =\frac{1}{6} \omega\left(\begin{array}{l}
0,3,0,0 \\
0,0,0,0
\end{array}\right)-\frac{1}{4} \zeta_{3}-4 \zeta_{2} \omega\left(\begin{array}{l}
0,1,0,0 \\
0,0,0,0
\end{array}\right)
\end{aligned}
$$

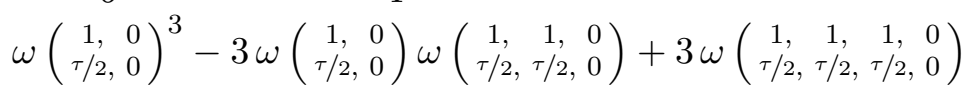

$$
\begin{aligned}
& =\frac{1}{6} \omega\left(\begin{array}{l}
0,3,0,0 \\
0,0,0,0
\end{array}\right)-\frac{1}{4} \zeta_{3}+2 \zeta_{2} \omega\left(\begin{array}{l}
0,1,0,0 \\
0,0,0,0
\end{array}\right) \text {. }
\end{aligned}
$$




\section{G.4 q-expansions for the string amplitude}

The eMZVs seen in the final results eqs. (4.55) and (H.26) for the integrals in the string amplitude have the following $q$-expansions

$$
\begin{aligned}
\omega(0,0,2) & =-\frac{\zeta_{2}}{3}+2 \sum_{n, m=1}^{\infty} \frac{m}{n^{2}} q^{m n} \\
\omega(0,1,0,0) & =\frac{3 \zeta_{3}}{4 \pi^{2}}+\frac{3}{2 \pi^{2}} \sum_{n, m=1}^{\infty} \frac{1}{n^{3}} q^{m n} \\
\omega(0,3,0,0) & =-3 \sum_{n, m=1}^{\infty} \frac{m^{2}}{n^{3}} q^{m n} .
\end{aligned}
$$

These expressions follow from repeatedly integrating the Eisenstein series eq. (3.6) in the $\tau$ derivatives eq. (3.5) of the eMZVs in question. Analogous $q$-expansions for teMZVs with twists $b_{i} \in\{0, \tau / 2\}$ can be determined based on

$$
f^{(k)}(\tau / 2)=\left\{\begin{array}{cl}
\frac{2^{k-1}-1}{2^{k-2} \zeta_{k}-\frac{2(2 \pi i)^{k}}{(k-1) !} \sum_{n, m=1}^{\infty}\left(n-\frac{1}{2}\right)^{k-1} q^{m(n-1 / 2)}} & : k \text { even } \\
0 & : k \text { odd }
\end{array} .\right.
$$

\section{H The non-planar integral along with $\operatorname{Tr}\left(t^{1} t^{2} t^{3}\right) \operatorname{Tr}\left(t^{4}\right)$}

In this appendix, we investigate the low-energy expansion of the integral eq. (4.6) associated with the color factor $\operatorname{Tr}\left(t^{1} t^{2} t^{3}\right) \operatorname{Tr}\left(t^{4}\right)$ in the one-loop four-point open-string amplitude. The representations eqs. (4.12) and (4.13) of the Green functions allow to cast the integral into the form

$$
I_{123 \mid 4}(q)=\int_{123}^{4} \prod_{1 \leq i<j}^{3} \sum_{n_{i j}=0}^{\infty} \frac{\left(s_{i j} \Gamma\left(\begin{array}{c}
1 \\
0
\end{array} ; x_{i j}\right)\right)^{n_{i j}}}{n_{i j} !} \prod_{j=1}^{3} \sum_{n_{j 4}=0}^{\infty} \frac{\left(s_{j 4} \Gamma\left(\begin{array}{c}
1 \\
\tau / 2
\end{array} ; x_{j 4}\right)\right)^{n_{j 4}}}{n_{j 4} !}
$$

analogous to eq. (4.23), where $c(q)$ cancels by momentum conservation eq. $(\overline{4.5})$, and the integration measure is defined by

$$
\begin{aligned}
\int_{123}^{4} & =\int_{0}^{1} \mathrm{~d} x_{4} \int_{0}^{1} \mathrm{~d} x_{3} \int_{0}^{x_{3}} \mathrm{~d} x_{2} \int_{0}^{x_{2}} \mathrm{~d} x_{1} \delta\left(x_{1}\right) \\
& =\int_{0}^{1} \mathrm{~d} x_{4} \delta\left(x_{4}\right)\left(\int_{0}^{1} \mathrm{~d} x_{3} \int_{0}^{x_{3}} \mathrm{~d} x_{2} \int_{0}^{x_{2}} \mathrm{~d} x_{1}+\operatorname{cyc}\left(x_{1}, x_{2}, x_{3}\right)\right) .
\end{aligned}
$$

One can check through the change of variables $x_{i} \rightarrow 1-x_{i}$ and symmetry properties of the Green function that the measure $\int_{132}^{4}$ with $x_{2}$ and $x_{3}$ interchanged yields the same result for the integral eq. (H.1). Hence, one can equivalently employ the simpler measure eq. (4.22) with $x_{i} \in[0,1]$ tailored to the color structure $\operatorname{Tr}\left(t^{1} t^{2}\right) \operatorname{Tr}\left(t^{3} t^{4}\right)$ and rewrite

$$
I_{123 \mid 4}(q)=\frac{1}{2} \int_{12}^{34} \prod_{1 \leq i<j}^{3} \sum_{n_{i j}=0}^{\infty} \frac{\left(s_{i j} \Gamma\left(\begin{array}{c}
1 \\
0
\end{array} ; x_{i j}\right)\right)^{n_{i j}}}{n_{i j} !} \prod_{j=1}^{3} \sum_{n_{j 4}=0}^{\infty} \frac{\left(s_{j 4} \Gamma\left(\begin{array}{c}
1 \\
\tau / 2
\end{array} ; x_{j 4}\right)\right)^{n_{j 4}}}{n_{j 4} !} .
$$

In the remainder of this appendix, we will follow the steps of subsection 4.3 to expand the integral $I_{123 \mid 4}(q)$ to the order $\alpha^{\prime 3}$, where the representation eq. (H.3) is most convenient for practical purposes. While the elliptic iterated integrals in this expansion lead to teMZVs for 
each monomial in $s_{i j}$, the final results for the orders $\alpha^{\prime \leq 3}$ turn out to comprise eMZVs only.

\section{H.1 Structure of the leading orders $\alpha^{\prime \leq 3}$}

We start by classifying the inequivalent integrals w.r.t. the cycle structure of $\operatorname{Tr}\left(t^{1} t^{2} t^{3}\right) \operatorname{Tr}\left(t^{4}\right)$ which occur at the orders $\alpha^{\prime \leq 3}$ of eq. (H.1): With the shorthands $P_{i j}=P\left(x_{i j}\right)$ and

$$
\hat{Q}_{i j}=Q\left(x_{i j}\right)-c(q)=\Gamma\left(\underset{\tau / 2}{1} ; x_{i j}\right),
$$

we have two inequivalent cases at the first order,

$$
e_{1}^{1}=\int_{123}^{4} P_{12}, \quad e_{2}^{1}=\int_{123}^{4} \hat{Q}_{14},
$$

six cases at the second order,

$$
\begin{aligned}
& e_{1}^{2}=\frac{1}{2} \int_{123}^{4} P_{12}^{2}, \quad e_{3}^{2}=\int_{123}^{4} P_{12} P_{13}, \quad e_{5}^{2}=\int_{123}^{4} P_{12} \hat{Q}_{34} \\
& e_{2}^{2}=\frac{1}{2} \int_{123}^{4} \hat{Q}_{14}^{2}, \quad e_{4}^{2}=\int_{123}^{4} \hat{Q}_{14} \hat{Q}_{24}, \quad e_{6}^{2}=\int_{123}^{4} P_{12} \hat{Q}_{14},
\end{aligned}
$$

and fourteen cases at the third order:

$$
\begin{aligned}
& e_{1}^{3}=\frac{1}{6} \int_{123}^{4} P_{12}^{3} \\
& e_{6}^{3}=\frac{1}{2} \int_{123}^{4} \hat{Q}_{14}^{2} \hat{Q}_{24}, \quad e_{11}^{3}=\int_{123}^{4} P_{12} P_{13} \hat{Q}_{14} \\
& e_{2}^{3}=\frac{1}{6} \int_{123}^{4} \hat{Q}_{14}^{3}, \quad e_{7}^{3}=\frac{1}{2} \int_{123}^{4} P_{12}^{2} \hat{Q}_{14}, \quad e_{12}^{3}=\int_{123}^{4} P_{12} P_{13} \hat{Q}_{24} \\
& e_{3}^{3}=\frac{1}{2} \int_{123}^{4} P_{12}^{2} P_{13}, \quad e_{8}^{3}=\frac{1}{2} \int_{123}^{4} P_{12}^{2} \hat{Q}_{34}, \quad e_{13}^{3}=\int_{123}^{4} P_{12} \hat{Q}_{14} \hat{Q}_{24} \\
& e_{4}^{3}=\int_{123}^{4} P_{12} P_{13} P_{23}, \quad e_{9}^{3}=\frac{1}{2} \int_{123}^{4} P_{12} \hat{Q}_{14}^{2}, \quad e_{14}^{3}=\int_{123}^{4} P_{12} \hat{Q}_{14} \hat{Q}_{34} \\
& e_{5}^{3}=\int_{123}^{4} \hat{Q}_{14} \hat{Q}_{24} \hat{Q}_{34}, \quad e_{10}^{3}=\frac{1}{2} \int_{123}^{4} P_{12} \hat{Q}_{34}^{2} \text {. }
\end{aligned}
$$

\section{H.2 teMZVs at orders $\alpha^{\prime \leq 3}$}

As a next step, we evaluate the above $e_{i}^{i}$ in terms of teMZVs, using the equivalence of the measures $\int_{123}^{4}$ and $\frac{1}{2} \int_{12}^{34}$ noted in eqs. (H.1) and (H.3). By largely recycling the results of section 4.3, we obtain the following expressions at the first order,

$$
e_{1}^{1}=\frac{1}{2} d_{1}^{1}=\frac{1}{2} \omega\left(\begin{array}{c}
1,0 \\
0,0
\end{array}\right), \quad e_{2}^{1}=\left.\frac{1}{2} d_{2}^{1}\right|_{c(q) \rightarrow 0}=\frac{1}{2} \omega\left(\begin{array}{cc}
1, & 0 \\
\tau / 2,0 & 0
\end{array}\right),
$$

the following ones at the second order,

$$
\begin{aligned}
& e_{1}^{2}=\left.\frac{1}{2} d_{1}^{2}\right|_{c(q) \rightarrow 0}=\frac{1}{2} \omega\left(\begin{array}{cc}
1,1,0 \\
0,0,0
\end{array}\right), \quad e_{3}^{2}=\left.\frac{1}{2} d_{4}^{2}\right|_{c(q) \rightarrow 0}=\frac{1}{2} \omega\left(\begin{array}{cc}
1,0 \\
0,0
\end{array}\right)^{2} \\
& e_{2}^{2}=\left.\frac{1}{2} d_{2}^{2}\right|_{c(q) \rightarrow 0}=\frac{1}{2} \omega\left(\begin{array}{ccc}
1, & 1,0 \\
\tau / 2, \tau / 2,0 & 0
\end{array}\right), \quad e_{4}^{2}=\left.\frac{1}{2} d_{5}^{2}\right|_{c(q) \rightarrow 0}=\frac{1}{2} \omega\left(\begin{array}{cc}
1, & 0 \\
\tau / 2, & 0
\end{array}\right)^{2} \\
& e_{5}^{2}=\left.\frac{1}{2} d_{3}^{2}\right|_{c(q) \rightarrow 0}=\frac{1}{2} \omega\left(\begin{array}{c}
1,0 \\
0,0
\end{array}\right) \omega\left(\begin{array}{cc}
1, & 0 \\
\tau / 2,0 & 0
\end{array}\right)=e_{6}^{2},
\end{aligned}
$$


and the following ones at the third order:

$$
\begin{aligned}
& e_{1}^{3}=\frac{1}{2} d_{1}^{3}=\frac{1}{2} \omega\left(\begin{array}{l}
1,1,1,0 \\
0,0,0,0
\end{array}\right)
\end{aligned}
$$

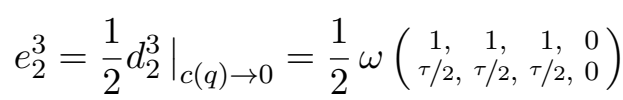

$$
\begin{aligned}
& e_{3}^{3}=\frac{1}{2} d_{5}^{3}=\frac{1}{2} \omega\left(\begin{array}{l}
1,1,0 \\
0,0,0
\end{array}\right) \omega\left(\begin{array}{l}
1,0 \\
0,0
\end{array}\right) \\
& e_{4}^{3}=\frac{1}{2} \omega\left(\begin{array}{l}
1,0 \\
0,0
\end{array}\right)^{3}-\zeta_{2} \omega\left(\begin{array}{l}
0,1,0,0 \\
0,0,0,0
\end{array}\right)+\frac{1}{6} \omega\left(\begin{array}{l}
0,3,0,0 \\
0,0,0,0
\end{array}\right) \\
& e_{5}^{3}=\left.\frac{1}{2} d_{12}^{3}\right|_{c(q) \rightarrow 0}=\frac{1}{2} \omega\left(\begin{array}{rr}
1, & 0 \\
\tau / 2, & 0
\end{array}\right)^{3} \\
& e_{6}^{3}=\left.\frac{1}{2} d_{7}^{3}\right|_{c(q) \rightarrow 0}=\frac{1}{2} \omega\left(\begin{array}{ccc}
1, & 1, & 0 \\
\tau / 2, \tau / 2, & 0
\end{array}\right) \omega\left(\begin{array}{cc}
1, & 0 \\
\tau / 2, & 0
\end{array}\right) \\
& e_{7}^{3}=\left.\frac{1}{2} d_{3}^{3}\right|_{c(q) \rightarrow 0}=\frac{1}{2} \omega\left(\begin{array}{cc}
1,1,0 \\
0,0,0
\end{array}\right) \omega\left(\begin{array}{cc}
1, & 0 \\
\tau / 2,0 & 0
\end{array}\right)=e_{8}^{3} \\
& e_{9}^{3}=\left.\frac{1}{2} d_{4}^{3}\right|_{c(q) \rightarrow 0}=\frac{1}{2} \omega\left(\begin{array}{ccc}
1, & 1, & 0 \\
\tau / 2, \tau / 2, & 0
\end{array}\right) \omega\left(\begin{array}{l}
1,0 \\
0,0
\end{array}\right)=e_{10}^{3} \\
& e_{11}^{3}=\left.\frac{1}{2} d_{8}^{3}\right|_{c(q) \rightarrow 0}=\frac{1}{2} \omega\left(\begin{array}{l}
1,0 \\
0,0
\end{array}\right)^{2} \omega\left(\begin{array}{cc}
1, & 0 \\
\tau / 2,0 & 0
\end{array}\right)=e_{12}^{3} \\
& e_{13}^{3}=\left.\frac{1}{2} d_{11}^{3}\right|_{c(q) \rightarrow 0}=\frac{1}{2} \omega\left(\begin{array}{cc}
1, & 0 \\
\tau / 2, & 0
\end{array}\right)^{2} \omega\left(\begin{array}{l}
1,0 \\
0,0
\end{array}\right)+\frac{1}{6} \omega\left(\begin{array}{lll}
0,3, & 0,0 \\
0, & 0,0,0
\end{array}\right) \\
& e_{14}^{3}=\left.\frac{1}{2} d_{9}^{3}\right|_{c(q) \rightarrow 0}=\frac{1}{2} \omega\left(\begin{array}{cc}
1, & 0 \\
\tau / 2,0 & 0
\end{array}\right)^{2} \omega\left(\begin{array}{c}
1,0 \\
0,0
\end{array}\right) \text {. }
\end{aligned}
$$

Expressions for $d_{11}^{3}$ (cf. eq. $\left.(4.45)\right)$ and $e_{4}^{3}$ are derived in appendix I.

\section{H.3 Assembling the orders $\alpha^{\prime \leq 3}$}

Similar to eq. (4.47), momentum conservation ${ }^{18}$ leaves the following contributions to the integral eq. (H.1) at the orders $\alpha^{\prime \leq 3}$,

$$
\begin{aligned}
I_{123 \mid 4}(q)=\frac{1}{2} & +\left(s_{12}^{2}+s_{12} s_{23}+s_{23}^{2}\right)\left(2 e_{1}^{2}+2 e_{2}^{2}-e_{3}^{2}-e_{4}^{2}\right) \\
& +s_{12} s_{23}\left(s_{12}+s_{23}\right)\left(-3 e_{1}^{3}-3 e_{2}^{3}+3 e_{3}^{3}-e_{4}^{3}-e_{5}^{3}+3 e_{6}^{3}-3 e_{13}^{3}+3 e_{14}^{3}\right)+\mathcal{O}\left(\alpha^{\prime 4}\right),
\end{aligned}
$$

in agreement with the $\alpha^{\prime \leq 1}$ results of ref. 31 . The combinations of $e_{j}^{i}$ can be expressed in terms of eMZVs

$$
\begin{aligned}
\left.I_{123 \mid 4}(q)\right|_{s_{12}^{2}+s_{12} s_{23}+s_{23}^{2}} & =\omega\left(\begin{array}{ll}
1, & 1,0 \\
0, & 0,0
\end{array}\right)-\frac{1}{2} \omega\left(\begin{array}{c}
1,0 \\
0,0
\end{array}\right)^{2}+\omega\left(\begin{array}{ccc}
1, & 1, & 0 \\
\tau / 2, \tau / 2, & 0
\end{array}\right)-\frac{1}{2} \omega\left(\begin{array}{cc}
1, & 0 \\
\tau / 2,0 & 0
\end{array}\right)^{2} \\
& =\frac{7 \zeta_{2}}{12}+\omega(0,0,2) \\
\left.I_{123 \mid 4}(q)\right|_{s_{12} s_{23}\left(s_{12}+s_{23}\right)} & =\frac{3}{2} \omega\left(\begin{array}{l}
1,0 \\
0,0
\end{array}\right) \omega\left(\begin{array}{c}
1,1,0 \\
0,0,0
\end{array}\right)-\frac{3}{2} \omega\left(\begin{array}{c}
1,1,1,0 \\
0,0,0,0
\end{array}\right)-\frac{1}{2} \omega\left(\begin{array}{l}
1,0 \\
0,0
\end{array}\right)^{3}
\end{aligned}
$$

\footnotetext{
${ }^{18}$ We have already exploited the equalities $e_{5}^{2}=e_{6}^{2}, \quad e_{7}^{3}=e_{8}^{3}, \quad e_{9}^{3}=e_{10}^{3}$ and $e_{11}^{3}=e_{12}^{3}$ in simplifying $I_{123 \mid 4}(q)$ to the expression in eq. (H.23).
} 


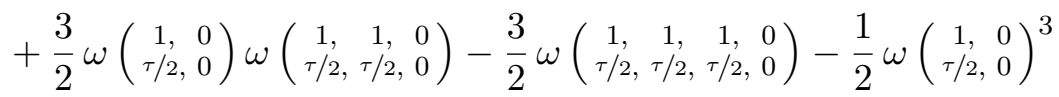

$$
\begin{aligned}
& +\zeta_{2} \omega(0,1,0,0)-\frac{2}{3} \omega(0,3,0,0) \\
& =2 \zeta_{2} \omega(0,1,0,0)-\frac{5}{6} \omega(0,3,0,0)+\frac{\zeta_{3}}{4},
\end{aligned}
$$

where we have used the teMZV relations of appendix G.3.

\section{H.4 Summary of the orders $\alpha^{\prime \leq 3}$}

Once we insert the simplified expressions eqs. (H.24) and (H.25) for the combinations of $e_{j}^{i}$, the leading low-energy orders of the integral eq. (H.23) boil down to the following eMZVs:

$$
\begin{aligned}
I_{123 \mid 4}(q) & =\frac{1}{2}+\left(s_{12}^{2}+s_{12} s_{23}+s_{23}^{2}\right)\left(\frac{7 \zeta_{2}}{12}+\omega(0,0,2)\right) \\
& +s_{12} s_{23}\left(s_{12}+s_{23}\right)\left(2 \zeta_{2} \omega(0,1,0,0)-\frac{5}{6} \omega(0,3,0,0)+\frac{\zeta_{3}}{4}\right)+\mathcal{O}\left(\alpha^{\prime 4}\right) .
\end{aligned}
$$

From the constant terms of the eMZVs gathered in eq. (G.3), our result eq. (H.26) is consistent with the tree-level expression

$$
\begin{aligned}
I_{123 \mid 4}(q) & =-\frac{1}{\pi^{2}}\left[\frac{\Gamma\left(s_{12}\right) \Gamma\left(s_{23}\right)}{\Gamma\left(1+s_{12}+s_{23}\right)}+\operatorname{cyc}(1,2,3)\right]+\mathcal{O}(q) \\
& =\frac{1}{2}+\frac{1}{4} \zeta_{2}\left(s_{12}^{2}+s_{12} s_{23}+s_{23}^{2}\right)+\frac{1}{2} \zeta_{3} s_{12} s_{23}\left(s_{12}+s_{23}\right)+\mathcal{O}\left(q, \alpha^{\prime 4}\right),
\end{aligned}
$$

which is known to arise at the $q^{0}$ order of the integral $I_{123 \mid 4}(q)[32$.

\section{Sample integrals in non-planar string amplitudes}

In this appendix, we will derive the results eqs. $(4.45)$ and (H.15) for $d_{11}^{3}$ and $e_{4}^{3}$, respectively. These integrals are the most difficult cases at the orders $\alpha^{\prime \leq 3}$ because the Green functions form closed subcycles such as $P_{i j} P_{i k} P_{j k}$ and $P_{i j} Q_{i k} Q_{j k}$. Identities between elliptic iterated integrals will be seen to yield answers in terms of teMZVs, and the extensions of these manipulations to all weights and lengths 30 guarantee that each term in the low-energy expansion of the integrals eqs. (4.23) and (H.1) can be expressed in terms of teMZVs.

\section{I.1 The $d_{11}^{3}$ integral from $P_{i j} Q_{i k} Q_{j k}$}

The contributions to $d_{11}^{3}$ with at least one factor of $c(q)$ are equivalent to those in $d_{9}^{3}$, so it is sufficient to study

$$
\begin{aligned}
\left.\int_{12}^{34} P_{34} Q_{13} Q_{14}\right|_{c(q) \rightarrow 0} & =2 \int_{0}^{1} \mathrm{~d} x_{4} \int_{0}^{x_{4}} \mathrm{~d} x_{3} \Gamma\left(\begin{array}{c}
1 \\
\tau / 2
\end{array} ; x_{3}\right) \Gamma\left(\begin{array}{c}
1 \\
\tau / 2
\end{array} ; x_{4}\right)\left(-\int_{x_{3}}^{x_{4}} \mathrm{~d} u f^{(1)}\left(u-x_{4}\right)\right) \\
& =-2 \int_{0}^{1} \mathrm{~d} x_{4} \Gamma\left(\begin{array}{c}
1 \\
\tau / 2
\end{array} ; x_{4}\right) \int_{0}^{x_{4}} \mathrm{~d} u f^{(1)}\left(u-x_{4}\right) \int_{0}^{u} \mathrm{~d} x_{3} \Gamma\left(\begin{array}{c}
1 \\
\tau / 2
\end{array} ; x_{3}\right) \\
& =-2 \int_{0}^{1} \mathrm{~d} x_{4} \Gamma\left(\begin{array}{ccc}
1 \\
\tau / 2
\end{array} ; x_{4}\right) \Gamma\left(\begin{array}{ccc}
1 & 0 & 1 \\
x_{4} & 0 & \tau / 2
\end{array} ; x_{4}\right) .
\end{aligned}
$$

In the first step, we have exploited that the integration regions with $0 \leq x_{3} \leq x_{4} \leq 1$ and $0 \leq x_{4} \leq x_{3} \leq 1$ yield the same contributions by the symmetry of the integrand $P_{34} Q_{13} Q_{14}$ 
under exchange of $x_{3}$ and $x_{4}$. Moreover, we have used the integral representation $P_{34}=$ $-\int_{x_{3}}^{x_{4}} \mathrm{~d} u f^{(1)}\left(u-x_{4}\right)$ of the Green function, reparametrized the integration domain with $0 \leq$ $x_{3} \leq u \leq x_{4}$ and applied the definition eq. (2.1) of elliptic iterated integrals.

The elliptic iterated integral $\Gamma\left(\begin{array}{ccc}1 & 0 & 1 \\ x_{4} & 0 & \tau / 2\end{array} ; x_{4}\right)$ in the last line of eq. (I.1) is not yet suitable for integration over $x_{4}$ in its present form due to the twofold appearance of the integration variable. As explained in 30 , Fay relations among the weighting functions allow to derive a differential equation in $x_{4}$ whose integration yields the alternative representation

$$
\begin{gathered}
\Gamma\left(\begin{array}{ccc}
1 & 0 & 1 \\
x_{4} & 0 & \tau / 2
\end{array} ; x_{4}\right)=\Gamma\left(\begin{array}{ccc}
0 & 0 & 2 \\
0 & 0 & 0
\end{array} ; x_{4}\right)+\Gamma\left(\begin{array}{ccc}
0 & 0 & 2 \\
0 & 0 & \tau / 2
\end{array} ; x_{4}\right)+\Gamma\left(\begin{array}{ccc}
0 & 2 & 0 \\
0 & \tau / 2 & 0
\end{array} ; x_{4}\right) \\
-\Gamma\left(\begin{array}{ccc}
0 & 1 & 1 \\
0 & \tau / 2 & \tau / 2
\end{array} ; x_{4}\right)-\Gamma\left(\begin{array}{ccc}
0 & 1 & 1 \\
0 & \tau / 2 & 0
\end{array} ; x_{4}\right) .
\end{gathered}
$$

After shuffle multiplication with $\Gamma\left(\underset{\tau / 2}{1} ; x_{4}\right)$, the integral over $x_{4}$ in eq. (I.1) can be readily performed, e.g.

$$
\begin{aligned}
& \int_{0}^{1} \mathrm{~d} x_{4} \Gamma\left(\begin{array}{c}
1 \\
\tau / 2
\end{array} ; x_{4}\right) \Gamma\left(\begin{array}{ccc}
0 & 1 & 1 \\
0 & \tau / 2 & 0
\end{array} ; x_{4}\right) \\
& =\int_{0}^{1} \mathrm{~d} x_{4}\left[\Gamma\left(\begin{array}{cccc}
1 & 0 & 1 & 1 \\
\tau / 2 & 0 & \tau / 2 & 0
\end{array} ; x_{4}\right)+2 \Gamma\left(\begin{array}{cccc}
0 & 1 & 1 & 1 \\
0 & \tau / 2 & \tau / 2 & 0
\end{array} ; x_{4}\right)+\Gamma\left(\begin{array}{cccc}
0 & 1 & 1 & 1 \\
0 & \tau / 2 & 0 & \tau / 2
\end{array} ; x_{4}\right)\right] \\
& =\Gamma\left(\begin{array}{ccccc}
0 & 1 & 0 & 1 & 1 \\
0 & \tau / 2 & 0 & \tau / 2 & 0
\end{array} ;\right)+2 \Gamma\left(\begin{array}{ccccc}
0 & 0 & 1 & 1 & 1 \\
0 & 0 & \tau / 2 & \tau / 2 & 0
\end{array} ; 1\right)+\Gamma\left(\begin{array}{ccccc}
0 & 0 & 1 & 1 & 1 \\
0 & 0 & \tau / 2 & 0 & \tau / 2
\end{array} ; 1\right)
\end{aligned}
$$

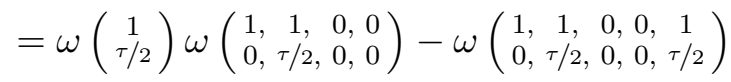

for the rightmost term in eq. (I.2). The shuffle operation in the last step of eq. (I.3) together with $\omega\left(\begin{array}{c}1 \\ \tau / 2\end{array}\right)=0$ reduces the number of terms and leads to the following end result

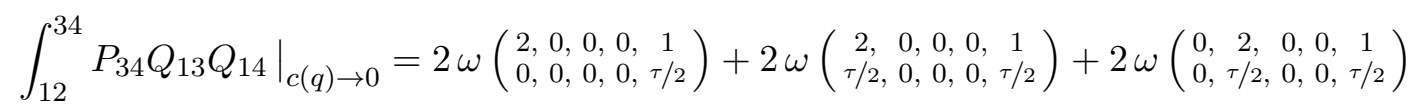



Finally, the expression for $d_{11}^{3}$ in eq. (4.45) results from the eMZV relation

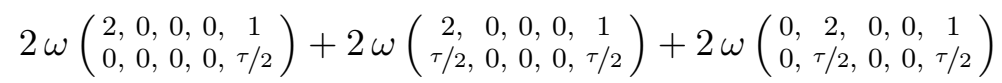

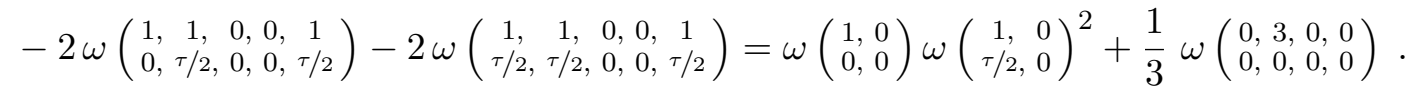

\section{I.2 The $e_{4}^{3}$ integral from $\boldsymbol{P}_{i j} \boldsymbol{P}_{i k} \boldsymbol{P}_{j k}$}

A similar strategy applies to $e_{4}^{3}$ in eq. (H.7),

$$
\begin{aligned}
\int_{123}^{4} P_{12} P_{13} P_{23} & =\int_{0}^{1} \mathrm{~d} x_{3} \int_{0}^{x_{3}} \mathrm{~d} x_{2} \Gamma\left(\begin{array}{c}
1 \\
0
\end{array} x_{2}\right) \Gamma\left(\begin{array}{c}
1 \\
0
\end{array} x_{3}\right)\left(-\int_{x_{2}}^{x_{3}} \mathrm{~d} u f^{(1)}\left(u-x_{3}\right)\right) \\
& =-\int_{0}^{1} \mathrm{~d} x_{3} \Gamma\left(\begin{array}{c}
1 \\
0
\end{array} ; x_{3}\right) \Gamma\left(\begin{array}{ccc}
1 & 0 & 1 \\
x_{3} & 0 & 0
\end{array} x_{3}\right),
\end{aligned}
$$

where the relevant identity among elliptic iterated integrals reads

$$
\Gamma\left(\begin{array}{ccc}
1 & 0 & 1 \\
x_{3} & 0 & 0
\end{array} x_{3}\right)=2 \Gamma\left(\begin{array}{ccc}
0 & 0 & 2 \\
0 & 0 & 0
\end{array} x_{3}\right)+\Gamma\left(\begin{array}{ccc}
0 & 2 & 0 \\
0 & 0 & 0
\end{array} x_{3}\right)-2 \Gamma\left(\begin{array}{ccc}
0 & 1 & 1 \\
0 & 0 & 0
\end{array} ; x_{3}\right)+\zeta_{2} \Gamma\left(\begin{array}{c}
0 \\
0
\end{array} ; x_{3}\right) .
$$


Note the extra term $\zeta_{2} \Gamma\left(\begin{array}{l}0 \\ 0\end{array} ; x_{3}\right)$ in comparison to the analogous identity eq. (I.2). Upon insertion into eq. (I.6), we obtain

$$
\int_{123}^{4} P_{12} P_{13} P_{23}=2 \omega(2,0,0,0,1)+\omega(0,2,0,0,1)-2 \omega(1,1,0,0,1)-\frac{1}{2} \zeta_{2} \omega(1,0),
$$

which translates into the expression eq. (H.15) for $e_{4}^{3}$ by the eMZV relation

$$
\begin{gathered}
2 \omega(2,0,0,0,1)+\omega(0,2,0,0,1)-2 \omega(1,1,0,0,1)-\frac{1}{2} \zeta_{2} \omega(1,0) \\
=\frac{1}{2} \omega(1,0)^{3}-\zeta_{2} \omega(0,1,0,0)+\frac{1}{6} \omega(0,3,0,0) .
\end{gathered}
$$

\section{J Some all-order contributions}

For certain contributions to the integral eq. (4.3) in the non-planar open-string amplitude, closed-form expressions in terms of teMZVs can be given to all orders in the $\alpha^{\prime}$-expansion. In the context and notation of subsection 4.3 , we find

$$
\begin{aligned}
& d_{n ; 1}=\frac{1}{n !} \int_{12}^{34} P\left(x_{12}\right)^{n}=\frac{1}{n !} \int_{0}^{1} \mathrm{~d} x_{2} \prod_{i=1}^{n} \int_{0}^{x_{2}} \mathrm{~d} y_{i} f^{(1)}\left(y_{i}\right)=\omega(\underbrace{1, \ldots, 1,0}_{n \text { times }} \begin{array}{l}
1, \ldots, 0 \\
0,0
\end{array}) \\
& d_{n ; 2}=\frac{1}{n !} \int_{12}^{34} Q\left(x_{13}\right)^{n}=\frac{1}{n !} \int_{12}^{34}\left(\sum_{r=0}^{n}\left(\begin{array}{l}
n \\
r
\end{array}\right) c(q)^{n-r} \Gamma\left(\underset{\tau / 2}{1} ; x_{3}\right)^{r}\right)
\end{aligned}
$$

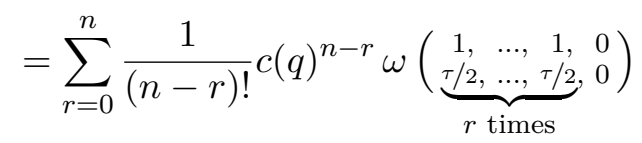

$$
\begin{aligned}
& d_{n, m ; 1}=\frac{1}{n ! m !} \int_{12}^{34} P\left(x_{12}\right)^{n} P\left(x_{34}\right)^{m}=d_{n ; 1} d_{m ; 1}
\end{aligned}
$$

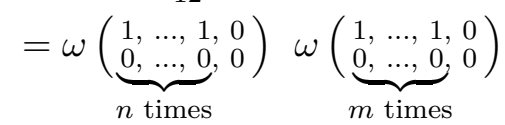

$$
\begin{aligned}
& d_{n, m ; 2}=\frac{1}{n ! m !} \int_{12}^{34} P\left(x_{12}\right)^{n} Q\left(x_{13}\right)^{m}=d_{n ; 1} d_{m ; 2}
\end{aligned}
$$

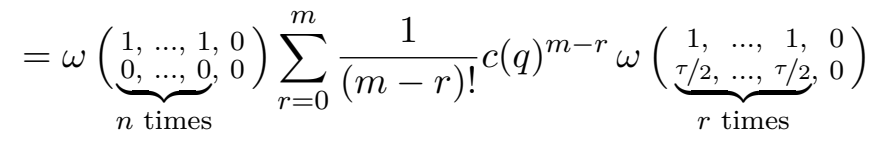

$$
\begin{aligned}
& d_{n, m ; 3}=\frac{1}{n ! m !} \int_{12}^{34} Q\left(x_{13}\right)^{n} Q\left(x_{14}\right)^{m}=d_{n ; 2} d_{m ; 2} \\
& d_{n, m, p ; 1}=\frac{1}{n ! m ! p !} \int_{12}^{34} P\left(x_{12}\right)^{n} P\left(x_{34}\right)^{m} Q\left(x_{13}\right)^{p}=d_{n ; 1} d_{m, p ; 2}=d_{n ; 1} d_{m ; 1} d_{p ; 2} \\
& d_{n, m, p ; 2}=\frac{1}{n ! m ! p !} \int_{12}^{34} P\left(x_{12}\right)^{n} Q\left(x_{13}\right)^{m} Q\left(x_{14}\right)^{p}=d_{n ; 1} d_{m, p ; 3}=d_{n ; 1} d_{m ; 2} d_{p ; 2} .
\end{aligned}
$$

\section{References}

[1] M. E. Hoffman, "Multiple harmonic series", Pacific J. Math. 152, 275 (1992), http://projecteuclid.org/euclid.pjm/1102636166 
[2] D. Zagier, "Values of zeta functions and their applications", in: "First European Congress of Mathematics, Vol. II (Paris, 1992)", Birkhäuser, Basel (1994), 497-512p.

[3] A. Goncharov, "Multiple polylogarithms and mixed Tate motives", math/0103059

[4] A. Levin and G. Racinet, "Towards multiple elliptic polylogarithms", math/0703237

[5] D. Calaque, B. Enriquez and P. Etingof, "Universal KZB equations: the elliptic case", math/0702670, in: "Algebra, arithmetic, and geometry: in honor of Yu. I. Manin. Vol. I", Birkhäuser Boston, Inc., Boston, MA (2009), 165-266p.

[6] F. Brown and A. Levin, "Multiple elliptic polylogarithms", arxiv:1110.6917v2

[7] B. Enriquez, "Elliptic associators", Selecta Math. (N.S.) 20, 491 (2014)

[8] B. Enriquez, "Analogues elliptiques des nombres multizétas", Bull. Soc. Math. France 144, 395 (2016), arxiv:1301.3042

[9] D. Calaque and M. Gonzalez, "On the universal twisted elliptic KZB connection", to appear.

[10] J. Broedel, N. Matthes and O. Schlotterer, "Relations between elliptic multiple zeta values and a special derivation algebra", J. Phys. A49, 155203 (2016), arxiv:1507.02254.

[11] N. Matthes, "Elliptic multiple zeta values", PhD thesis, Universität Hamburg, 2016.

[12] P. Lochak, N. Matthes and L. Schneps, "Elliptic multiple zeta values and the elliptic double shuffle relations", arxiv:1703.09410.

[13] G. Racinet, "Doubles mélanges des polylogarithmes multiples aux racines de l'unité", Publ. Math. Inst. Hautes Études Sci. 95, 185 (2002).

[14] J. Zhao, "Multiple polylogarithm values at roots of unity", C. R. Math. Acad. Sci. Paris 346, 1029 (2008)

[15] C. Glanois, "Periods of the motivic fundamental groupoid of $\mathbb{P}^{1} \backslash\left\{0, \mu_{N}, \infty\right\}$ ", PhD thesis, Université Pierre et Marie Curie, 2016.

[16] N. Matthes, "Elliptic double zeta values", J. Number Theory 171, 227 (2017), http://dx.doi.org/10.1016/j.jnt.2016.07.010

[17] K. Aomoto, "Special values of hyperlogarithms and linear difference schemes", Illinois J. Math. 34, 191 (1990).

[18] T. Terasoma, "Selberg Integrals and Multiple Zeta Values", Compositio Mathematica 133, 1 (2002), math/9908045

[19] F. Brown, "Multiple zeta values and periods of moduli spaces $\overline{\mathfrak{M}}_{0, n}$ ", Ann. Sci. Éc. Norm. Supér. (4) 42, 371 (2009), math/0606419.

[20] J. Broedel, O. Schlotterer and S. Stieberger, "Polylogarithms, Multiple Zeta Values and Superstring Amplitudes", Fortsch.Phys. 61, 812 (2013), arxiv:1304.7267.

[21] C. R. Mafra and O. Schlotterer, "Non-abelian Z-theory: Berends-Giele recursion for the $\alpha^{\prime}$-expansion of disk integrals", JHEP 1701, 031 (2017), arxiv:1609.07078

[22] O. Schlotterer and S. Stieberger, "Motivic Multiple Zeta Values and Superstring Amplitudes", J.Phys. A46, 475401 (2013), arxiv:1205.1516

[23] J. Broedel, O. Schlotterer, S. Stieberger and T. Terasoma, "All order $\alpha^{\prime}$-expansion of superstring trees from the Drinfeld associator", Phys.Rev. D89, 066014 (2014), arxiv:1304.7304.

[24] J. Drummond and E. Ragoucy, "Superstring amplitudes and the associator", JHEP 1308, 135 (2013), arxiv:1301.0794

[25] R. H. Boels, "On the field theory expansion of superstring five point amplitudes", Nucl. Phys. B876, 215 (2013), arxiv:1304.7918

[26] G. Puhlfuerst and S. Stieberger, "Differential Equations, Associators, and Recurrences for Amplitudes", Nucl. Phys. B902, 186 (2016), arxiv: 1507.01582 
[27] D. Oprisa and S. Stieberger, "Six gluon open superstring disk amplitude, multiple hypergeometric series and Euler-Zagier sums", hep-th/0509042.

[28] S. Stieberger and T. R. Taylor, "Multi-Gluon Scattering in Open Superstring Theory", Phys.Rev. D74, 126007 (2006), hep-th/0609175.

[29] S. Stieberger and T. R. Taylor, "Supersymmetry Relations and MHV Amplitudes in Superstring Theory", Nucl. Phys. B793, 83 (2008), arxiv:0708.0574.

[30] J. Broedel, C. R. Mafra, N. Matthes and O. Schlotterer, "Elliptic multiple zeta values and one-loop superstring amplitudes", JHEP 1507, 112 (2015), arxiv:1412.5535.

[31] S. Hohenegger and S. Stieberger, "Monodromy Relations in Higher-Loop String Amplitudes", Nucl. Phys. B925, 63 (2017), arxiv:1702.04963

[32] M. B. Green and J. H. Schwarz, "Supersymmetrical Dual String Theory. 3. Loops and Renormalization", Nucl.Phys. B198, 441 (1982).

[33] H. Tsunogai, "On some derivations of Lie algebras related to Galois representations", Publ. Res. Inst. Math. Sci. 31, 113 (1995).

[34] A. Pollack, "Relations between derivations arising from modular forms", Undergraduate thesis, Duke University.

[35] R. Hain and M. Matsumoto, "Universal Mixed Elliptic Motives", arxiv:1512.03975

[36] N. Matthes, "Decomposition of elliptic multiple zeta values and iterated Eisenstein integrals", arxiv:1703.09597, in: "Various aspects of multiple zeta value 2016", ed.: K. Ihara, Res. Inst. Math. Sci. (RIMS), Kyoto (2017), 170-183p.

[37] C. Reutenauer, "Free Lie Algebras", Clarendon Press Oxford University Press (1993).

[38] A. Weil, "Elliptic functions according to Eisenstein and Kronecker", Springer, Heidelberg, Published in "Ergebnisse der Mathematik und ihrer Grenzgebiete" (1976).

[39] D. Zagier, "Periods of modular forms and Jacobi theta functions", Invent. Math. 104, 449 (1991).

[40] V. Drinfeld, "On quasitriangular quasi-Hopf algebras and on a group that is closely connected with $\operatorname{Gal}(\overline{\mathbb{Q}} / \mathbb{Q})$ ”, Leningrad Math. J. 2 (4), 829 (1991).

[41] T. Le and J. Murakami, "Kontsevich's integral for the Kauffman polynomial", Nagoya Math J. 142, 93 (1996).

[42] B. Enriquez, "Quasi-reflection algebras and cyclotomic associators", Selecta Math. (N.S.) 13, 391 (2007), math/0408035.

[43] M. B. Green, J. Schwarz and E. Witten, "Superstring Theory. Vol. 1: Introduction", Cambridge, UK: Univ. Pr. (1987) (Cambridge Monographs on Mathematical Physics) (1987).

[44] M. B. Green, J. Schwarz and E. Witten, "Superstring Theory. Vol. 2: Loop amplitudes, anomalies and phenomenology", Cambridge, UK: Univ. Pr. (1987) (Cambridge Monographs on Mathematical Physics) (1987).

[45] J. Polchinski, "String theory. Vol. 1: An introduction to the bosonic string", Cambridge University Press (2007).

[46] J. Polchinski, "String theory. Vol. 2: Superstring theory and beyond", Cambridge University Press (2007).

[47] R. Blumenhagen, D. Luest and S. Theisen, "Basic concepts of string theory", Springer (2013), Heidelberg, Germany.

[48] R. Donagi and E. Witten, "Supermoduli Space Is Not Projected", Proc. Symp. Pure Math. 90, 19 (2015), arxiv:1304.7798, in: "Proceedings, String-Math 2012, Bonn, Germany, July 16-21, 2012", 19-72p.

[49] M. B. Green and J. H. Schwarz, "Infinity Cancellations in SO(32) Superstring Theory", Phys.Lett. B151, 21 (1985). 
[50] M. B. Green, J. H. Schwarz and L. Brink, "N=4 Yang-Mills and N=8 Supergravity as Limits of String Theories”, Nucl.Phys. B198, 474 (1982)

[51] C. P. Burgess and T. R. Morris, "Open and Unoriented Strings a La Polyakov", Nucl. Phys. B291, 256 (1987).

[52] C. P. Burgess and T. R. Morris, "Open Superstrings a La Polyakov", Nucl. Phys. B291, 285 (1987)

[53] P. Tourkine and P. Vanhove, "Higher-loop amplitude monodromy relations in string and gauge theory", Phys. Rev. Lett. 117, 211601 (2016), arxiv:1608.01665

[54] M. B. Green, J. G. Russo and P. Vanhove, "Low energy expansion of the four-particle genus-one amplitude in type II superstring theory", JHEP 0802, 020 (2008), arxiv:0801.0322.

[55] E. D'Hoker, M. B. Green and P. Vanhove, "On the modular structure of the genus-one Type II superstring low energy expansion", JHEP 1508, 041 (2015), arxiv:1502.06698.

[56] E. D’Hoker, M. B. Green, Ö. Gürdogan and P. Vanhove, "Modular Graph Functions", Commun. Num. Theor. Phys. 11, 165 (2017) arxiv:1512.06779

[57] "Digital Library of Mathematical Functions", http://dlmf.nist.gov.

[58] A. Tsuchiya, "More on One Loop Massless Amplitudes of Superstring Theories", Phys.Rev. D39, 1626 (1989).

[59] S. Stieberger and T. Taylor, "NonAbelian Born-Infeld action and type 1. - heterotic duality 2: Nonrenormalization theorems", Nucl.Phys. B648, 3 (2003), hep-th/0209064.

[60] N. Matthes, "On the algebraic structure of iterated integrals of quasimodular forms", Algebra Number Theory 11, 2113 (2017), arxiv:1708.04561.

[61] L. Kronecker, "Zur Theorie der elliptischen Funktionen", Mathematische Werke IV, 313 (1881).

[62] R. M. Hain, "The geometry of the mixed Hodge structure on the fundamental group", in: "Algebraic geometry, Bowdoin, 1985 (Brunswick, Maine, 1985)", Amer. Math. Soc., Providence, RI (1987), 247-282p.

[63] P. Deligne, "Le groupe fondamental unipotent motivique de $\mathbf{G}_{\mathbf{m}}-\mu_{N}$, pour $N=2,3,4,6$ ou 8 ", Publ. Math. Inst. Hautes Études Sci. 112, 101 (2010).

[64] J. Ablinger, J. Blumlein and C. Schneider, "Harmonic Sums and Polylogarithms Generated by Cyclotomic Polynomials", J. Math. Phys. 52, 102301 (2011), arxiv:1105.6063.

[65] D. Broadhurst, "Multiple Deligne values: a data mine with empirically tamed denominators", arxiv:1409.7204

[66] J. M. Henn, A. V. Smirnov and V. A. Smirnov, "Evaluating Multiple Polylogarithm Values at Sixth Roots of Unity up to Weight Six", Nucl. Phys. B919, 315 (2017), arxiv:1512.08389. 